\title{
Excessive appetite : the Pavlovian nature of human appetitive behaviour
}

Citation for published version (APA):

Havermans, R. C. (2005). Excessive appetite : the Pavlovian nature of human appetitive behaviour. [Doctoral Thesis, Maastricht University]. Datawyse / Universitaire Pers Maastricht. https://doi.org/10.26481/dis.20050311rh

Document status and date:

Published: 01/01/2005

DOI:

10.26481/dis.20050311rh

Document Version:

Publisher's PDF, also known as Version of record

\section{Please check the document version of this publication:}

- A submitted manuscript is the version of the article upon submission and before peer-review. There can be important differences between the submitted version and the official published version of record.

People interested in the research are advised to contact the author for the final version of the publication, or visit the DOI to the publisher's website.

- The final author version and the galley proof are versions of the publication after peer review.

- The final published version features the final layout of the paper including the volume, issue and page numbers.

Link to publication

\footnotetext{
General rights rights.

- You may freely distribute the URL identifying the publication in the public portal. please follow below link for the End User Agreement:

www.umlib.nl/taverne-license

Take down policy

If you believe that this document breaches copyright please contact us at:

repository@maastrichtuniversity.nl

providing details and we will investigate your claim.
}

Copyright and moral rights for the publications made accessible in the public portal are retained by the authors and/or other copyright owners and it is a condition of accessing publications that users recognise and abide by the legal requirements associated with these

- Users may download and print one copy of any publication from the public portal for the purpose of private study or research.

- You may not further distribute the material or use it for any profit-making activity or commercial gain

If the publication is distributed under the terms of Article $25 \mathrm{fa}$ of the Dutch Copyright Act, indicated by the "Taverne" license above, 


\section{EXCESSIVE APPETITE}

THE PAVLOVIAN NATURE OF HUMAN APPETITIVE BEHAVIOUR 
R.C. HAVERMANS, HELMOND 2005

DRUK: PONSEN \& LOOIEN BV, WAGENINGEN

Fot OMSLAG: INGE VAN GROESEN

ISBN 90-6464-846-8 


\section{EXCESSIVE APPETITE}

\section{THE PAVLOVIAN NATURE OF HUMAN APPETITIVE BEHAVIOUR}

PROEFSCHRIFT

ter verkrijging van de graad van doctor aan de Universiteit Maastricht, op gezag van de rector magnificus, prof. mr. G.P.M.F. Mols, volgens het besluit van het College van Decanen, in het openbaar te verdedigen op vrijdag 11 maart 2005 om 14:00 uur door

REMCO CORNELIS HAVERMANS 
PRomotor:

Prof, dr. A.T.M. Jansen

BEOORDRLINGSCOMMISSIE:

Prof. dr. HL.G. Merckellbach (voorzitter)

Prof. dr. H.F.M. Crombag

Prof. dr. M.A. van den Hout (Universiteit Utrecht)

Dr. J.H.R. Maes (Radboud Universiteit Nijmegen)

Dr. R.W. Wiers 


\section{CONTENTS}

1 GENERAL INTRODUCTION

2. The Pavlovian Nature of an Excessive Appetite

4 The Pavlovian Nature of an Excessive Appetite for Drugs and Alcohol

$8 \quad$ The Pavlovian Nature of an Excessive Appetite for Food

$10 \quad$ Outline of the Present Thesis

13 CHAPTER 1. Blocking and Unblocking of Causal Judgment: The Role of Outcome Magnitude on Human Causal Learning

$16 \quad$ Experiment 1

Method

18

Results and Discussion

19

20

Experimen 2

21

23

24

Method

Results and Disicussion

Experiment 3

Merhod

25

26

Results and Disicussion

General Discussion

\section{PART I. The Pavlovian Nature of an Excessive Appetite for Drugs and Alcohol}

31 CHAPTER 2. The Efficacy of Cue Exposure with Response Prevention in Extinguishing Drug and Alcohol Cue Reactivity

Method

Results

Discussion

43. CHAPTER 3. The Effect of Cue Exposure, Urge to Smoke, and Nicotine Deprivation on Cognitive Performance in Smokers

$46 \quad$ Method

$49 \quad$ Results

54 Discussion 
57 CHAPTER 4. Increasing the Efficacy of Cue Exposure Treatment in Preventing Relapse of Addictive Behaviour

59 Theoretical Notess

61 Conclusion

63 CHAPTER 5. Contextual Control of Extinguished Conditioned Performance in Humans

66

Experiment I

Method

69

Results and Discussion

71

Experiment 2

Method

72

Results and Discussion

75

Experiment 3

Method

76

Results and Discussion

80

General Discussion

83 CHAPTER 6. Context Dependent Access to Alcohol-Related Concepts Stored in Memory

85

Method

86 Results

88 Discussion

\section{PART II. The Pavlovian Nature of an Excessive Appetite for Food}

93 CHAPTER 7. Obese Women Demonstrate Stronger Sensory-Specific Satiety than Normal-Weight Women

95 Method

97 Results

102 Discussion

105 CHAPTER 8. Comparing Carbohydrates and Dietary Fat in Conditioning Flavour Preferences in Humans

107 Method

109 Results

111 Discussion 
113 CHAPTER 9. Stimulus Generalisation and Configural Learning as a Model of Evaluative Conditioning

The associative natwe of evaluative conditioning Models of EC

139 SUMMARY

145 SAMENVATTING

151 REFERENCES

163 DANKWOORD

165 CURRICULUM VITAE

167 PLBLICATIES 



\section{GENERAL INTRODUCTION}

I went on a diet, swore off drinking and heavy eating, and in fourteen days I lost two weeks.

Joe E. Lewis 


\section{The Pavlovian Nature of an Excessive Appetite}

The central theme of the present thesis concerns studies investigating the learned nature of human appetitive behaviour gone out of control. Such behaviour is characterised by an excessive appetite with its primary examples being obesity and addiction (see Orford, 2001 ). A drug addict displays an excessive appetite (or craving) for drugs, whereas the obese person displays an excessive appetite for particularly food high in fat content (Rissanen, et al., 2002).

There are several reasons for specifically investigating the role of learning in excessive appetitive behaviour. The frrst is keen interest underlying all research. The second reason is that studies on learning have provided important insights into how experience changes, or shapes behaviour, and thus may also provide insight into how an excessive appetite is acquired. Such learning of behaviour can be defined as "a relatively permanent change in the probability of exhibiting a certain behaviour resulting from some prior experience" (Mowrer \& Klein, 2000, p. 2). One of the most basic forms of learning through experience is Pavlovian conditioning, associating a particular stimulus with a specific outcome. For example, in one of Pavlow's (1927) experiments with a dog, the intake of food by the dog (i.e., the outcome) was preceded by the sound of a metronome. Pavlov (p. 26) noted that "after several repetitions of the combined stimulation, the sounds of the metronome had acquired the property of stimulating salivary secretion". Pavlov described this conditioning of a salivary reflex as resulting from the establishment of a link, or association, between the presentation of a biologically neutral conditioned stimulus (CS; e.g., the sound of a metronome) and the biollogically relevant unconditioned stimulus (US) food. In other words, the dog had learned that the metronome predicts or causes food intake and thus the dog came to salivate to the sound of the metronome in the anticipation of food.

Pavlov's research on conditioning sparked the interest of many psychologists, and it still cloes today. Pavlov himself, though, never aspired to be of any relevance for psychological science. As a physiologist, he strongly believed that his conditioning experiments would lay the foundation for understanding the workings of the cerebral hemispheres. Surely the problem of understanding brain functioning could not be left into the hands of psychologists: "if we attempt an approach from this science of psychology to the problem confronting us we shall be building our superstructure on a science which has no claim to exactness as compared even with physiology. In fact it is still open to discussion whether psychology is a natural science, or whether it can be regarded as a science at all." (Pavlow, 1927, p. 3). Despite Pavlov's reservations about psychological science, psychologists such as Yerkes and Skinner readily recognised the importance of Pavlov's work in turning psychology into a true science of behaviour (see Yerkes \& Morgulis, 1909; Catania \& Laties, 1999). This notion contributed to a body of research now covering almost a century of both animal and human studies that is generally referred to as learning theory. Initially, learning 
theory research was primarily aimed at testing the generality of conditioning. Indeed, associative learning has been found to be a very general phenomenon. Pavlovian conditioning phenomena, for example, could be demonstrated in a wide variety of species, ranging from honey bees to humans (Branch \& Hackenberg, 1998). Further, it was found that Pavlovian conditioning is not restricted to reflexive behaviour. Psychophysiological and overt behavioural responses, motivational states, and expectations can all be conditioned (Lieberman, 1993).

A third reason for studying the associatively learned nature of human behaviour is the historic relevance of learning theoretical research for the development of behavioural methods to treat human psychopathology. Applying the principles of Pavlovian conditioning in clinical research and practice has proven to be extremely beneficial for both understanding and treating psychopathology. This led to the development of diverse behavioural interventions that - mostly as part of a cognitive behavioural treatment program - have become the treatment of choice in most psychopathologies (O'Donohue, 1998). At the same time, both clinical researchers and therapists have gradually lost touch with contemporary learning theory (O'Donohue, 1998). It is not entirely clear why this is so, but one reason may be that learning theory research mainly comprises animal studies. Despite the generality of conditioning phenomena and although animal experiments have been useful in the development of therapeutic applications, both fundamental and clinical researchers agree that there exists a huge gap between the behaviour of a laboratory rat and a patient. The gap could be bridged - in part - by fundamental research on how associative learning processes determine human learning and behaviour (Matute \& Miller, 1998). Humans are animals, but of course not all animals are necessarily human. People often do, but sometimes do not behave like a lab rat (see Branch \& Hackenberg, 1998). What is clear is that the waning interest in contemporary learning theory research is unfortunate as learning theory is still a dynamic research area generating novel findings that can be particularly relevant for clinical practice.

A fourth reason is that it is clear that one is not simply born with an excessive fat preference or an intense craving for drugs or alcohol. Of course, one may be genetically predisposed to develop a drug addiction or to become obese, but experience with substances and food is a prerequisite for the acquisition of an excessive appetite for drugs or high fat food. In other words, excessive appetites have an important learned component. Understanding how learning processes contribute to the development of an excessive appetite may provide important insights for developing or improving treatment techniques. This is important as both addiction and obesity disrupt and shorten lives, and have proven to be extremely resistant to treatment (Orford, 2001; Lowe, 2003; McLellan, Lewis, O'Brien, \& Kleber, 2000).

The present thesis thus concerns the learned nature of an excessive appetite; that is, an excessive appetite for drugs and alcohol (part I of the present thesis), or an excessive 
appetite for food (part 11). The following sections provide a theoretical background and a short introduction to the research questions addressed in the subsequent chapters of this thesis.

\section{The Pavlovian Nature of an Excessive Appetite for Drugs and Al- cohol}

Addiction can be regarded as appetitive behaviour gone out of control. Learning is generally considered to play an important role in the development of an addiction. For example, because of the rewarding properties of psychotropic substances, drug use is positively reinforced leading to an increase in the strength and frequency of drug use (see Heather \& Robertson, 1997). According to the Law of Effect any behaviour that has positive consequences should increase in frequency and strength and should decrease when it has negative consequences (Lieberman, 1993). This operant conditioning view, however, has received considerable criticisms. Heyman (1996), for instance, argues that drug use not only has positive consequences, but as drug use becomes more frequent it also has negative consequences that increase in intensity and duration. Furthermore, due to the development of drug tolerance the rewarding effect of drug use becomes increasingly smaller with prolonged drug use. In other words, the Law of Effect predicts that drug use should eventually decrease in strength and frequency. This does not appear to be the case. Addiction is characterised by an excessive appetite for drugs, or craving, despite the adverse effects of drug use and drug tolerance. The development of such craving cannot be explained in terms of operant conditioning.

Craving is not a tonic state. Episodes of intense craving are primarily experienced when axposed to cues predicting drug intake. Further, the strength of such cue-elicited craving has been found to correlate with the amount of experience with drug consumption. This clearly suggests that craving can be conceptualised as a Pavlovian conditioned response (see Drummond, Tiffany, Glautier, \& Remington, 1995).

The suggestion that Pavlovian conditioning may play an important role in the etiology of addiction is not a novel idea. Pavlov (1927) himself has described the work of doctor Krylov in quite some detail, providing the very first demonstration of the potential role of conditioning processes in the etiology of addictive behaviour. Krylov, at that time working at the Tashkent Bacteriological Laboratory, had found that the initial response to the injection of morphine in dogs (i.e., mausea, salivary secretion, and subsequent sleep), could be demonstrated in dogs repeatedly administered morphine on previous occasions after merely experiencing the preparatory manipulations preceding actual morphine injection. 
Krylov was able to replicate this effect in Pawlov's laboratory at the Institute of Experimental Medicine in St. Petersburg. Pavlov (p. 36) notes:

The dog has repeatedly been injected with morphine on previous occasions, and is now held quietly on the table by an attendant who has never had anything to do with injecting the morphine. When the experimenter approaches, the dog gets restless and moistens its lips, and as soon as the experimenter touches the animal, severe nausea and profuse secretion of saliva begin.

Since Pavlov, three major associative learning models of addiction have been conceptualised: the conditioned withdrawal model, the conditioned compensatory drug response model, and the conditioned incentive motivation model. Abraham Wikler was the first psychologist to explicitly suggest that Pavlovian conditioning processes play an im portant role in the development, maintenance and relapse of addictive behaviour. According to Wikler (1948), repeated drug use leads to the formation of an association between drug related cues and the aversive withdrawal effects experienced after prolonged and repeated drug use. As such, these drug cues come to elicit conditioned withdrawal responding. This is experienced as a strong urge to use drugs in order to alleviate the sudden aversive state of withdrawal. Although it does provide a quite compelling wiew on the maintenance of addictive behaviour, there is not much empirical evidence in support of the notion that drug cues specifically lead to the experience of drug withdrawal effects (see Glautier \& Remington, 1995).

Siegel (1983) proposed a somewhat different associative account of addictive behaviour. According to Siegel, repeated drug use leads to the formation of an association between drug-related cues and the effect of a given drug. When one is exposed to such a cue, this elicits a conditioned preparatory, physiologicall response in anticipation of the upcoming intake of a psychoactive substance. More specifically, the body reacts opposite to the effects of the drug hence compensating for the disruptive effect of the drug on homeostatic functioning. This compensatory responding is usually experienced as an aversive state, and this is subsequently experienced and expressed as a strong urge to use drugs. One primary prediction of this view is that one should display situation-specific tolerance to drugs, because compensatory drug responding is expected within the drug use emvironment, but not outside this situation (i.e., in the absence of drug-rellated cues). Such conditioned tolerance has indeed been demonstrated in both animal and human subjects (see Siegel \& Ramos, 2002). However, this model has been criticised as well. In some in-

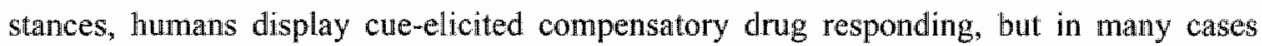
psychophysiological responding to drug cues appears to be isodirectional to the effect of the drug (Stewart, de Wit, \& Eikelboom, 1984). Stewart and colleagues argue that it is the conditioned isodirectional drug response that leads to an appeal on the incentive motivation 
for drugs and is experienced as strong urges. Indeed, several studies have demonstrated cue-elicited psychophysiological responding to be isodirectional to the effect of the drug (see Glautier \& Remington, 1995).

The debate concerning the directionality of psychophysiological responding to drug cues is still unresolved. This, however, does not imply that addictive behaviour cannot be described in terms of associative learning. Despite their differences, these associative learning models of addictive behaviour regard cue-elicited craving as the subjective manifestation of conditioned drug responding, or cue reactivity. Furthermore, according to these models such cue reactivity forms the primary motivation for the maintenance of addictive bebaviour and relapse. Carter and Tiffany (1999) however, have argued that urges do not necessarily reflect drug-specific conditioned psychophysiological responding. In their meta-analysis, they found that the psychophysiological response patterns to drug cues are remarkably similar across different types of drugs and that this particular response pattern does not correlate very highly with self-reported urges. They conclude that psychophysiological responding to drug cues may represent a more general arousal response, rather than the physiological instantiation of subjectively experienced urges. However, such a general arousal response can still be argued to reflect the associatively acquired significance of drug cues, and can thus still be regarded as a physiological measure of cue reactivity. If so, then it might be possible to extinguish such responding. Namely, Pavlov (1927) not only demonstrated that responses could be conditioned, but also that these conditioned reflexes could subsequently be extinguished due to repeated nonreinforced presentation of the CS. This extinction procedure provides the theoretical basis for cue exposure with response prevention therapy. In this type of behavioural treatment, the patient is repeatedly exposed to drug related cues, but prevented from subsequent drug use. Gradually, the cues lose their predictive value regarding the occurrence of a drug effect, hence leading to the extinction of cue reactivity. In previous studies it has been demonstrated that the subjective urge to use drugs can be successfully extinguished, but very few studies have investigated whether this is also true for cue-elicited psychophysiological responding (see Drummond, et al., 1995). Whether cue exposure with response prevention effectively extinguishes both subjective and psychophysiological cue reactivity was investigated in chapter 2.

Initially, with the demonstration of successful extinction of drug urges, cue exposure with response prevention was regarded as a promising therapeutic tool to diminish the probability of relapse (Heather \& Bradley, 1990). Relapse poses the biggest problem in the treatment of addiction. Most patients treated for substance dependence will relapse within a year after treatment (see e.g., Hunt, Barnett, \& Branch, 1971; McLellan, et al., 2000 ). Surely, with the elimination of one of the primary motivations for continued drug use, the probability of a relapse should be limited (Heather \& Bradley, 1990). However, there is no evidence that cue exposure with response prevention limits the probability of 
relapse in addictive behaviour (Conklin \& Tiffany, 2002), At best, cue exposure therapy appears to prolong the period of abstinence after treatment (see Drummond \& Glautier, 1994).

The limited effect of cue exposure therapy puzzled climical researchers, but it could have been foreseen. The rationale of cue exposure therapy is based on the assumption that extinction of urges reflect the unlearning of the underlying association between cues and drug intake. Heather and Robertson (1997), for example, assume extinction of the urge to drink alcohol to be the result of breaking the learned link between alcohol-related cues and drinking. This assumption, however, is false. Pavlov (1927) already stated that "Left to themselves extinguished conditioned reflexes spontaneously recover their full strength" ( $\mathrm{p}$. 58). Therefore, Pavlow (p. 60) concludes that "extinction must be regarded as a special form of inhibition. That it cannot be regarded as an irreparable destruction of the conditioned reflex. . is evidenced by the fact that the extinguished reflexes spontaneously regenerate in course of time." Bouton (1993) also explains such renewal of conditioned responding by arguing that during extinction the CS acquires an additional inhibitory meaning. Af ter extinction then, the CS predicts both the occurrence and the absence of the US. This ambiguous meaning of the $\mathrm{CS}$ is disambiguated by contextual information, that is, the inhibitory meaning is only retrieved in the temporal and environmental context in which extinction has taken place. Outside this particular context the excitatory meaning of the CS is retrieved. The implications of renewal of extinguished conditioned responding for the applicability of cue exposure with response prevention in clinical practice are discussed in chapter 4 . The mosit relevant implication is that the renewal effect implies that outside the clinical treatment setting, cues can still elicit conditioned drug responding, rendering cue exposure therapy in clinical settings ineffective as a means to prevent relapse (Conklin \& Tiffany, 2002). This implication, however, is solely based on animal research. The question that arises then is whether extinguished conditioned responses are equally context specific in human subjects. This question is addressed in chapter 5 .

More recently, associative models of addiction have been challenged by more cog. nitive models of addictive behaviour. One of the most prominent of these models concerns the automatic drug use and drug urge model by Stephen Tiffany (1990). According to Tiffany, frequent drug use leads to the fotmation of a drug use action schema comprising all behavioural elements of addictive behaviour. This action schema allows for the automatic and almost reflexive use of drugs, requiring little if any conscious cognitive processing. As long as such automatic drug use can be executed, urges do not play an important role in the maintenance of this behawiour. If on the other hand the execution of the action schema is blocked, capacity-limited cognitive resources are addressed to ascertain drug use. This is experienced as urges or craving. The urge to use a given drug should thus reflect the operation of cognitive processes at the expense of other tasks requiring the same limited re- 
sources (Tiffany, 1990). Whether this is indeed the case was investigated in smokers, results of which are described in chapter 3 .

Another prominemt cognitive model of addictive behaviour is the memory network model. According to this model, frequent drug use leads to the formation of a drug-related mentioty network comprising interlinked nodes representing different drug-related concepts or cognitions. The more one uses drugs, the more ellaborate this network becomes, the more thoroughly interconnected this network becomes and hence the more accessible (see Wiers, et all.,2002). Activation of this memory network through priming has been found to predict drug or alcohol use (Stacy, 1997). Glautier and Spencer (1999) have pointed to the conceptual overlap between the memory network model and associative models of addictive behaviour; in the sense that both models generally assume that repeated drug use leads to the formation of drug-related associations represented in memory. However, according to the associative learning view, drug-related cues should be able to elicit, or activate these associations represented in memory. Glautier and Spencer showed that the accessibility of an alcohol memory network is indeed influenced by the presence or absence of alcoholrelated cues. Participants generated more alcohol-related cognitions after being exposed to an alcoholic beverage. As drinking of alcohol has been found to be situation specific, this priming effect of alcohol cues may be influenced by the context (i.e., an environment that is drinking-related or not) in which these are presented. The role of context on the activation of alcohol related cognitions stored in memory was investigated in chapter 6.

\section{The Pavlovian Nature of an Excessive Appetite for Food}

Preferences and aversions, or likes and dislikes, are important determinants of human behaviour. Some of these likes and dislikes can be innate, such as the imnate preference for sweet tastes and the innate aversion for bitter tastes (see Capaldi, 1996). However, most of our preferences and aversions are acquired with experience. Associative learning processes have often been proposed as the primary mechanism underlying the development of such likes and dislikes.

Experience with food can change the liking of that particular food. For example, after a food or drink has been consumed to satiety, the palatability of that food will have decreased markedly as compared to other foods uneaten. This effect has been termed sensory-specific satiety (Rolls, 1986), and plays an important role in meal termination. As the decrease in palatability already occurs during consumption and hence does not depend on any postingestive signals, th has been suggested that sensory-specific satiety is the result of habituation to the sight, smell and taste of the food (Swithers \& Hall, 1994; Hetherington \& Rolls, 1996). It has been suggested that obesity is the result of obese persons demon- 
strating less sensory-specific satiety than normal-weight persons. As meal termination is at least partly determined by sensory-specific satiety, demonstrating less such an effect would promote overeating predisposing one for developing overweight and subsequent obesity (see Raynor \& Epstein, 2001). This hypothesis was tested in chapter 7 .

Individual differences in sensory-specific satiety can explain why obese persons tend to overeat, but it fails to account for the apparent fat preference that obese persons typically display (see Rissanen, et al. 2002). It has been argued that the acquisition of such taste preferences are best understood in terms of Pavlovian conditioning (Fedorchak, 1997; Capaldi, 1996).

Pavlov (1927) himself noted that his dogs could differentially display a conditioned salivary reflex dependent upon the content of the US. An aversive US (e.g., an ellectric shock) would elicit a defensive salivary reflex, poor in mucin, whereas appetitive USs elicited an appetitive mucin-rich salivary reaction. Pavlow, however, did not explain this in terms of a hedonic response system. Such a hedonic view on the acquisition of likes and dislikes was not formulated until the late $60^{\prime} \mathrm{s}$ of the twentieth century after the work on conditioned taste aversion by Garcia and Koelling (1966). Taste aversion learning entails giving an animal a flavoured solution to consume and then making the animal feel sick by, for example, injecting the animal with a dose of lithium-chloride. The animal will associate the taste (CS) of the solution with becoming ill (US) and hence will suppress any further ingestion of that taste. Robert Bolles was fascinated by this line of research and started studying conditioned taste aversions and preferences as well (see Fedorchak, 1997). Conditioned taste preferences can be acquired by consequently pairing a neutral taste (CS) with a caloric reinforcer (US: e.g., nutrients such as sugar, fat, or alcohol). Such conditioning of taste preferences was thus termed "caloric conditioning". Mehiel and Bolles (1988) found evidence that not the total amount of calories provided by the test food, but the caloric density of the food determined the speed of the acquisition of a conditioned taste preference in rats. Such conditioning of taste preferences has also been demonstrated in humans. In several studies it has been shown that children will come to display a conditioned preference for flavours when these flavours are paired with a bigh dietary fat content (lohnson, McPhee, \& Birch, 1991; Kern, McPhee, Johnson, Fisher, \& Birch, 1993). More recently, Sclafani (1997) has contested that caloric density alone determines conditioning of taste preferences. Sclafani found that different nutrients can differ in their efficacy to condition a taste preference, regardless of their caloric density. More specifically, carbohydrates (e.g., sugar) appear to lead to a much more rapid acquisition of conditioned taste preferences than isocaloric dietary fat in rats (Lucas \& Sclafani, 1999a). It thus appears that different. nutrients have their own specific postingestive effects, apart from providing energy, which can affect the acquisition of a taste preference. This hypothesis was tested in human subjects, results of which are described in chapter 8 . 
Another way to acquire taste preferences is through flavour-flavour learning; pairing a neutral flavour with an already preferred flavour (e.g., the sweet taste of saccharine). This flavour-flavour learning too has been demonstrated in both animals (Fedorchak, 1997; Capaldi, 1996) and humans (see e.g., Zelher, Rozin, Aron \& Kulish, 1983). Apparently, some of the positive hedonic value of a sweet taste can transfer to the neutral taste. This kind of evaluative conditioning is not restricted to flavours, it has also been found within the visual, tactile, and auditory domain (see De Houwer, Thomals \& Baeyens, 2001). Capaldi (1996) noted, though, that it is difficult to conceptualise such evaluative conditioning in terms of CSs and USs, as a US in Pavlovian conditioning usually is a biologically relevant stimulus, whereas in evaluative conditioning the US is not necessarily such a biologically relevant stimulus. It is, therefore, unclear whether evaluative conditioning reflects Pavlovian conditioning, or even whether such evaluative learning reflects associative learning at all (see De Houwer, et al., 2001). In chapter 9, it is argued that evaluative conditioning does not reflect associative learning, but that the transfer of hedonic-affective value is best described in terms of stimulus generalisation.

\section{Outline of the Present Thesis}

The present thesis represents studies concerning the Pavlovian nature of an excessive appetite for drugs and alcohol (part I) and the learned nature of an excessive appetite for food (part II). More specifically, the research questions addressed in the following chapters are: (i) can cue-elicited drug craving and psychophysiological responding be extinguished? (chapters 2,4 and 5), (ii) what is the role of environmental context in the expression and extinction of cue reactivity? (chapters 4,5 , and 6 ), (iii) does the urge to smoke reflect the operation of nonautomatic cognitive processes? (chapter 3), (iv) how may sensory-specific satiety contribute to the development of obesity (chapter 7)?, (v) do different macronutrients have differential reinforcing potencies in the conditioning of a flavour preference in humans? (chapter 8), and (vi) to what degree can the acquisition of likes and dislikes be ascribed to associative learning processes (chapter 9)?

These chapters are preceded by a study concerning the Pavlovian nature of human causal learning (chapter 1). Contemporary learning theorists often refer to Pavlovian conditioning as being causal learning; learning that one event causes a certain outcome (Dickinson, Shanks, \& Evenden, 1984; Rescorla, 1988). Dickinson and colleagues even stated that the reverse may also be true, that is, causal learning reflects associative learning (see also Allan, 1993). Indeed, associative learning can be described as causal learning, but this does not logically imply that all causal inference is based on associative learning. Nonetheless, it is an interesting and testable hypothesis that generates two specific predictions. Firstly, it 
should be possible to replicate well-described conditioning phenomena in humans using a causal learning paradigm. Secondly, causal inference should depend on learning through experience. In a series of three experiments, these specific predictions were tested, the results of which are presented in chapter 1. As argued above, such fundamental research on how associative learning processes determine human learning and behaviour contributes to bridging the gap between learning theory research and clinical practice. 


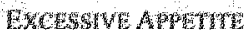




\title{
CHAPTER 1
}

\section{BLOCKING AND UNBLOCKING OF CAUSAL JUDGMENT: THE ROLE OF OUTCOME MAGNTUDE ON HUMAN CAUSAL LEARNING*}

\begin{abstract}
Blocking of causal judgment refers to the phenomenon in which the learning of a cansal relation between at cue $\mathrm{X}$ and a certan outcome is attenuated when this cte is presented in connpound with another cue $A$ that already serves as a strong predictor of the outcorne. It has been suggested that this phenomenon indicates that human causal learning reflects associative learning processes. According to this perspective, unblocking of causal judgment of $X$ should ocew when on AX-outcome trialls the outcome magnitude exceeds the outcome magnitude on the preceding A-outcome trials. In a series of three experiments it was investigated whether such a manipulation leads to unblocking of causal judgment. Such unblocking was found, but did not depend on trial order, or on trial-by-trial presentation of cue-outcome contingencies. It is ar gued that these results prowide support for the view that human causal inference depends on more general deductive reasoning processes, rather than on associative learning alone.
\end{abstract}

\footnotetext{
- This chapter is an adapted version of: Havermans, R.C., Vancleef, L. \& Jansen, A. (2004). Blocking and whblocking of causal judgment: The role of outcome magnitude on human causal hearning Manuscript submitted for publication.
} 
In the last wo decades, human causal (or contingency) learning has received considerable interest. This interest was fuelled mainly by the suggestion made by several investigators that human causal learning inwolves Pawlovian conditioning processes (see e.g., Allan, 1993; De Howwer \& Beckers, 2002; Dickinson, Shanks, \& Evenden, 1984). In Pavlovian conditioning; a conditioned stimulus (CS, e.g., a tone) is repeatedly paired with a given outcome (e.g., an electric shock). As such, the CS becomes endowed with a signalling function, that is, the CS comes to signal or predict the outcome. In most human causal learning tasks, a described cue (e.g.n a food) is repeatedly paired with a certain described outcome (e.g., an allergic reaction). As a consequence participants will come to judge this particular cue as the cause of the outcome. If one assumes that such causality detection reflects associative learning, then it should be possible to replicate animal conditioning phenomena in humans employing causal llearning tasks. One such phenomenon concerns blocking.

Effective conditioning requires that the $\mathrm{CS}$ is a nonredundant predictor of the outcome. Kamin (1969) demonstrated this in rat subjects. Rats fail to learn an association between a target stimulus $X$ and an outcome, when $X$ is presented in compound with a stimulus $A$ that has alleady been established as a sufficient predictor of the outcome on previous trials. The A-outcome association is said to block the acquisition of an X-outcome association. Such blocking has frequently been demonstrated in the context of human causal learning (see e.g., Chapman, 1991; Chapman \& Robbins, 1990; Dickinson, et al., 1984). The Rescorla-Wagner (RW) model (Rescorla \& Wagner, 1972) of associative learning readily accounts for such blocking of causal judgment. According to the RW model, a change in associative strength $V$ of a cue $\mathrm{X}$ on a given trial is described by

$$
\Delta V_{X}=\alpha_{X} \beta_{O}\left(\lambda-\sum V\right)
$$

where $\alpha_{X}$ and $\beta_{o}$ retlect the salience of $\mathrm{X}$ and the outcome $\mathrm{O}$ respectively, the parameter $\lambda$ refers to the maximum level of associative strength that the outcome can sustain and $\sum V$ is the total of associative strength of all present cues. As associative strength of $X$ increases, the error-term $\left(\lambda-\sum V\right)$ will gradually approach a value of zero, leading to a typical negatively accelerated learning curve. In the case of blocking, on $A X-O$ compound trials the error-term will be close to zero as cue $A$ has already gained maximum associative strength in the preceding A-O trials, hence little if any learning is expected to target cue $\mathrm{X}$.

The RW model however, is not the only model of causal learning that can account for blocking of causal judgment. Statistical models of human causal learning, such as the probabilistic contrast model (Cheng \& Novick, 1992) and the power PC model (Cheng, 1997), also provide an adequate explanation of such blocking effects. For example, according to the probabilistic contrast model, causal judgment of target cue $\mathrm{X}$ in a blocking para- 
digm is determined by the intuitive calculation of the contrast between the probability that the outcome occurs in the presence of cue $\mathrm{A}$ and $\mathrm{X}(P[\mathrm{O} \mid \mathrm{AX}])$ and the probability of the outcome in the presence of $A$ alone $(P[O \mid A \sim X])$. However, these statistical models of causality detection only apply to the detection of causality between cues and a dichotomous outcome (i.e., outcome present, or absent) (see De Houwer, Beckers, \& Glautier, 2002). In real-world situations outcomes may vary in magnitude (e.g., smoking causes health problems, but the seriousness of these health problems may vary over time within an individual and may differ between individuals). According to the $\mathrm{RW}$ model, variations in ontcome magnitude affect the parameter $\lambda$, that is, the larger the outcome the more associative strength the outcome can sustain. Therefore, the RW model predicts stronger causal judgments with larger outcomes. Indeed, this is exactly what Cramer et al. (2002) found. In one of their experiments, participants had to estimate the strength of a causal relation between a worker $\mathrm{X}$ and production. Production levels were varied, ranging from low to medium to high. The group of participants who had observed that worker $X$ covaried with high pro. duction levels, estimated the strength of the causal relation between worker $X$ and production much stronger than participants that had observed the contingency $X$-Medium production level. Similarly, participants who observed that worker $X$ covaried with a medium production level judged causal relation between worker $X$ and production stronger than participants who had observed that worker $X$ correlated with a low production level.

The RW model also specifically predicts that no blocking, or unblocking, should occur to a target cue $\mathrm{X}$ when on the compound $\mathrm{AX}-\mathrm{O}$ trials the outcome magnitude is larger than the magnitude of the outcome on the preceding A-O trials. In this case, the errorterm at the start of the compound trials will be larger than zero allowing for the learning of a causal relation between $\mathrm{X}$ and the outcome (see also Lovibond, Been, Mitchell, Bouton, \& Frohardt, 2003). Indeed, such unblocking due to variations in outcome magnitude has already been successfully demonstrated in animals (see Kamin, 1969).

The primary aim of the present study was to investigate whether unblocking of causal judgment to a target cue $X$ occurs as the result of presenting a larger outcone on the compound $\mathrm{AX}-\mathrm{O}$ trials. This was tested in a series of three experiments in which participants had to assume the role of a medical researcher determining whether exposure to certain fictional chemical substances causes the development of a fictional disease called Miltosis. 


\section{Experiment 1}

\section{Method}

Paricipants. A total of 32 undergraduate students (2l women; 11 men) of the Maastricht Uniwersity took part in the present experiment $(M$ age $=19.8$ years, $S D=1.8)$. They received course credits upon completion of the experiment.

Apparatus. All participants were tested individually in a quiet research room at the Maastricht University. The causal learning task was presented on an IBM-compatible personal computer and was programmed in Experimental Runtime System (ERTS, Berisoft, Germany).

Procedure and Design. A local ethical committee approved the experiment. Participants were randomly assigned to one of two groups of equal size $(n=16)$ : group Blocking, or Control. On arrival the participant was informed about the general procedure of the experiment and was told that the main purpose of the experiment was to determine how people detect causal relations. After obtaining informed consent, the participant was seated at a computer and requested to follow the instructions on the screen. On two consecutive screens the following instructions were displayed (translated from Dutch):

(1st screen) Imagine you"re a medical researcher and that recently you discovered a novel disease you termed Miltosis. This disease comprises five distinct symptoms and may vary in intensity, that is, the more symptoms the more serious the disease. Although the disease is rare, you have observed that the incidence of the disease is relatively high among workers in the chemical industry. Therefore, you hypothesise that exposure to certain chemicals causes the occurrence of Miltosis. To investigate this hypothesis, you have tested several workers who may be exposed to dangerous chemical substances in their work. You determined to which chemical substances they are exposed to churing their work and whether they develop any symptoms of Miltosis over time. (2nd screen) You will be shown the research results of all investigated persons, that is, you will be shown the substance(s) to which these persons have been exposed. For each person $X$ you need to judge to what degree you believe they will show symptoms of Miltosis on a rating scale ranging from -100 (absolutely certain of NO symptons of Miltosis) to 0 (no idea) to + 100 (absolutely certain of symptoms of Miltosis). Alter providing your judgment, you will be shown how many symptoms this person has developed.

After these instructions participants in the Blocking group were first shown 8 trials in which exposure to a substance A was always followed by 3 symptoms of Miltosis, 8 trials in which exposure to a substance B was also always followed by 3 symptoms of Miltosis, and 8 trials in which exposure to a substance $C$ never led to the development of any symptoms. Trials were presented ir a random order. For each presentation of a research lile, participants indicated to what degree they believed that the given person $X$ has developed symptoms of Miltosis. They could do so on a $100 \mathrm{~nm}$ Visual Analog Scale (VAS) displayed on screen ranging from -100 at the extreme left (absolutely certain of NO symptoms of Miltosis) to 0 in the middle (no idea) to 100 (absolutely 
certain of symptoms of Miltosis). They could use the computer nouse to drag a pointer anywhere on the scale, and by clicking on a given position on the scale they could make their prediction. Immediately after making their prediction, a $3 \mathrm{sec}$ feedback message was shown at the center of the screen stating how many symptoms of Miltosis the given person has developed.

The second learning phase was equal for both groups. In this phase, all participants received the presentation of 8 compound trials in which a compound comprising exposure to both $\mathrm{A}$ and a substance $X$ was followed by 5 symptoms of Miltosis, whereas the compound $B$ and a substance $Y$ was followed by 3 symptoms of Miltosis. In addition, 8 trials were presented in which exposure to substance $\mathrm{C}$ was never followed by the development of symptoms. This phase immediately followed the preceding learning phase for the participants in the Blocking group and trials were once again presented in a completely random order for each separate participant.

At test, participants had to indicate for each separate substance to what degree they believe that exposure to that substance causes the development of symptoms of Miltosis on the same type of rating scale as during the previous learning phase(s). During the entire experinnent different fictional substance names served as cues $A, B, C, X$, and $Y$, namely: Nafteen, Tramine, Morphide, Glexus and Phenidaat. The names were assigned randomly to function as cue $A, B, C, X$, or $X$. The experimental design is summarised in Table 1.1.

Table 1.1

Design of experiment 1.

\begin{tabular}{|c|c|c|c|}
\hline & \multirow[b]{2}{*}{ One } & hase & \multirow[b]{2}{*}{ Test } \\
\hline & & Two & \\
\hline & $A-3$ & $A X=5$ & A? \\
\hline Group & $B-3$ & $B Y-3$ & $\mathbb{B}$ ? \\
\hline \multirow[t]{3}{*}{ Blocking } & $C-0$ & $C-0$ & $C ?$ \\
\hline & & & $x ?$ \\
\hline & & & $Y ?$ \\
\hline & & $A X=5$ & $\overline{A ?}$ \\
\hline Group & & $B Y-3$ & $B ?$ \\
\hline \multirow[t]{3}{*}{ Control } & & $C-0$ & $\mathrm{C?}$ \\
\hline & & & $x ?$ \\
\hline & & & $Y ?$ \\
\hline
\end{tabular}

Note. Letters $A, B, C, X$ and $Y$ refer to the substances which served as the cues being paired with the outcome Miltosis. The digits refer to the outcome magnitude (i.e. number of symptoms of Milthosis . 


\section{Results and Discussion}

Figure 1.1 displays the curusal ratings atest for cues $A, B, C, X$ and $X$. The top panel displays the causal ratings at test for group Bloking, and the lower panel displays the causal rating at testor for control group.
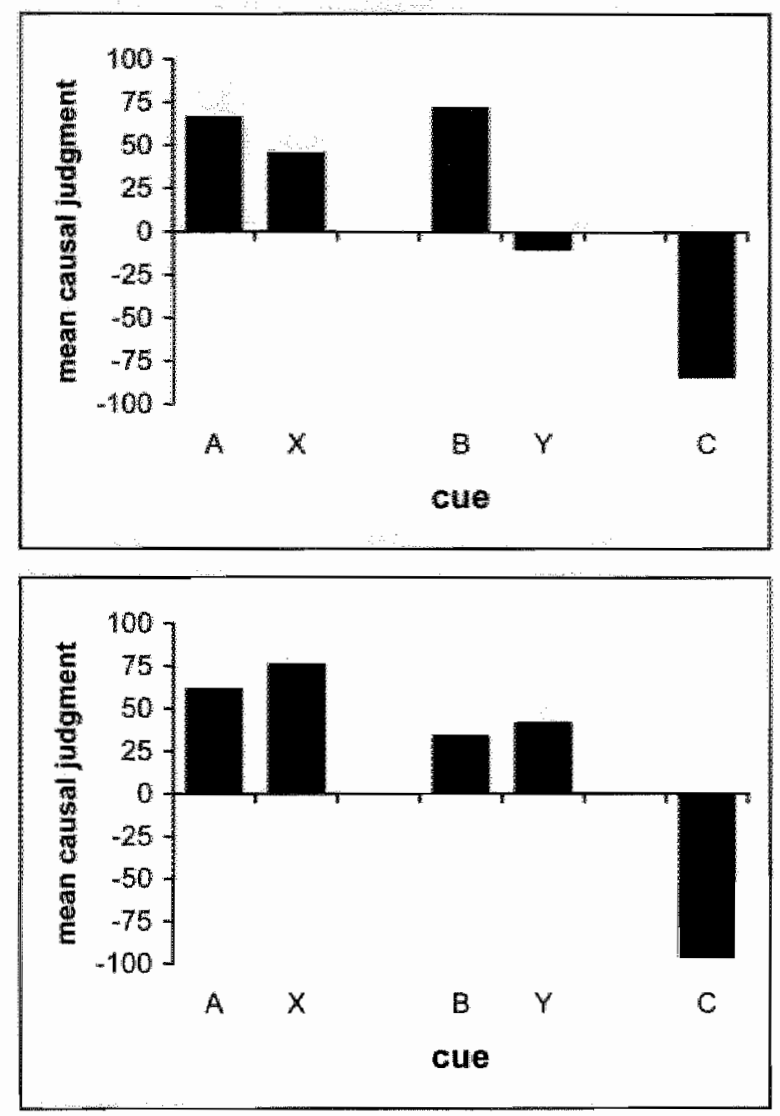

Figure 1.1. Mean causal judgments for cues $A, B, X, Y$, and $C$ as measured at test. The top panel displays causal ratings for the participants in group Bllocking, and the lower panel refers to the causal ratings for the participants in the control group.

To test whether the experimental manipulation had led to blocking of causal judgment of cue $X$ and cue $Y$, contrasts of causal judgment between cues $A$ and $X$, and between cues $B$ and $Y$ were calculated for each group. Since $Y$ is a redundant cue, as opposed to $X$, it was predicted that only the contrast $B-Y$ would differ between groups and 
that this particullar contrast should be larger than the $A-X$ contrast. To test this prediction, a repeated measures analysis of variance (ANOVA) was conducted, with Group (Blocking versus Control) as the between-subjects factor and Contrast ( $\mathrm{AX}$ versus $\mathrm{BY}$ ) as the withinsubject factor. A main effect of Group was found, $F(1,30)=10.59, p<.01$, indicating differential levels of causal ratings between the groups. A main effect of Contrast was found, $F(1,30)=6.34, p<.05$, indicating differential levels of blocking of causal judgments to cues $X$ and $Y$. Moreover, a significant interaction between these factors was found, $F(1,30)$ $=4.25, p<.05$, which indicates that the difference in the degree of blocking to $\mathrm{X}$ and $\mathrm{Y}$ may have been specific for one particular group, and that the difference in contrasts (AX versus BY) between groups may have been limited to one of the two contrasts. To determine the exact nature of this interaction, two paired samples $t$ tests were conducted, comparing contrasts $\mathrm{AX}$ and $\mathrm{BY}$ for each separate group. No significant difference between contrasts $\mathrm{AX}$ and $\mathrm{BY}$ was found for the participants in the Control group, $t(15)=0.53, n s$, whereas a significant difference was found for the participants in the Blocking group, $t(15)$ $=2.53, p<.05$. Additionally, two independent samples $i$ tests were conducted comparing contrasts $\mathrm{AX}$ and $\mathrm{BY}$ separately between groups. A near significant difference was found for contrast $\mathrm{AX}, t(30)=2.00, p=.05$, but a large significant difference was found for contrast $\mathrm{BY}, t(30)=4.73, p<.001$. The results of interest thus indicate that participants in the experimental blocking group demonstrated strong blocking to cue $\mathrm{Y}$, but unblocking to cue $\mathrm{X}$, as predicted by the RW model.

\section{Experiment 2}

In experiment 1 it was found that when the outcome magnitude on AX-O compound trials exceeds the outcome magnitude on the preceding A-O trials, this leads to unblocking of causal judgment. To test the reliability of this effect, an attempt to replicate this pattern of results was made using a simultaneous and backward blocking procedure. A simultaneous blocking procedure concerns the intermixed presentation of $\mathrm{A}-\mathrm{O}$ and $\mathrm{AX}-\mathrm{O}$ trials in a single learning phase. This single-stage blocking design has been found to render effective blocking of human causal learning and can be accommodated by the RW model (see Cramer et al., 2002; Price \& Yates, 1995). Therefore, both blocking and unblocking of causal judgment should also be obtained with this procedure.

Blocking of human causal learning has also been successfully demonstrated using a backward blocking paradigm (Chapman, 1991; Larkin, Aitken, \& Dickinson, 1998; Shanks, 1985). In this procedure, the AX-O compound trials precede the elemental A-O trials. According to the RW model, such a design should not lead to the blocking of causal judgment of cue $X$, as in the initial phase both elements of the $A X$ compound will be asso- 
ciated with the outcome, and the associative strength of a cue cannot change on trials where this particular cue is absent. The successful demonstration of backward blocking in human cansal learning thus camnot be explained in terms of the RW model. However, Van Hamme and Wassenman (1994) have argued that a relativelly simple modification of the RW model can overcome its limitations in predicting backward blocking. They suggested that on trials in which cue $X$ is expected but absent, the salience of $X$ receives a negative value, hence losing associative strength on the $\mathrm{A}-\mathrm{O}$ trials following the $\mathrm{AX}-\mathrm{O}$ compound trials. Such retrospective revaluation of cue $\mathrm{X}$ is described by

$$
\Delta V_{X}=-\alpha_{X} \beta_{O}\left(\lambda-\sum V\right)
$$

where $\alpha_{X}$ thus receives a negative value on trials where cue $X$ is expected but absent. This modified RW model explicitly predicts that not only blocking, but also unblocking should be observed when employing a backward blocking procedure.

\section{Method}

Participants. A total of 48 undergraduate students (43 women; 5 men) from the Maastricht University were recruited through posted advertisements $(M$ age $=19.8$ years, $S D=2.1$ ). They received course credits upon completion of the experiment.

Apparatus. The same apparatus was used as described in experiment 1 .

Procedwre and Design. Participants were randomly assigned to one of three different groups of equal size $(n=16)$ : Simultaneous Blocking group, Backward Blocking group or Control group. Participants in the present experiment performed the exact same task as described for experiment 1 . As opposed to experiment 1 , no trials were included displaying that exposure to a cue $C$ leads to the absence of any symptoms of Miltosis, Substance mames Nafteen, Tramine, Glexus and Phenidaat were counterbalanced assigned to serve as cues $A, B, X$, or $Y$. Participants in the simultaneous blocking group received $8 \mathrm{~A}-\mathrm{O}$ and $8 \mathrm{~B}-\mathrm{O}$ trials, and $8 \mathrm{AX}-\mathrm{O}$ and $8 \mathrm{BY}-\mathrm{O}$ compound trials intermixed in a single learning phase. Trials were presented in a random order for each separate participant with the restriction of no more than three consecutive trials of the same type. Participants in group Backward Blocking received the $16 \mathrm{AX}-\mathrm{O}$ and $\mathrm{BY}-\mathrm{O}$ compound trials before the $16 \mathrm{~A}-\mathrm{O}$ and $\mathrm{B}-\mathrm{O}$ trials. For each of the two phases, trials were presented in a random order for each separate participant. The control group received the exact same manipulation as described in experiment 1 . At test, participants again had to indicate to what degree they believe that a presented single substance (A, B, X, or Y) causes the development of symptons of Miltosis. The experimental design of experiment 2 is summarised in Table 1.2. 
Table 1.2

Design of experiment 2.

\begin{tabular}{|c|c|c|c|}
\hline & & Phase & \\
\hline & One & Two & Test \\
\hline \multirow{4}{*}{ Simultaneous Blocking } & $A-3$ & & A? \\
\hline & $B-3$ & & $\mathrm{~B} ?$ \\
\hline & $A X-5$ & & $x ?$ \\
\hline & $B Y-3$ & & $Y ?$ \\
\hline \multirow{4}{*}{ Backward Blocking } & $A X-5$ & $A-3$ & $\overline{A ?}$ \\
\hline & $\mathrm{BY}-3$ & $B-3$ & B? \\
\hline & & & $x ?$ \\
\hline & & & $Y ?$ \\
\hline \multirow{4}{*}{ Control } & $A X-5$ & & A? \\
\hline & $B Y-3$ & & $\mathrm{~B}$ ? \\
\hline & & & $x ?$ \\
\hline & & & $Y ?$ \\
\hline
\end{tabular}

\section{Results and Discussion}

Mean causality ratings for cues $\mathrm{A}, \mathrm{B}, \mathrm{X}$, and $\mathrm{Y}$ are displayed in Figure 1.2. The top panel refers to the causal ratings of group Simultaneous Blocking, the middle panell displays the ratings of the participants from group Backward Blocking and the lower panel refers to the ratings of the Control group.

As in experiment 1, for each group contrasts of causal judgments at test between cues $A$ and $X$ and $B$ and $Y$ were calculated. Planned comparisons were conducted, comparing the experimental groups (Simultaneous Blocking and Backward Blocking) with the Control group for the degree of blocking of causal judgment of cues $\mathrm{X}$ and $\mathrm{Y}$. Comparing group Simultaneous Blocking with Control, a difference was found in contrasts (AX versus $B Y), F(1,30)=4.33, p<.05$. Further, a significant main effect of Group was found, $F(1$, $30)=12.86, p<.01$, indicating differential levels of causal ratings between the two groups. Moreover, an interaction was found, $F(1,30)=12.61, p<.01$, suggesting that the difference in contrasts is limited to a single group, and that the difference in contrasts between groups is limited to a particular contrast. To further examine this interaction, paired samples $t$ tests were conducted comparing the $\mathrm{AX}$ and BY contrasts within each group (i.e., Simultaneous Blocking versus Control). These analyses revealed that a difference between contrasts $\mathrm{AX}$ and $\mathrm{BY}$ was only significant for group Simultaneous Blocking, $t(15)=3.25$, 
$p<.01$, but not for the participants in the Control group, $t(15)=1.47$, ns. As can be inferred from Figure 1.2, there was much stronger blocking for cue $\mathrm{Y}$ than for cue $\mathrm{X}$ in the Simultaneous Blocking group. Additionally, independent samples $t$ tests were conducted comparing the contrasts $A X$ and $B Y$ separately between groups. No significant difference in contrast $\mathrm{AX}$ was found between groups Simultaneous Blocking and Control, $t(30)=.41$, $n s$, but a large significant difference was found for contrast $\mathrm{BY}, t(30)=4.65, p<.001$.
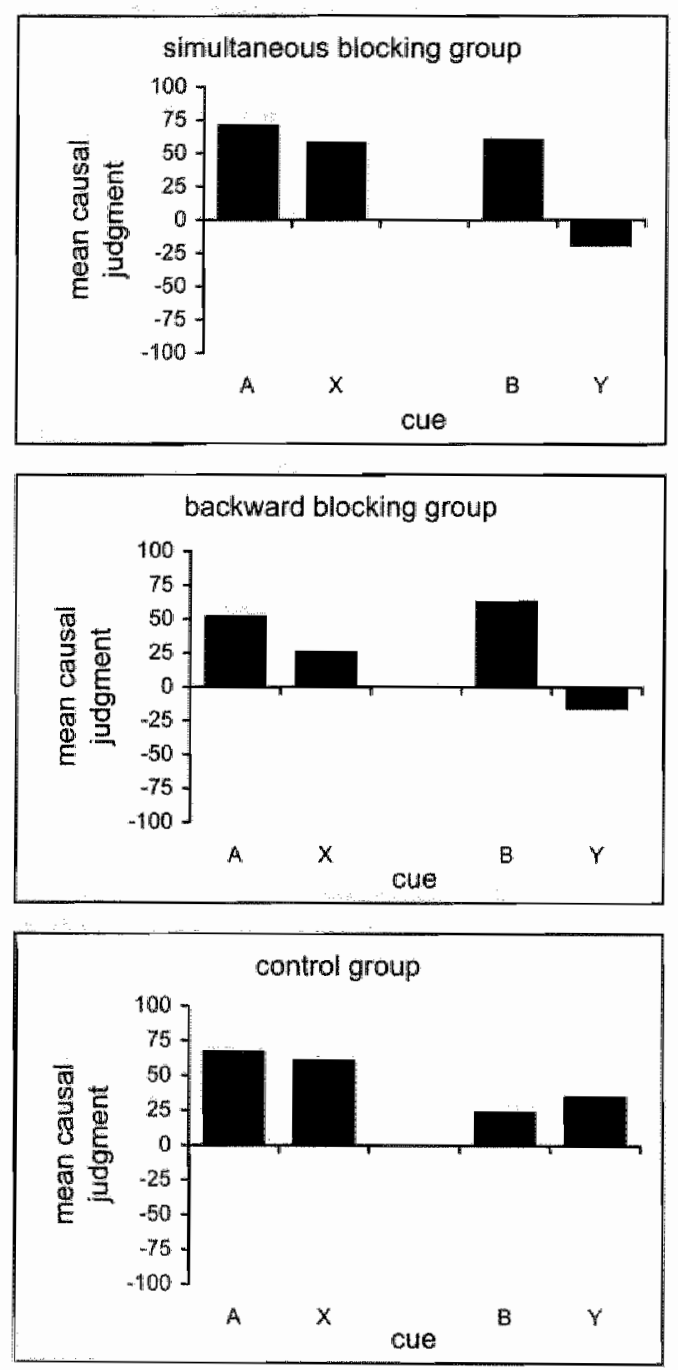

Figure 1.2. Mean causal judgments at test for cues $A_{*} B, X_{t}$ and $\gamma$. The top panel refers to the causal ratings for participants in group Simultaneous Blocking, the middle panel displays the test results for group Backward Blocking and the lower panel refers to the causal ratings at test for the control group. 
Next, group Backward was compared with group Control. No overall effect of contrasts was found, $F(1,30)=1.87, n s$. A main effect of Group was found, $f(1,30)=17.36$, $p<.001$, indicating differential levels of causality ratings between the groups. Although an overall effect of contrasts was absent, a significant interaction between Contrast (AX versus BY) and Group was found, $F(1,30)=7.48, p<.01$, indicating that there is a discrepancy in blocking to $\mathrm{X}$ and $\mathrm{Y}$, but potentially limited to one of the two groups, and that the difference in contrasts between the groups Backward Blocking and Control may be limited to one of the two contrasts. A paired samples $t$ test was conducted comparing the $A X$ and BY contrasts within group Backward Blocking. A significant difference was found, $t(15)=$ $2.32, p<.05$. As can be seen in Figure 1.2, similar to group Simultaneous Blocking, participants in group Backward Blocking displayed much stronger blocking of causal judgment for cue $\mathrm{Y}$ than for cue $\mathrm{X}$. Additionally, two independent samples t tests were conducted, comparing groups Backward Blocking and Control for each contrast (AX versus BY) separately. No difference for contrast AX was found between these two groups, $(30)$ $=1.01, n s$, but a significant difference was found for contrast $\mathrm{BY}, t(30)=5.41, p<.01$.

Finally, group Backward was compared with group Simultaneous. Only an effect of Contrast (AX versus BY) was found, $F(1,30)=15.22, p<.01$, other $F s<1$. The results of interest thus indicate that, as in experiment 1 , outcome magnitude affects blocking of causal judgment. Although these results are specifically predicted by the (modified) RW model, there is an alternative explanation for the present demonstration of both blocking and unblocking. It is conceivable that the participants deduced causality of cues $\mathrm{X}$ and $\mathrm{Y}$ retrospectively. At the time of testing, for example, they could have deduced the causal status of cue $\mathrm{X}$ by performing simple arithmetic after the observation of the contingencies in the form of:

if $A=3$ symptoms of Miltosis, and $A+X=5$ symptoms of Miltosis,

then $\mathrm{X}$ must be regarded as a potential cause of Miltosis leading to at least 2 symptoms.

\section{Experiment 3}

In experiments 1 and 2 , it was shown that unblocking can be observed when varying the outcome magnitude. Although this pattern of results can be explained by the (modified) RW model of associative learning, an alternative explanation is that both blocking and unblacking of causal judgment are the result of deductive reasoning. Some evidence has al- 
ready been found for the latter view. De Houwer et al. (2002) demonstrated that blocking was more pronounced when a suboptimal outcome was presented on both the A-O and AX $-O$ trials. Smilar results were found by Lowibond et al. (2003). According to De Houwer et al., with a binary outcome (ie, outcome present or absent), one cannot logically infer that a cue $\mathrm{X}$ is truly redundant and hence blocking should be incomplete (see also Cheng, 1997). This celling-effect is not present in the case of suboptimal outcomes and thus allows for a much stronger blocking effect. Clearly the RW model camnot account for these results, but the assumption that causal judgment relies on retrospective deductive reasoning can.

Another type of finding that is difficult to explain in terms of associative learning but can be accounted for by the notion that causal judgment reflects deductive reasoning, is that blocking of causal judgment does not necessarily rely on trial-by-trial experience, but also occurs when presenting the cue-outcome contingencies in a summarised format (see e.g., Van Hamme \& Wasserman, 1993). If causal learning indeed reflects deductive reasoning, then it should not matter in what order, or even how knowledge about cue-outcome contingencies is acquired. Therefore, it was tested whether the same pattern of results, that is, blocking and unblocking of causal judgment, could be obtained when describing the specific contingencies in a summarised format.

\section{Method}

Participants. A total of 16 participants ( 5 women; 11 men) were recruited by posted advertisements at the Maastricht University ( $M$ age $=26.7$ years, $S D=9.5$ ). None of these participants studied (or had studied) psychology at the time of testing. All participants took part on a voluntary basis.

Procedure and Design. The experiment was approved by a local ethical committee. A within-subjects design was used. All participants were tested individually and received generally the same instructions as in the previous experiments, but in the present experiment were printed on paper. Again, participants had to play the role of a medical researcher investigating the cause of Millosis. In contrast to experiments 1 and 2 the results of the medical research were now described in a summary (translated from Dutch):

Exposure to a substance $A$ always led to the development of 3 symptoms of Miltosis.

Exposure to substances $A$ plus $X$ always led to the development of 5 symptoms of Miltosis.

Exposure to a substance B always led to the development of 3 symptoms of Miltosis.

Exposure to substances $B$ plus $Y$ always led to the development of 3 symptoms of Militosis.

Exposure to substances $\mathrm{C}$ and $Z$ always led to the development of 3 symptoms of Miltosis.

Exposure to a substance D always led to the development of 0 symptoms of Miltosis. 
Participants received the instruction to memorise this summary, and were not allowed to take any notes. After indicating they had memorised the research results, the participants received a booklet of 7 pages. On each page the name of a single substance was presented $(A, B, C, D, X, Y$, or Z) and for each substance they had to indicate on a $200 \mathrm{~mm}$ VAS, ranging from -100 at the extreme left (absolutely certain of NO symptoms of Miltosis) to 0 in the middle (no idea) to 100 at the extreme right (absolutely certain of symptoms of Miltosis), the degree to which they believe that exposure to the presented substance causes the development of symptoms of Miltosis.

\section{Results and Discussion}

Figure 1.3 displays the causal judgments to the cues $A, B, C, D, X, Y$, and $Z$ at test. $A$ s in experiments 1 and 2, contrasts of causal judgments at test between cues $A$ and $X, B$ and $Y$, and $\mathrm{C}$ and $\mathrm{Z}$ were calculated. Conducting a repeated measures $\mathrm{ANOVA}$, it was found that the contrasts $(\mathrm{AX}, \mathrm{BY}$, or $\mathrm{CZ})$ differed, $F(2,30)=10.80, p<.001$. Subsequent paired samples $t$ tests revealed a near significant difference between contrasts $\mathrm{AX}$ and $\mathrm{CZ}, t(15)=$ 2.14, $p=.05$, a significant difference between contrasts $\mathrm{BY}$ and $\mathrm{CZ}, r(15)=3.97, p<.01$, and a significant difference between contrasts $\mathrm{AX}$ and $\mathrm{BY}, t(1,5)=2.80, p<.05$. These results indicate that some blocking to $X$ did occur, but in agreement with experiments 1 and 2 , blocking to cue $Y$ exceeded blocking of causal judgment of cue $X$. So again, both blocking and unblocking was demonstrated. It is clear that the present results cannot be explained in terms of associative learning as the contingencies were not presented trial-bytrial but were communicated through the presentation of a summary of the cue-outcome contingencies.

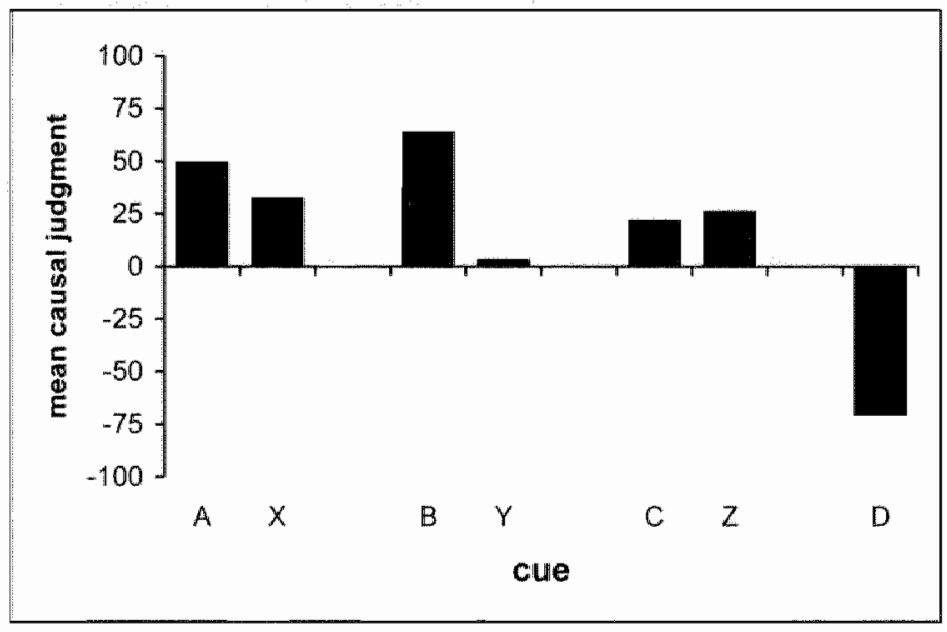

Figure 1.3. Mean causal judgments for cues $A, B, C, D, X, Y$, and $Z$. 


\section{General Discussion}

In a series of three experiments it was found that outcome magnitude affects cue competition effects in human causality detection, that is, when the outcome magnitude on AX-O trials exceeds the magnitude of the $\mathrm{A}-\mathrm{O}$ trials, less or even no blocking of causal judgment will occur. This pattern of results can be explained by associative models of causal learning, such as the (modified) RW model (see Van Hamme \& Wasserman, 1994). However, these models all predict that causality detection depends on the trial-by-trial experience of cue-outcome contingencies and thus fail to accommodate the successful demonstration of blocking and unblocking of causal judgment when the cue-outcome contingencies are described rather than experienced as was the case in experiment 3. Clearly, human causal learning does not necessarily reflect associative learning processes.

It can be argued that the present results are best explained in terms of deductive reasoning, that, is, after the observation of the specific cue-outcome contingencies one performs simple arithmetic to deduce the causal status of the target cue. In all three experiments, cue A led to the development of 3 symptoms of Miltosis, and the compound AX led to the development of 5 symptoms of Miltosis. When at test one thas to indicate the causal status of $X$, participants could have simply computed the number of symptoms that $X$ will cause by subtracting the number of symptoms caused by the compound $\mathrm{AX}$ with the number of symptoms caused by A alone. Of course, on the basis of the present study one cannot discand the possibility that associative learning may still play a considerable role in human causality detection. Shanks (1991), for example, has argued that causal judgments based on newly experienced cue-outcome contingencies directly reflects associative learning, whereas causal judgments based on described contingencies involves judgments based on causal beliefs, which in turn may have been established through associative learning. Quite similarly, Price and Yates (1995) have argued that online causal learning tasks involves associative learning processes, whereas causal judgments in case of described contingency information involves higher-order rule based processes.

However, even when cue-outcome contingencies are experienced online, deductive reasoning processes have still been found to play an important role in demonstrating blocking of causal judgment. Recently, De Howwer and Beckers (2003) demonstrated that blocking of causal judgment is impaired when participants had to perform a secondary cognitive task. It was hypothesised that this secondary task should specifically interfere with any effortful reasoning processes, and thus when blocking of causal judgment does not depend on such reasoning this task should not have affected blocking. Clearly, it did. Moreover, secondary task difficulty modulated the degree of blocking, that is, the more difficult the 
secondary task the less blocking was observed. De Houwer and Beckers thus argue that human causal learning depends at least partially on deductive reasoning processes. This notion is in agreement with the causal-model theory proposed by Waldmann (2000; see also Waldmann \& Holyoak, 1992). Waldmann demonstrated that causality detection depends not only on the observation of cue-outcome contingencies, but also on a prioni knowledge how to interpret these contingencies, and thus argues that causal learning does not reflect the operation of associative learning processes. Waldmann and Holyoak even suggested that certain associative learning phenomena might be reduced to higher order causal induction. Indeed recently, Lovibond (2003) demonstrated that retrospective revaluation of both Pavlowian conditioned skin conductance responding and causal judgments does not necessarily depend on the trial-by-trial experience of cue-outcome contingencies. The experimental task comprised an aversive conditioning procedure where the CSs were coloured squares presented on a computer screen and the outcome was the administration of an electric shock. Repeatedly pairing a compound $\mathrm{AB}$ with shock led to conditioned skin conductance responding and strong expectancies of the shock as rated by the participants with each presentation of the compound. Subsequent nonreinforced exposure to A alone led to stronger conditioned skin conductance responding to cue $\mathrm{B}$ and increased expectancy ratings for cue B at test. Such a release from overshadowing however, was also observed when after the compound $\mathrm{AB}-\mathrm{O}$ trials participants were simply told that $A$ is safe (i.e., cue $A$ is not followed by the administration of an electric shock). Therefore, Lovibond proposes that retrospective revaluation of both human Pavlovian conditioned responding and causal judgment depends on reasoning processes on the basis of propositional knowledge, whether such knowledge is acquired through experience or acquired through communication. Considering these results and the results of the present study, the present challenge lies in determining more precisely to what degree human causality detection depends on effortful reasoning processes. 
EXCESSIVE APPETTE 


\section{PART I}

\section{THE PAVLOVIAN NATURE OF AN EXCESSIVE APPETITE FOR DRUGS AND ALCOHOL}

Actually, it only takes one drink ro get me loaded. Trowble is, I can "t remember if it's the thirteenth or fourteenth.

George Burns 
EXCESSVE APpETTE 


\title{
CHAPTER 2
}

\section{THE EFFICACY OF CUE EXPOSURE WITH RESPONSE PREVENTION IN EXTINGUISHING DRUG AND ALCOHOL CUE REACTIVITY**}

\begin{abstract}
It has been suggested that drug cue-elicited urges and psychophysiological reactions are the result of Pavlovian conditioning processes and that it should be possible to extinguish these responses with cue exposure with response prevention. It has already been shown that subjective cuemelicited urges can be extinguished, but it is unclear whether this is also true for psychophysiological cue reactivity. This was tested in the present study in a heterogeneous sample of drug and alcohol dependent patients. It was found that cue-elicited urges can indeed be extinguished. However, such a clear pattern of extinguished cue reactivity was not found for the psychophysiological measures. Furthermore, the extinction of drug urges was not specific for cue exposure treatment. It is concluded that cue-elicited psychophysiological arousal does not underlie subjective cue reactivity and may not reflect Pavlovian conditioned drug responding.
\end{abstract}

\footnotetext{
"This chapter is an adapted version of: Havermans, R.C., Mulkens, S., Nederkoom, C., \& Jansen, A. (2004). The efficacy of cue exposure with response prevention in extingzishing drug and alcohol cue reactivify. Manuscript submitted for publication.
} 
Associative leaming has frequently been suggested to underlie the development and maintenance of addictive behaviour. Pavlovian learning models of addiction have received considerable research interest in the past 25 years. One of the most prominent of these theories is the compensatory response model by Siegel (1983). According to Siegel, drug consumption disrupts homeostatic functioning. As one learns that certain drug-related cues predict drug intake, one learns to anticipate the drug effect. Drug cues are thus thought to function as conditioned stimuli being associated with drug use. Conditioned drug responding as a result of this association is termed cue reactivity and according to Siegel should comprise psychophysiological responding opposite to the effect of the drug, thus limiting the disruptive effect of drug use on homeostatic functioning. As such reactivity in most cases will resemble an aversive withdrawal state this reactivity will be experienced subjectively as urges to use drugs in order to alleviate this state. Although several studies support the notion that drug cue reactivity is opposite to the effect of the drug in question (see e.g., Siegel \& Ramos, 2002), this is not always necessarily so. Therefore, Stewart, de Wit and Eikelboom (1984) proposed that the primary cue-elicited drug response is isodirectional to the effect of the drug. Drug cues are thus thought to remind one of the rewarding effects of the drug and hence increase incentive motivation for drug use. In 1999, Carter and Tiffany presented a meta-analysis systematically reviewing most studies concerning the measurement of psychophysiological cue reactivity and came to the conclusion that generally there is more support for the conditioned incentive model. However, they also proposed an alternative explanation for the pattern of psychophysiological reactivity found in substance dependent patients. They noted that across different types of drugs psychophysiological reactivity is very similar. This finding does not confer with the associative models of addiction that explicitly predict that psychophysiological cue reactivity should be drug specific, that is, being either isodirectional or opposite to the effects of the specific drug in question. Carter and Tiffany thus state that exposure to cues leads to a general arousal response, particularly characterised by an increase in skin conductance level and heart rate.

Exposure to drug cues is undoubtedly stressful for substance dependent patients. It immediately leads to an internal conflict characterised by the desire to use drugs and the simultaneous motivation to remain abstinent. When one is exposed to such a stressor, this leads to the release of catecholamines (epinephrine and norepinephrine) that increase blood pressure, heart rate, skin conductance level, and induce peripheral vasoconstriction (Grunberg \& Singer, 1990). Carter and Tiffany (1999) suggest that this arousal response to drug cues can be explained in terms of the nonassociative model of drug use and drug urges proposed by Tiffany (1990). According to Tiffany, drug cues activate a cognitive drug action schema allowing for the automatic use of drugs. Impeding this action schema leads to frustration and accompanying activation of higher order cognitive processes to ensure drug consumption. Exposure to cues thus leads to mental stress, which is reflected by a subjectively experienced turge and psychophysiological responding indicative of arousal. How- 
ever, although a general arousal response is not predicted by any of the existing associative models of drug addiction, this does not necessarily imply that such a response pattern cannot be the result of Pavlovian conditioning processes. Wise and Bozarth (1987) argue that the primary effect of all drugs of abuse is their psychomotor stimulating, or arousing effect. It is conceivable that drug cues are associated with this arousing effect and hence come to ellicit conditioned arousal responding that contributes to the experience of drug urges. Indeed, autonomic arousal can be subject to conditioning (Johnson \& Anderson, 1990). For example, Dawson and Biferno (1973) demonstrated that participants could learn to discriminate between a neutral tone $A$ paired with an electric shock and another neutral tone $B$ that was not paired with the administration of the shock, such that presentation of tone $A$ alone came to elicit an increase in skin conductance responding.

It has been argued that psychophysiological cue reactivity is indeed a conditioned drug response and that it should be possible to extinguish such responding by applying cue exposure with response prevention (CERP). CERP refers to the treatment in which the patient is repeatedly exposed to cues related to his/her addictive behaviour without being able to submit to this behaviour. This procedure elicits strong urges, but with repeated exposure the cues lose their predictive value and hence craving gradually extinguishes (Rankin, Hodgson, \& Stockwell, 1984). Although it is clear that CERP can lead the successful extinction of subjective cue reactivity (i.e., craving), it has remained unclear to what degree CERP can affect psychophysiological cue reactivity as very few CERP studies have incorporated such psychophysiological measures (see also Carter \& Tiffany, 1999). In the present study, it was hypothesised that both cue-elicited psychophysiological arousal responding and craving can be extinguished with CERP. This hypothesis was tested in a heterogeneous sample of different drug or alcohol dependent patients.

\section{Method}

\section{Patients}

The study was approved by a medical ethics committee. All patients in the present investigation signed informed consent prior to their participation in the study.

A total of 70 drug or alcohol dependent patients ( 53 men and 17 women, $M$ age $\approx 37$ years) agreed to take part in the present study, comprising 36 alcohol dependent patients, 15 cocaine dependent patients, 11 opiate dependent patients, 2 psychostimulant dependent patients, 4 cannabis dependent patients, and 2 benzodiazepine dependent patients. All patients were diagnosed as being alcohol or drug dependent according to DSM-IV criteria and were recruited from a substance abuse treatment facility in Heerlen, the Netherlands. Patients at this treatment centre have a length of stay varying from 14 to 270 days. The standard treatment program at this facillity includes group therapy sessions, individual counselling, coping skills training, social skills training, creativity therapy 
and aftercare plaming. Patients were eligible for participation in the present study if being at least 18 years old and detoxiffed. Patients were excluded from the study if they were on urge-inhibiting medication (e.g, naltrexone, accamprosate, or benzodiazepines), or displayed comorbidity of psychotic symptoms, suicidal ideation, or PTSD.

\section{Treaments}

Patients were randomly assigned to one of two treatments: Relaxation training (RT), or CERP. The two forms of treatment were encoded and with a random number table a random sequence of treatment assignment was established. With this randomisation procedure, potentially interfering patient and timing variables are neutralised since the chance of being appointed to one of the two forms of treatment is equal for each patient and the chance of being appointed to a certain position within the random sequence is equal for each of the two forms of treatment (Cook \& Campbell, 1979; Barker, Pistrang, \& Elliott, 1994). This randomised design rules out potential selection bias, but there may be leakage between conditions. In other words, patients may share their treatment experiences. Further, attrition due to drop-out may affect equivalence of the two groups.

Each treatment consisted of eleven 1-hour sessions in which the patients received either RT or CERP. Rohsenow, Monti and Abrams (1995) have argued that approximately 6 hours of cue exposure should suffice in extinguishing cue reactivity. All sessions took place during weekdays spaced across a period of 4 to 5 consecutive weeks. Both treatments were added to the standard treatment program of the substance abuse treatment facility.

Treatment providers with at least a Master's degree in Mental Health or with equivalent work experience administered the two forms of treatment. Training manuals, weekly supervision and random listening of tape recorded treatment sessions by one of the investigators ensured treatment integrity. Each treatment provider made a report of every session they conducted.

Cue exposure with response prevention (CERP). Patients were told that the CERP was intended to decrease the urge to drink alcohol or use drugs in situations related to their addictive behaviour, hence reducing the probability of a relapse after treatment. The first two sessions consisted of explaining the rationale of the treatment and identifying drug-related cues and triggers. Each of the 9 following expostre sessions consisted of: (1) baseline urge rating, (2) active exposure to the drug-related cues and (3) imaginal exposure.

During the exposure sessions, the urge to drink alcohol or use a specific drug was assessed every ten minutes on 100-mm Visual Analogue Scales (VASs) ranging from 0 "no urge at all" to 100 "near irresistable urge". A schematised representation of the rationale lay visible on the table, in order for the therapist to refer to the rationale when deemed necessary. Following each session, the therapist briefly evaluated the session with the patient.

No homework was prowided as to limit the danger of a lapse (and subsequent exclusion from the study) in between sessions and as to limit the probability of leakage. To further limit the probability of leakage, all patients were explicitly instructed to discuss treatment experiences individually with treatment staff member and not with any of their fellow patients.

Relaxation training (RT). Patients randomly assigned to group $\mathrm{RT}$ received a similar amount of individual treatment sessions as the patients receiving CERP, but comprised training in different relaxation techniques. $\mathrm{RT}$ is incorporated in many standard treatment programs and its. rationale has high credibility, but it has been found to have no long term effect on alcohol and drug 
use outcomes and is not gimed at extinguishing or directly affecting craving (see Klajner, Hartman, \& Sobell, 1984; Drummond \& Glautier, 1994). As this form of treatment is sumiciently different from CERP, it was incorporated in the present study to serve as a placebo control treatment.

Patients were told that RT is intended to reduce the chance of rellapse by managing one's experience of negative emotions due to stressful life events. In the first two sessions, patients were taught to identify stressfull events and to become aware of their physiological reactions to such events. In the following nine 1-hour sessions, they were taught several different relaxation techniques. As for CERP, no homework was provided.

\section{Cue reactivity assessment}

Directly before and after treatment (CERP or RT), all patients received a cue reactivity assessment. This comprised the recording of skin conductance level (SCL), skin temperature (STMP), changes in finger pulse amplitude (FPA), thus measuring either peripheral vasoconstriction or vasodilatation (see Nederkoorn, Smulders, Havermans, \& Jansen, 2004), and interbeat interval (IBI) as a measure for heart rate using Psylab 7 measuring devices (Contact Precision Instruments, UK). SCL was measured with two $\mathrm{Ag}-\mathrm{AgCl}$ electrodes, placed on the thenar and hypothenar eminences of the palm of the nondominant hand. STMP was measured with a thermistor placed on the back of the nondominant hand. FPA and IBI were determined with the aid of finger pulse photoplethysmography, with the sensor placed on the indexfinger of the nondominant hand. Next to the measurement of psychophysiological cue reactivity, momentary subjective cue-elicited urge was measured with the 100-mm VASs.

The patient was briefly informed about the general procedure of the measurement and was then asked to wash his hands with water. Next, he was connected to the psychophysiological measuring devices and was asked to sit as still as possible during the entire assessment. The assessment started with a 2-min baseline recording of psychophysiological reactivity. This baseline measurement was intended to bring the diverse psychophysiological measures to resting level. After these 2 minutes, the patient was asked to indicate on a VAS the momentary urge to use a given drug, after which he was given a small bar of soap. He was instructed to focus his attention on this bar of soap for a period of 5 minutes (i.e., holding it, and looking and smelling at it), after which he had to indicate his drug or alcohol urge on a VAS again. After exposure to the neutral soap cue, the patient was exposed to personalised drug or alcohol cues for another 5 minutes. During this cue exposure, the patient was instructed to concentrate on the cues, to look at the cues, handle the cues and to smell at the cues (at least if there was anything to smell). After this cue exposure, the patient rated his drug or alcohol urge for a final time on a VAS.

\section{Results}

Of the 70 patients that were initially recruited, 6 patients dropped out before actual participation in the present study. The main reason for this attrition being relapse and receiving subsequent mandatory discharge from the treatment facility. The results are displayed in 
Table 2.1, showing mean reactivity withen exposed to the neutral cue and the drug-related cues at the cue reactivity assessments before and after treatment for each group.

Table 2.1

Mean (+ SD) skin conductance level (SCL), skin temperature (STMP), interbeat interval (IBI), finger pulse amplitude (FPA) and urge score when exposed to the neutral cue (N) and the drug cue (C) at pre-treatment and post-treatment cue reactivity assessment for each group separately (group CERP versus RTT.

\section{GROUP}

\begin{tabular}{|c|c|c|c|c|c|c|c|c|}
\hline & \multicolumn{4}{|c|}{ CERP } & \multicolumn{4}{|c|}{$\mathrm{RT}$} \\
\hline & \multicolumn{2}{|c|}{$\begin{array}{c}\text { Pre-treatment } \\
(n=33)\end{array}$} & \multicolumn{2}{|c|}{$\begin{array}{l}\text { Post-treatment } \\
(n=18)\end{array}$} & \multicolumn{2}{|c|}{$\begin{array}{l}\text { Pre-treatment } \\
\qquad(n=31)\end{array}$} & \multicolumn{2}{|c|}{$\begin{array}{l}\text { Post-treatment } \\
\quad(n=20)\end{array}$} \\
\hline & $\mathbf{N}$ & $\mathrm{C}$ & $\mathbb{N}$ & C & $\mathbf{N}$ & C & $\mathbf{N}$ & C \\
\hline \multirow[t]{2}{*}{ SCL } & 5.18 & 6.55 & 3.76 & 471 & 7.19 & 8.71 & 6.41 & 7.75 \\
\hline & $(2.70)$ & $(3.28)$ & $(2.01)$ & $(2.25)$ & $(4.25)$ & $(4.44)$ & $(4.75)$ & $(4.54)$ \\
\hline \multirow[t]{2}{*}{ STMP } & 29.70 & 29.69 & 30.35 & 30.61 & 30.30 & 30.36 & 30.67 & 30.45 \\
\hline & $(2.74)$ & $(2.87)$ & $(1.91)$ & $(1.77)$ & $(2.30)$ & $(2.30)$ & (2.68) & $(2.88)$ \\
\hline \multirow[t]{2}{*}{$|B|$} & 849.82 & 834.01 & 869.01 & 861.01 & 790.16 & 772.59 & 809.86 & 796.15 \\
\hline & $(111.49)$ & $(111.57)$ & $(108.16)$ & $(107.20)$ & $(135.69)$ & $(136.20)$ & $(100.24)$ & $(92.79)$ \\
\hline \multirow[t]{2}{*}{ FPA } & 27,44 & 24.84 & 30.26 & 27.50 & 28.93 & 25.17 & 32.46 & 29.27 \\
\hline & $(15.74)$ & (14.92) & $(14,01)$ & $(12.45)$ & $(15.88)$ & $(15.17)$ & $(17.78)$ & $(14.78)$ \\
\hline \multirow{2}{*}{ urge } & 8.91 & 42.76 & 7.67 & 29.44 & 8.94 & 40.16 & 5.90 & 20.75 \\
\hline & (13.82) & (30.55) & $(14.05)$ & $(28.14)$ & $(16.54)$ & $(32,88)$ & $(6.86)$ & $(20.16)$ \\
\hline
\end{tabular}

Cue reactivity assessment. To determine whether the cue exposure procedure as conducted during the cue reactivity assessments indeed induced cue reactivity, one sample t-tests were conducted with the contrasts between responding to the drug cues and the neutral cue for each cue reactivity measure as the dependent variables. Due to hardware failures, we failed to record data of 3 patients concerning SCL, 2 patients concerning STMP, 2 patients concerning IBI, and 7 patients concerming FPA at the pre-treatment measurement of cue reactivity.

As hypothesised, the patients demonstrated psychophysiological cue reactivity indicative of an arousal response. Table 2.2 shows the mean cue-elicited changes in SCL, STMP, IBI, and FPA as a function of primary substance dependence. 
Table 2.2

Mean cue-elicited psychophysiological reactivity ( $+\mathrm{SD}$ ) at the pretreatment assessment as a function of primary substance dependence.

\begin{tabular}{lrrrrr}
\hline & \multicolumn{5}{c}{ Primary substance dependence } \\
\cline { 2 - 6 } & \multicolumn{1}{c}{$\begin{array}{l}\text { Alcohol } \\
(n=33)\end{array}$} & \multicolumn{1}{c}{$\begin{array}{c}\text { Cocaine } \\
(n=15)\end{array}$} & $\begin{array}{l}\text { Opiates } \\
(n=10)\end{array}$ & $\begin{array}{r}\text { Psychostimulants } \\
(n=2)\end{array}$ & \multicolumn{1}{c}{$\begin{array}{c}\text { Cannabis } \\
(n=4)\end{array}$} \\
\hline SCL & $1.18(1.13)$ & $1.89(0.94)$ & $1.38(0.92)$ & $1.26(1.04)$ & $2.08(0.48)$ \\
STMP & $-.05(0.63)$ & $-.06(0.60)$ & $0.16(0.64)$ & $-.05(0.49)$ & $0.00(0.28)$ \\
IBI & -20.95 & $-6.75(36.77)$ & -13.08 & $-29.00(14.44)$ & -21.29 \\
& $(35.55)$ & & $(22.56)$ & & $(35.97)$ \\
FPA & $-2.76(5.86)$ & $-3.46(8.78)$ & $-3.62(4.15)$ & $-14.40(9.74)$ & $1.79(10.16)$ \\
\hline
\end{tabular}

It was found that when exposed to drug cues, the patients demonstrated a significant increase in SCL, $t(60)=9.18, p<.001$, no change in STMP, $t(61)=0.22, \mathrm{~ms}$, a significant decrease in IBI that reflects heart rate acceleration, $t(61)=4.40, p<.001$, and a marginally significant decrease in FPA reflecting vasoconstriction, $t(56)=1.73, p=.09$. These results indicate that the cue exposure procedure was successful at elliciting psychophysiological cue reactivity that indeed appears to reflect a general arousal response as has been argued by Carter and Tiffany (1999). Further, it was found that exposure to the drug cues led to a significant increase in subjectively experienced urge to either drink alcohol or use a particular drug, $t(63)=9.70, p^{4}<.001$. However, unexpectedly this cue-elicited urge responding did not correlate with any of the psychophysiological measures of cue reactivity described above, all $p s>.50$. Pearson bivariate correlation coefficients $(*)$ are displayed in Table 2.3 .

Table 2.3

Pearson bivariate correlation coefficients (r) between cue-ellcited urge and SCL. STMP, IBI and FPA at the pretreatment cue reactivity assessment.

\begin{tabular}{l|r}
\hline & \multicolumn{1}{|c}{ Urge } \\
\hline SCL & .09 \\
STMP & -.05 \\
IBI & -.04 \\
FPA & -.14 \\
\hline
\end{tabular}

Extinction of cue reactivity. To determine whether exposure led to the successful extinction of cue reactivity, 2 (Treatment: pre-treatment versus post-treatment) $\times 2$ (Group: CERP versus RT) repeated measures analyses of variance were conducted for each separate cue reactivity measure, except STMP as patients did not demonstrate any cue reactivity for this measure at the pre-treatment cue reactivity assessment. 
A total of $40 \%$ of the patients (26 patients) dropped out before the end of the study lleaving 18 patients in group CERP and 20 in group RT, the main reason for this attrition being, again, a lapse followed by mandatory discharge from the treatment facility. Only the data from patients who completed treatment were entered in the following analyses. Due to hardware failures we also failed to record data from 8 patients concerning SCL, 2 patients concerning IBI, and 9 patients concerning FPA at the post-treatment cue reactivity assessment. The change in cue-elicited changes in SCL, IBI, FPA, and urge ratings across cue reactivity assessments (pre-treatment versus post-treatment) per group (RT versus CERP) is depicted in Figure 2.1.
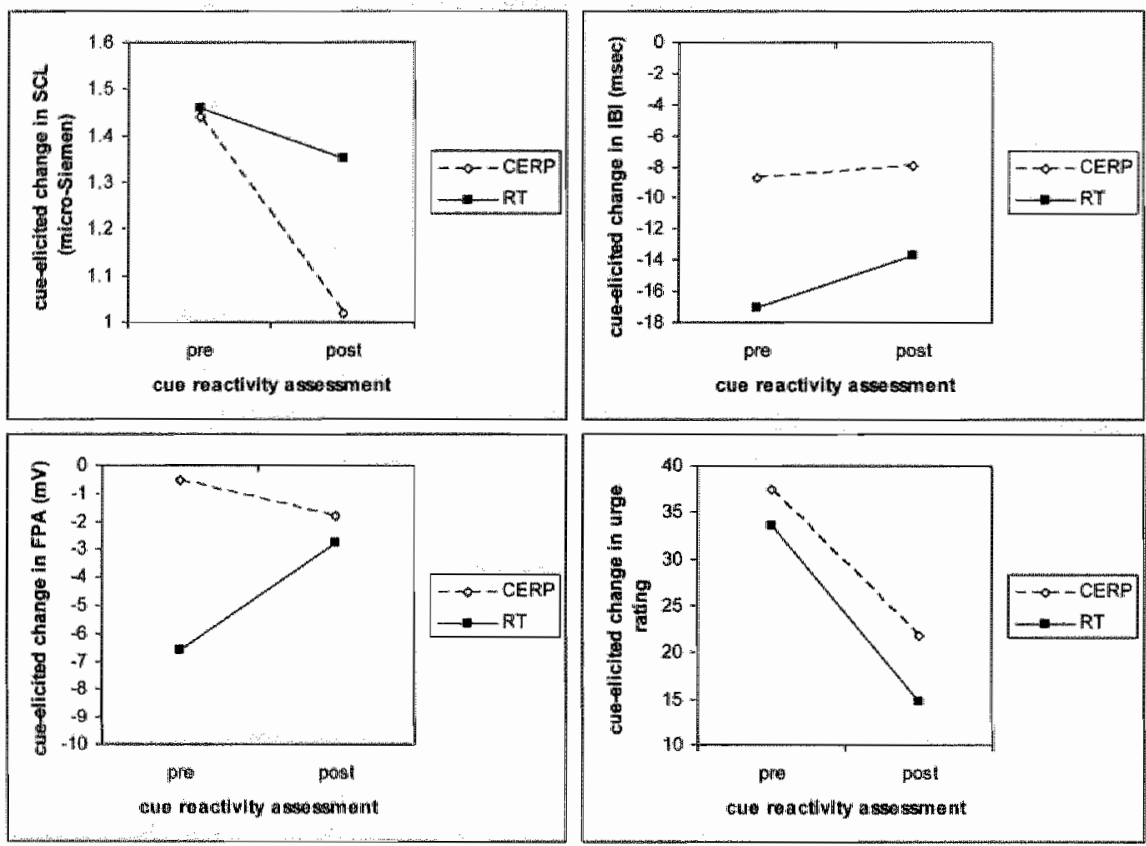

Figure 2.1. Mean cue-elicited changes of $S C L, \| B I, F P A$ and urge ratings at pre- and posttreatment for each separate group (CERP versus RT).

No overall extinction of cue-elicited increase in SCL was found, reflected by the absence of an effect of Treatment, $F(1,28)<1$, and the absence of an interaction between Treatment and Group assignment, $F(1,28)<1$. Finally, no main effect of Group was found for cue-elicited $S C L, F(1,28)=2.07$, ns. A similar pattern of results was found for cue-elicited decrease in IBI. Again, no extinction of this measure was found, that is no main effect of Treatment nor a Treatment $x$ Group interaction was found, $F s(1,34)<1$. Also, no main effect of Group was found, $F(1,34)=1.06$, ns. When examining the cueelicited decrease in FPA, no effect of Treatment was found, $F(1,27)=1.14$, ns. However, 
a Treatment $x$ Group interaction was found, $F(1,27)=4.97, p<.05$. Contrary to our expectations though, this interaction reflects the extinction of cue-elicited vasoconstriction in specifically group RT. This can be attributed to the difference in cue-elicited vasoconstriction between the two groups at the pre-treatment cue reactivity assessment, as can be inferred from Figure 2.1.

No effect of Group was found for FPA, F(1, 27) $=1.91$, ns. In contrast to the psychophysiological measures, cue-elicited urge did extinguish, reflected by a main effect of Treatment, $F(\mathbb{1}, 36)=10.07, p<.01$. However, contrary to our expectations, no Treatment $x$ Group interaction was found, $F(1,36)<1$. Further, no effect of Group was found for cue-elicited urges, $F(1,36)<1$.

\section{Discussion}

In the present study, it was investigated whether cue-elicited psychophysiological arousal and drug or alcohol urges can be extinguished. Associative models of addictive behaviour predict that cue reactivity (both subjective and psychophysiological reactivity) can be extinguished in a drug dependent patient when this patient is repeatedly exposed to cues associated with his/her alcohol or substance use. No clear support for this view was found though. Firstly, psychophysiological cue reactivity appears to reflect a general arousal response. This is in contrast with existing associative models of addictive bellaviour that predict that cue reactivity should be drug-specific. The present study, however, demonstrates that drug cue-ellicited psychophysiological reactions generally comprise an increase in SCL and heart rate, and peripheral vasosconstriction, indicative of an arousal response. Secondly, none of the cue-elicited arousal responses correlated with the degree of cueelicited urges. As associative models of addictive behaviour explicitly state that craving is the subjective interpretation of cue-elicited psychophysiological responding, one would have expected a positive correlation between cue-elicited urges and psychophysiological cue reactivity. Thirdly, psychophysiological cue reactivity did not extinguish at all. The latter result is not a novel finding. For example, O'Brien, Childress, McClellan, and Ehrman (1990) also failed to extinguish psychophysiological cue reactivity in cocaine dependent patients, even after fifteen cue exposure sessions. However, it should be noted that the inability to extinguish cue-elicited psychophysiological arousal does not necessarily imply that such reactivity cannot be regarded as conditioned drug responding as some forms of conditioned responding (e.g., conditioned taste aversion) are extremely resistant to extinction. Furthermone, the observation that psychophysiological cue reactivity reflects general arousal responding can also be attributed to the fact that the measures in the present study are particularly sensitive to arousal. It is possible that other measures are more drug- 
specific and thus may have yielded a different pattern of results. For example, the alcoholdependent patients in group CERP often reported having a dry mouth and throat during exposure, whereas some of the cocaine dlependent patients specifically reported having a running nose when being exposed to cocaine-related cues. Treatment providers reported that these specific psychophysiological responses did tend to extinguish.

Nonetheless, the present results do demonstrate that cue-elicited arousal responding does not underlie or contribute to cue-elicited craving. Tiffany (1990) explicitly regards cue-elicted arousal and urge responding as independent measures and the present results thus appear to corroborate the view that psychophysiological cue reactivity in substance dependent persons reflects mental stress of not being able to automatically use a given drug or alcohol, as was suggested by Carter and Tiffany (1999). However, as according to Tiffany's cognitive model of addiction such mental stress should also give rise to drug urges, this notion too fails to explain the absence of a positive correlation between arousal responding and the subjectively experienced degree of craving. Further, this cognitive model cannot explain the apparent extinction of drug and alcohol craving in the present study. Although this then suggests that at least subjective cue reactivity reflects conditioned drug responding, an associative model of addiction cannot explain the present extinction of urges in both the group having received CERP and the group having received RT. The latter treatment is not designed to explicitly affect cue-elicited urges, whereas CERP is. This then is a somewhat puzzling result. However, again this is not an isolated result. Dawe and colleagues (1993), investigating the efficacy of cue exposure in the treatment of opiate addiction, found that both a group of patients receiving at least 6 individual cue exposure sessions and a group of patients receiving a control treatment (i.e., group therapy sessions) demonstrated a significant decrease in cue reactivity. As in the present study, these treatments were superimposed on a standard hospital treatment program. Dawe and colleagues argued that this standard treatment may contain elements of cue exposure treatment hence explaining the significant dlecrease in both groups. This is also true for the standard treatment program that the patients in the present study received at their treatment facility. One element of this program comprised discussing how to cope with high-risk drug-related situations. Although this is a much less structured and probably less intense form of exposure than a CERP session, it may have been sufficient for the extinction of cue-elicited craving. Of course, the extinction of urge responding in both groups can also be attributed to social desirable responding, that is, patients are unwilling to admit experiencing a strong urge to drink alcohol or to use a particular drug when still in treatment. Although such social desirable responding (or non-responding) cannot be discarded as a viable explanation for the present pattern of results, one then would have expected patients to also not demonstrate a clear pattern of cue-elicited craving at the cue reactivity assessment at pretest. Clearly, though, they did. 
The apparent survival of cue-elicited arousal as opposed to the decrease in cueelicited craving may be attributed to the fact that although one may very well learn to control drug urges, this still does not mean that one should become indifferent to being exposed to drug-related cues. Cues may obtain an affective value through evaluative conditioning. When a neutral stimulus is paired with a positive or negative cue, some of the valence of the affective stimulus will transfer to the neutral stimulus. In contrast to Pavlovian conditioned responding, this acquired valence is extremely resistant to an extinction procedure (see De Houwer, Thomas, \& Baeyens, 2001). The question is what valence drugrelated cues acquire. At face value, one could argue that drug cues should have a positive hedonic value as they refer to the positive rewarding effects of drug consumption. However, drug use also entails negative consequences. Indeed, as Robinson and Berridge (2000) have pointed out, many drug dependent patients can experience a strong desire to use a given drug and at the same time report disliking the drug.

The present discussion is admittedly speculative. Furdher research is thus warranted. Prominent research topics may concern determining the exact nature of cue-elicited arousal responding and the question to what degree such responding predicts frequency of drug consumption or the probability of a relapse. 
EXCESSIVE APPETTE 


\title{
CHAPTER 3
}

\section{THE EFFECT OF CUE EXPOSURE, URGE TO SMOKE, AND NICOTINE DEPRIVATION ON COGNITIVE PERFORMANCE IN SMOKERS*}

\begin{abstract}
The primary aim of this investigation was to test the hypothesis that the urge to smoke interferes directly with cognitive performance. Fifty-four smokers were randomly assigned to 1 of 3 groups: (i) Ad Lib, (ii) Deprived, or (iii) Nicotine Patch. Participants rated their urge to smoke on continuous Visual Analogue Scales. Cognitive performance was determined by measuring reaction times (RT) on a Sternberg task. The deprived group reported a higher urge and had longer RTs than the Ad Lib group when exposed to smoking related cues. However, the Nicotine Patclh group reported a higher urge in the absence of longer RTS. The results indicated that nicotine deprivation affects cognitive performance and that the urge to smoke only partially mediated RT's.
\end{abstract}

"This chapter is an adapted version of: Havermans, R.C., Debaere, S., Smulders, F.T.Y., Wiers, R.W., \& Jansen, A. (2003). Effect of cue exposure, urge to smoke, and nicotine deprivation on cognitive performance in smokers. Psychology of Addictive Behaviors, 17, 336-339. 
In 1990, Tiffany proposed a cognitiwe model of drug urges and drug use. According to this infliuential model of addictive behaviour, drug use behaviour becomes automatic due to repeated and frequent self-administration of drugs. The automatic drug use pattern is represented in long-term memory as an action schema, and its execution can be triggered by any cue related to drug use. Automatic drug use behaviour can be performed with relative ease and speed, and requires little cognitive effort. According to the model, the urge to use drugs only occurs when execution of the automatic drug use action schema is blocked. Impeding the execution of the drug action schema is established in two different ways. First, some external factor might prevent an addict to self-administer drugs, thus blocking the execution of the action schema. To nevertheless ensure the execution of the action schema, one has to address non-automatic cognitive processes. The use of these cognitive resources is reflected by the urge to use drugs. Tiffany calls this situation the abstinence-avoidance situation. Second, the execution of the drug-action schema can be blocked in an abstinence-maintenance situation. When an addict tries to remain drug-abstinent, he has to address non-automatic cognitive processes to block, or prevent the mindless execution of the drug action schema.

The assumption that urges reflect effortful cognitive processing can be tested by making use of a dual task procedure. In a typical dual task situation, performance on a particular cognitive task is impaired when one has to perform an additional cognitive task (Rah., Reber, \& Hsiao, 2000). If urges reflect the use of cognitive resources, then the urge to use drugs should interfere with performance on a cognitive task. The urge to use drugs is thus expected to disrupt any kind of activity requiring cognitive resources (Tiffany, 1990). As Cepeda-Benito and Tiffany (1996) state: "if craving represents the operation of capacity-limited non-automatic processes, it should operate at the cost of disnupting other activities that also demand non-automatic processing" (p. 155).

Some empirical support for this hypothesis has been found. For instance, CepedaBenito and Tiffany (1996) studied the effect of the urge to smoke on a simple reaction time (RT) task. They induced the urge to smoke by having smokers memorise urge-eliciting sentences, which they were instructed to retrieve at a given signal while simultaneously performing a simple RT task. They found that the urge to smoke slows RT. Sayette and Hufford (1994) also studied a potentially detrimental effect of the urge to smoke on RT in two experiments. They manipulated the urge to smoke and found that urges interfere with performance on a simple RT task when smokers were abstinent and received exposure to smoking related cues. A positive correlation was found between the urge to smoke and RT in the first experiment, although this correlation failed to reach significance in the second experiment.

These investigations provide some support for the hypothesis that the urge to use drugs disnupts cognitive performance. However, these studies do not allow for a ubiquitous statement that the urge to use drugs is reflected by nonautomatic cognitive processing di- 
rectly interfering with performance on a cognitive task. It is conceivable that drug deprivation plays an important, if not a crucial role in tinding detrimental cognitive performance. Research on the psychopharmacological effects of nicotine for instance, has led to the general finding that nicotine delivery influences information processing (Spilich, June, \& Renner, 1992; Kassel, 1997; Rusted, Graupner, Tennant, \& Warburton, 1998).

The primary aim of the present study was to determine whether the urge to smoke interferes directly with performance on a cognitive task. This question was studied comparing cognitive performance between smokers being nicotine deprived and smokers allowed smoking ad libitum, using a Sternberg memory search task. To be able to determine whether the urge to smoke causes a detrimental effect on cognitive performance, a third group of smokers was instructed to apply a transdermal nicotine patch 12 hours before the onset of the experiment and to abstan from smoking during that period. It has been found that transdermal nicotine delivery has about the same effects on perfonmance (i.e., information processing) and mood as cigarette smoking (Warburton \& Mancuso, 1998). However, it has also been found that strong urges can still be elicited in smokers wearing a nicotine patch when they are exposed to smoking related cues (Tiffany, Cox, \& Elash, 2000). This experimental group thus enables to determine whether the urge to smoke affects cognitive performance independent from a psychopharmacological effect. If the urge to smoke demands cognitive processes, performance on the Sternberg task should be disrupted in these participants. If, however, nicotine deprivation plays an important role in disrupting cognitive performance, participants in this group should not show a performance deficit.

The secondary aim of this study was to determine whether the urge to smoke specifically disrupts performance on the Sternberg memory search task when the task requires sustained attention. An effect of the urge to drink alcohol on RT was found by Sayette, et al. (1994) in recovering alcoholics. In vivo exposure to an alcoholic beverage produced a large detrimental effect on $\mathrm{RT}$, whereas exposure to a glass of water did not produce such an effect. The intervals between the RT trials were variable in this particular study and thus required sustained attention. When intervals between trials are relatively short and fixed, ans was the case with the RT task employed by Cepeda-Benito and Tiffany, one easily learns to predict the onset of the target stimulus, leading to response preparedness (Nieni \& Näătänen, 1981). The extremely large effect of the urge to drink on RT found by Sayette, et al., as opposed to the more modest effect of the urge to smoke on RT reported by Cepeda-Benito and Tiffany, suggests that addicts particularly show detrimental cognitive performance on a task when this task requires sustained attention.

The Sternberg task is a choice-RT task requiring scanning through working memory (Stemberg, 1966). A memory set containing a given number of items is presented and participants are instructed to memorise this set. Next, target-items are presented and now one has to decide whether the presemted target is a member of the memory set or not. One half of the presented target-items belongs to the memory set and the other half of the presented. 
target-items does not belong to the memory set. Typically, a linear relation is found between the size of the menory set and RT. The general explanation for this linear relationship is that one performs an exhaustive search through the memory set in working memory. Thus, the larger the memory set, the longer the search. The advantage of the Sternberg task is that it not only gives an estimate of the sum of the duration of basic perceptual and motor processes, comparable to simple RT tasks, but also an estimate of the isolated duration of a purely mental process, namely capacity demanding memory search (Sternberg, 1966, 1975). Therefore, the Sternberg task may be a more sensitive measure of the disrupting effects of urges on cognitive performance than a simple RT task.

To study the question whether sustained attention is particullarly impaired in smokers experiencing urge, the Sternberg memory search task used in the present study was modified. A warning signal preceded the presentation of the target-item. In one condition the interval between warning signal and target-item was varied per trial and in the other condition the interval was fixed. In the variable interval condition, one cannot predict the onset of the target-item and thus the task requires sustained attention (see Niemi \& Näätänen, 1981; Gottsdanker, Perkins, \& Aftab, 1986).

In sum, the questions of interest are whether the urge to smoke interferes directly with performance on a Sternberg memory search task, and whether the urge to smoke particularly disrupts performance when the task requires sustained attention.

\section{Method}

\section{Participants}

Fifty-four smokers ( 22 men and 32 women) were recruited by posted advertisements. All participants were at least 18 years ofd $(M=22,6, S D=3.8$, range $=18-42)$ and smoked at least 10 cigarettes a day $(M=18.5, S D=4.8$, range $=10-35)$. Chronic physical or mental illness, and/or taking any medication affecting cognitive performance at the time of the experiment were used as the exclusion criteria. Each participant was paid fl. 20 (the approximate equivalent of $f 9$ ) on completion of the experiment. Participants" characteristics are shown in Table 3.1.

\section{Level of dependence}

Ulsing the Fagerström Test of Nicotine Dependence (FTND) (Heatherton, Kozlowski, Frocker, \& Fagerström, 1991), the level of nicotine dependence for each participant was assessed. This is a self-report measure containing 6 items concerning smoking behaviour. The score on this test ranges from 0 (not nicotine dependent) to 10 (highly nicotine dependent). 
Table 3.1

Number of participants, mean number of consumed cigarettes per day, mean FTND-Scores, and mean CO-level for each group.

\begin{tabular}{l|rrr}
\hline \multirow{2}{*}{ Variables } & \multicolumn{3}{c}{ Group } \\
\cline { 2 - 4 } & \multicolumn{1}{|c}{ Ad Lib } & \multicolumn{1}{c}{ Deprived } & Nicotine Patch \\
\hline$N$ & 18 & 18 & 18 \\
Cig/day & $17.5(4.6)$ & $18.1(4.1)$ & $19.8(5.7)$ \\
FTND-score & $3.6(2.1)$ & $4.6(2.0)$ & $4.5(2.0)$ \\
CO-level (ppm) & $16.2(6.9)$ & $11.1(1.6)$ & $11.1(3.0)$ \\
\hline
\end{tabular}

Note. Vallues enclosed in parentheses represent $S D$.

\section{Urge to smoke}

Participants were randomly assigned to one of three groups: a group of active, ad libitum smokers (Ad Lib), or the group of abstinent smokers receiving cue exposure with response prevention to smoking-related cues (Deprived), or the group containing smokers wearing a transdermal nicotine patch receiving cue exposure with response prevention (Nicotine Patch). All participants were instructed to bring their own pack of cigarettes to the experiment. Participants in group Ad Lib were instructed to smoke ad libirum before the onset of the experiment and to snoke a cigarette prior to the experimental task. Participants in groups. Deprived and Nicotine Patch were instructed to abstain from smoking for 12 hours before the start of the experiment. The participants in group Nicotine Patch wore a. $21 \mathrm{mg}$ transdermal nicotine patch (Nicotinell TTS 30, Lohmann Therapie Systeme) during the 12-hour period of smoking abstinence. It was expected that the smoking abstinent participants (group Deprived and Nicotine Patch) would experience a high level of urge whereas the ad ibitum smokers (group Ad Lib) would experience no (or hardly any) urge to smoke.

The urge to smoke was measured using single-item 100-min Visual Analogue Scales (VASs) on which the participants had to rate their urge to smoke at that moment, scores ranging from 0 ("no urge to smoke at all") to 100 ("a near irresistible urge to smoke"). Although the use of singleitem measures of smoking urges has been criticised, Kozlowski et al. (1996) argue that such measures may not necessarily be as deficient as has been suggested. More complex, multiflactorial questionnaires may even obscure the measurement of smoking triges.

\section{Smoking abstinence}

To be able to check whether the participants from the two smoking-abstinent groups (Deprived and Nicotine Patch) had complied to the abstinence instructions, a sample of end-expired air was taken from each participant and subsequently analyzed, using a Miran $1 \mathrm{~B}$ infrared gas analyzer (FOXBORO, East Bridgewater, MA), on the content of carbon monoxide (CO). 


\section{Cognitive performance}

Performance on the Sternberg memory search task was measured using an IBM-compatible computer with msec-timing capabilitits. A fixed set Stemberg memory search task was used in this experiment $A$ memory set containing 2,4 , or 6 digits was presented before each block of trials. The digits in the memory set were drawn random without replacement before each block of trials by the computer. Participants were instructed to memorise the presented set. One second after participants had indicated that they had memorised the set, a warning signal was given to signal the upcoming presentation of a target-digit. The waming signal consisted of a 500 msec computer generated tone and the simultaneous increase in size of the edges of a square continuously present at the centre of the screen during the task. The target-digits were presented within this square.

The time-imterval between warning sugnal and probe is called a foreperiod, In the fixed foreperiod condition, the interval between the warning signal and the probe had a fixed duration of 2.5 sec. In the variable foreperiod, this interval was waried randomly per trial around a mean of $2.5 \mathrm{sec}$ and was nonaging, which ensures that the timing of the presentation of the target-digit cannot be predicted by the participant (see Gottsdanker, et al. 1986).

The participant's task was to decide whether the shown target-digit belongs to the memory set. Participants responded by pushing either a 'yes'-key to indicate that the presented digit belongs to the memory set (a positive response), or by pushing a "no'-key as to indicate that the presented digit does not belong to the memory set (a negatiwe response). Half of all participants in each group had the "yes"-key on the left and the "no"-key on the right of the response pad, and the other half had the opposite assignment. Participants were instructed to keep their index fingers on the response keys at all times during the task.

Each participant received a total of 144 trials, equally divided over 6 different blocks of 24 trials. The blocks differed in memory set size and foreperiod and the order of presentation of the blocks was counterbalanced by a latin square, rendering 6 different orders of blocks. Counterbalancing of the order of presentation of blocks controls for potential order effects (e.g., practice or fatigue).

\section{Design}

A mixed factorial design was used with Group (Ad Lib, Deprived, or Nicotine Patch) as between-subjects factor and Response (positive, or negative), Set size $(2,4$, or 6$)$, and Foreperiod (fixed, or variable) as within-subject factors..

\section{Procedure}

All participants were given a brief description of the experimental procedure and signed a consent form before participating in the experiment. They were instructed to abstain from any product containing alcohol or caffeine for at least 12 hours before the start of the experiment. Parlicipants assigned to the group Nicotine Patch were instructed to apply a transdermal nicotine patch 12 hours before serving in the experiment and to abstain from smoking during that period. All experimental sessions were conducted in the morning between 9 and $11 \mathrm{AM}$.

At arriwal, a sample of end-expired air was taken from each participant and analysed on the content of carbon monoxide (CO). Next, the participant was led to a quiet research room. In this room each participant was asked to rate his/her urge to smoke at that moment (urge measurement 
1). The experimenter read the instructions of the Stemberg task and the task was practiced. Bach of the 6 different blocks of trials was practiced for I min with the experimenter monitoring performance. After this practice session, participants in the two smoking abstinent groups (Deprived and Nicotine Patch) were exposed to smoking cues. This cue exposure with response prevention (CERP) consisted of handling a cigarette of their favourte brand, to simulate snoking with this cigarette as well as simulate smoking the cigarette when lit. This cue exposure session had an approximate duration of $6 \mathrm{~min}$. Participants in group Ad Lib were imstructed to smoke a cigarette. After simulated or actual smoking, the participants had to rate their urge to smoke again (urge measurement 2), after which the experimental Sternberg task was run. During this task, the experimenter sat within the left peripheral view of each participant and smoked cigarettes while participants trom the two smoking abstinent groups (Deprived and Nicotine Patch) were performing the Sternberg task. To control for his presence and movements, the experimenter played with a pen, occasionally taking notes, while participants from group Ad Lib were performing the Sternberg task. After each block, participants rated their urge to smoke on a VAS (urge measurements 3 through 8 ).

\section{Results}

\section{Manipulation checks}

Figure 3.1 depicts the mean level of self-reported urge per group as rated by the participants on a VAS throughout the experiment. To check whether the abstinence instruction led to differences between the groups on self-reported urge at baseline, a one-way ANOVA was performed with Group (Ad Lib, Deprived, or Nicotine Patch) as betweensubjects factor and the urge-score at baseline (urge measurement 1) as the dependent variable. A difference in urge-scores was found between the groups, $F(2,51)=5.4, p<.01$. Subsequent contrast-analyses, using a Bonferroni corrected rejection criterion of $2.5 \%$, revealed a difference in urge between the active smokers (Ad Lib) and the two smokingabstinent groups (Deprived and Nicotine Patch) taken together, $r(52)=2.8, p<.01$. As expected, the active, ad libinm smokers showed less urge to smoke than the abstinent smokers. No difference in urge was found between the two smoking abstinent groups (Nicotine Patch vs. Deprived), $t(34)=1.7, \mathrm{~ms}$. 


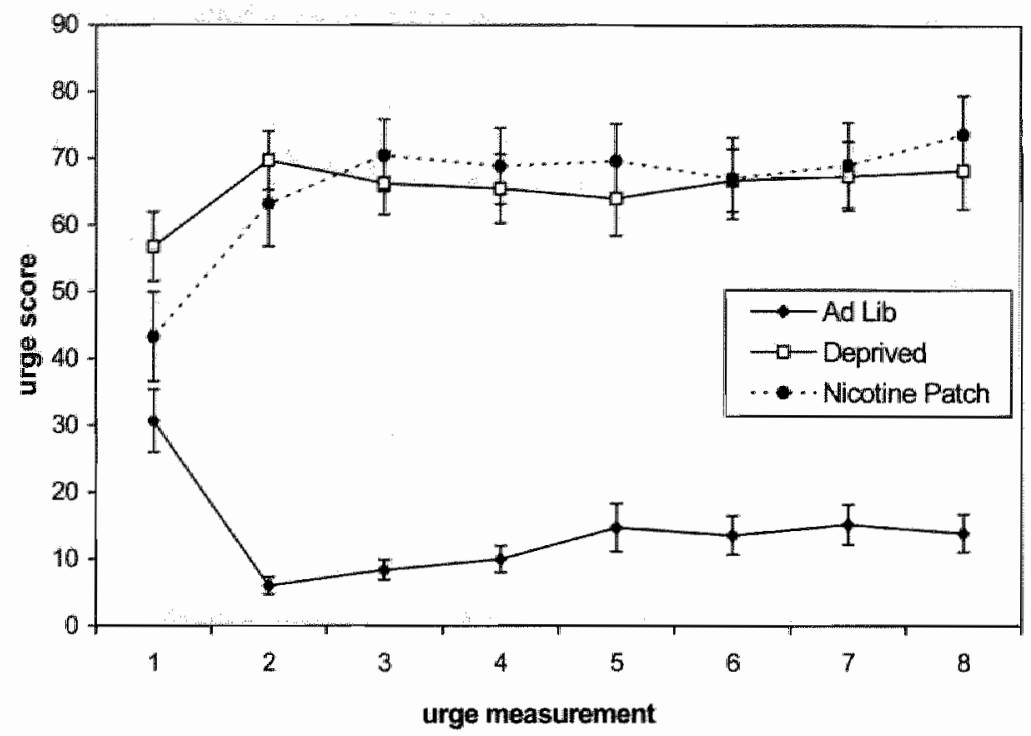

Figure 3.1. Mean level of self-reported urge ( \pm SEM) per urge measurement and for each group (Ad Lib, Deprivation, and Nicotine Patchi, as rated on visulal analogue scales throughout the experiment.

A similar one-way ANOVA on the urge-scores measured after smoking or exposure also revealled a significant difference in urge between groups, $F(2,51)=59.2, p<.001$. Contrast-analyses revealed a difference in urge comparing the ad libitum smokers (Ad Lib) with the two abstinent groups taken together (Deprived plus Nicotine Patch), $t(52)=10.8$, $p<.001$. The abstinent smokers reported larger urge ratings after being exposed to smoking cues than the active smokers who had to smoke a cigarette. No difference in urge was found between the two abstinent groups (Deprived vs. Nicotine Patch), $t(34)=1.0$, ns. These results clearly demonstrate that, in accordance with results reported by Tiffany, et al. (2000), cue-elicited urges can be observed in smokers wearing a nicotine patch.

A two-way ANOVA was performed with Group (Ad Lib, Deprived, or Nicotine Patch) as between-subjects factor and Time (urge measurements 3 through 8 ) as the within-subject variable, with the mean urge score per rating measured after each block of trials during performance on the Sternberg task. A difference in urge scores between groups was found, $F(2,51)=50.4, p<.001$. Contrast-analyses showed a difference in urge between group Ad Lib and the two smoking-abstinent groups taken together (Deprived plus Nicotine Patch), $(52)=10.0, p<.001$. Throughout the experiment, the abstinent smokers reported a larger urge to smoke than the ad libinum smokers. No difference in the extent of urge was found between group Deprived and group Nicotine Patch, $t(34)=0.5$, $n s$. These results make clear that there was no difference in the extent of reported urge be- 
tween the nicotine-deprived smokers and the smokers wearing a nicotine patch. No main effect of Time was found, $F(5,255)=2.0, n s$. This result indicates that the level of reported urge to smoke did not fluctuate during performance on the Stemberg memory search task. No interaction between Group and Time was found, $F(10,255)=1.4$, ns.

To check whether participants from group Deprived and group Nicotine Patch had complied with the given instruction to abstain from smoking, each of these participants was asked when they had last smoked a cigarette. This was asked after the experimental session and after payment. None of the participants reported non-compliance. Further, participants' carbon monoxide (CO) levels were assessed prior to the experiment. Typically, smoking abstinence leads to a reduction in the level of $\mathrm{CO}$ in end-expired air. Thus, the active smokers should have higher levels of $\mathrm{CO}$ than the abstinent smokers should.

A one-way ANOVA with Group (Ad Lib, Deprived, or Nicotine Patch) as the between-subjects factor and level of $\mathrm{CO}$ measured in end-expired air as the dependent variable, showed a significant difference in the level of CO between groups, $F(2,51)=7.9, p<$ 01. Contrast-analyses were conducted and these revealed a significant difference in the level of $\mathrm{CO}$ between the two smoking abstinent groups (Deprived and Nicotine Patch) taken together in comparison with the ad libitum smokers (group Ad Lib), $r(52)=4.0, p<$ .001 . The level of $\mathrm{CO}$ was lower in the two smoking abstinence groups compared to the level of $\mathrm{CO}$ in the active smokers. No difference in the level of $\mathrm{CO}$ was found comparing group Deprived with group Nicotine Patch, $t(34)=0.1$, $n s$, which indicates that the level of $\mathrm{CO}$ did not differ between the two smoking abstinent groups as one would expect. However, it should be noted that the mean level of end-expired $\mathrm{CO}$ was still relatively high in both smoking abstinent groups, whereas the mean level of $\mathrm{CO}$ in the ad libitum smokers was relatively low. The primary reason for this finding is that testing took place early in the morning. Some participants in the Ad Lib group indicated that they had not smoked a cigarette yet at the time of the breath-analysis. These participants were thus over night abstinent, explaining the relatively low mean level of $\mathrm{CO}$ for this group. As $\mathrm{CO}$ is expelled more slowly over night, the mean level of $\mathrm{CO}$ was still relatively high in both abstinence groups. Nevertheless, $\mathrm{CO}$ lewels in a few participants were exceptionally high (i.e., more than $1 S D$ above the mean level of $\mathrm{CO}$ in their respective groups); 1 participant from group Deprivation and 1 participant from group Nicotine Patch had a CO level of $15 \mathrm{ppm}$, and 2 participants of group Nicotine patch had a CO level of $17 \mathrm{ppm}$. Since it is highly unlikely that these participants complied with the smoking abstinence jnstruction, data from these participants were excluded from the following analyses. 
The influence of urge and nicotine deprivation on cognitive performance

RT latencies of the responses on the Sternberg task exceeding $2.5 S D$ from average were considered probable outliers and were removed (see Ratcliff, 1993). A one-way ANOVA with Group (Ad Lib, Deprived, or Nicotine Patch) as the between-subjects factor and number of errors as the dependent variable rewealled no difference in the number of errors between the groups, $F(2,45)<1$. The overall percentage of errors equalled $3.8 \%$. According to Stemberg (1975), a percentage of errors below $10 \%$ does not affect the linear relationship between memory set size and RT representing the memory scanning process.

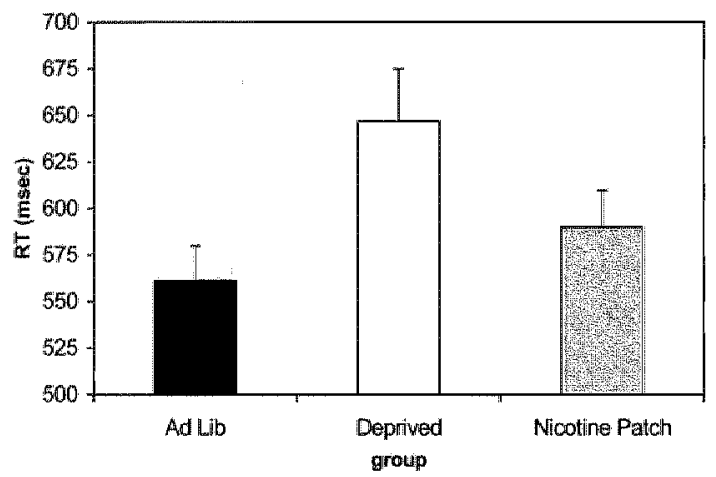

Figure 3.2. Mean reaction time in msec of the participants' responses on the Sternberg memory search task (+ SEM) for each group (Ad Lib, Deprivation, an Nicotine Patch).

Figure 3.2 depicts the mean RT in msec of the responses on the Sternberg task plus the standard error of the mean (SEM) for each group. A four-way ANOVA was performed with Group (Ad Lib, Deprived, or Nicotine Patch) as between-subjects factor, and Response (positive, or negative), Set size $(2,4$, or 6 ) and Foreperiod (fixed, or variable) as within-subject factors with $\mathrm{RT}$ in msec of the correct responses as the dependent variable.

A main effect of Response was found, $F(1,47)=10.2, p<.01$. Positive responses (i.e., a response made to indicate that a target item is a member of the memory set) are faster than negative responses. This result is in agreement with the general findings regarding the Sternberg task (see Stemberg, 1975). A main effect of Set size was found, $F(2,94$ ) $=118.2, p<.001$. This result reflects the increase in $\mathrm{RT}$ when the memory set is enlarged. A main effect of Foreperiod was found $F(1,47)=6.4, p<.05$, indicating that RT was larger when the foreperiod was variable. A main effect of Group was found, $F(2,47)=3.9, p$ $<.05$, indicating that there is a difference in RT latency between the groups. No interaction effects were found, $p$ 's $>.10$.

Contrast-analyses revealed a significant difference in overall RT between group Deprived and the non-deprived participants from group Ad Lib plus Nicotine Patch, $t(48)=$ $2.6, p<.025$, effect size $d=0.8$. No difference in RT was found between group Ad Lib 
and the participants from group Nicotine Patch plus Deprived, $t(48)=2.1$, ns, effect size $d$ $=0.6$.

The results appear to suggest that nicotine deprivation, rather than urge, particularly interferes with cognitive performance. To test the hypothesis that the effect of nicotine deprivation on RT is mediated by the subjective urge to smoke, a mediation analysis was conducted as specified by Baron and Kenny (1986). First, the unmediated model, which tests the effect of nicotine deprivation on RT without controlling for the urge to smoke, was tested. Participants were recoded as being nicotine deprived (participants from group Deprived) or not (participants from group Ad Lib plus Nicotine Patch). It was found that nicotine deprivation (yes, or no) predicts RT latency, $\beta=.36, F(1,48)=6.9, p<.05$. Nicotine deprived participants show slower response times than the non-deprived participants.

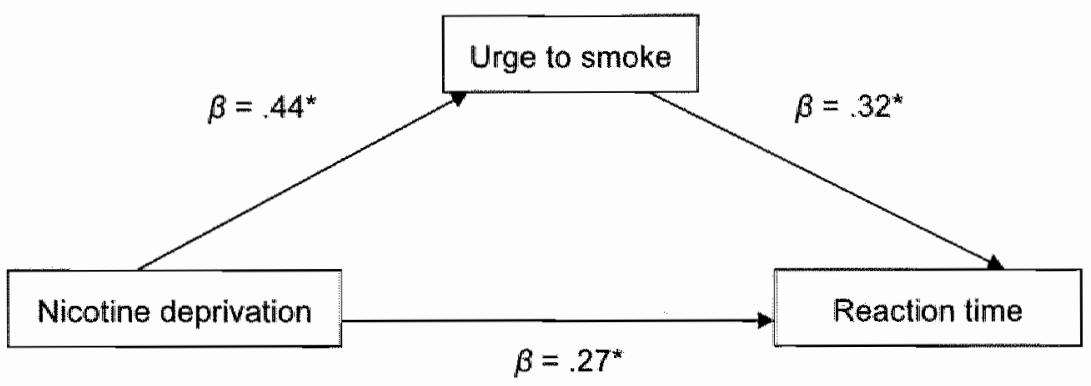

Figure 3.3. Mediation model of the effect of nicotine deprivation on RT, controlled for the effect of urge to smoke. ${ }^{*} p<.05$.

Next, the mediated model, controlling for a potential effect of the urge to smoke on cognitive performance, was tested. The mediated model is displayed in Figure 3.3. It was found that the wariable nicotine deprivation predicts the level of experienced urge to smoke, $\beta=.44, F(1,48)=11.2, p<.01$. The nicotine-deprived participants reported a higher level of the urge to smoke than the non-deprived participants taken together. The urge to smoke predicted $\mathbb{R T}, \beta={ }_{3} 32, F(1,48)=5.3, p<.05$. Increase in the urge to smoke is accompanied by an increase in RT. However, the effect of nicotine deprivation on RT remains statistically significant, $\beta=.27, F(1,48)=4.4, p<.05$. The level of significance, however, decreases when one controls for the effect of the urge to smoke on RT suggesting that the urge to smoke partially mediates the effect of nicotine deprivation on RT. It should be noted, though, that the urge to smoke accounts for only $25 \%$ of the effect of nicotine deprivation on $\mathrm{RT}$. 


\section{Discussion}

The primary aim of the present investigation was to test Tiffany"s hypothesis (1990) that the urge to smoke directly interferes with performance on a cognitive task. The results of the present study do not support this hypothesis. As long as nicotine is present, the urge to smoke thardly interferes with performance on the Sternberg memory search task, suggesting that the urge to smoke in itself is not sufficient to interfere with cognitive performance as Tiffany $(1990,1997)$ proposes. This finding is in agreement with several recent studies reporting the absence of a strong relation between the urge to smoke and cognitive performance (see Juliano \& Brandon, 1998; Baxter \& Hinson, 2001).

The secondary hypothesis of the present study was that a task requiring sustained attention is particularly troublesome for smokers experiencing urge. No support for this hypothesis was found. All the participants, whether they experienced the urge to smoke or not, showed slowing of RT in the variable foreperiod condition, which is in line with the more general notion that a task requiring sustained attention is considered more difficult than a task which does not require sustained attention. Therefore, why Sayette, et al. (1994) found such a large detrimental effect of the urge to drink on RT in alcoholics, as opposed to the more modest effect of the urge to smoke on RT reported by Cepeda-Benito and Tiffany (1996) cannot be attributed to a potentiall difference in response preparedness between these two studies.

An additional finding is that cue-elicited urge to smoke can still occur in smokers wearing a nicotine patch, which is in agreement with Tiffany, et al. (2000). Tiffany, et al. also found that wearing a nicotine patch attenuated abstinence induced cigarette craving. No effect of the transdermal nicotine patch on abstinence induced urge to smoke was found in the present investigation. However, participants wearing the nicotine patch might have been exposed to smoking related cues before their participation in the experiment, thus exhibiting relatively high levels of self-reported urge to smoke at baseline. This finding then provides an account of the observation that some individuals continue smoking despite wearing a transdermal nicotine patch (see Foulds, et al., 1992). Furthermore, cue elicited urge does not seem to depend on the amount of abstinence induced urge to use a given drug as Drummond (2000) recently suggested.

The present results thus indicate that the observed differences in RT between the groups can be primarily ascribed to differences in nicotine deprivation. This suggests a purely psychopharmacological effect. However, an alternative explanation for the observed differences in RT is to state that the urge to smoke affects RT indirectly. That is, the urge to smoke only affects cognitive performance when one is nicotine deprived. This notion is in agreement with earlier findings by Sayette and Hufford (1994). They found no effect of the urge to smoke on RT in participants exposed to smoking related cues, and found no effect of the urge to smoke on RT in smokers deprived from nicotine (although these ma- 


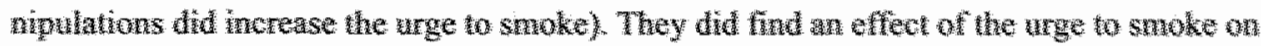

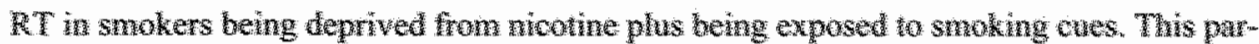

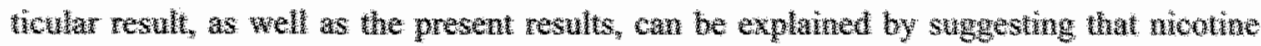

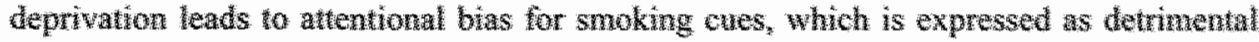

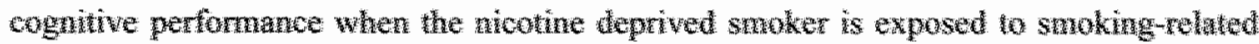

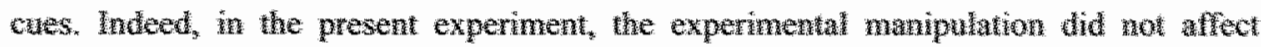

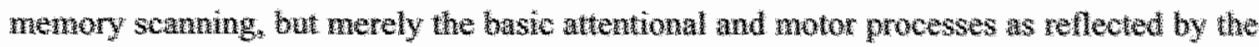

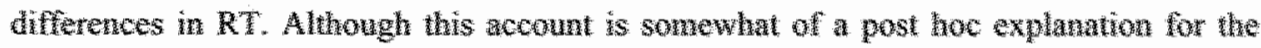

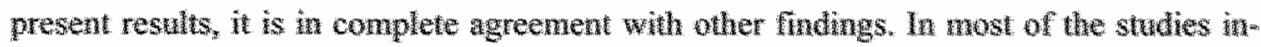

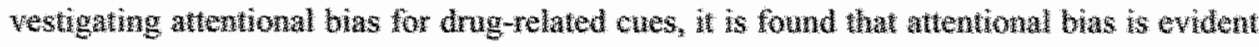

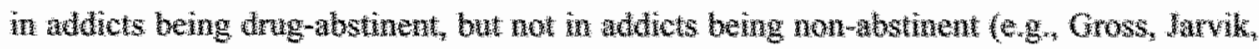

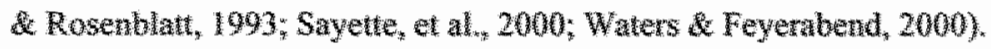

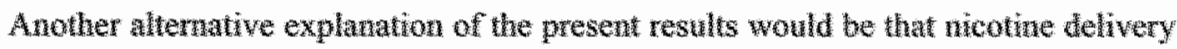

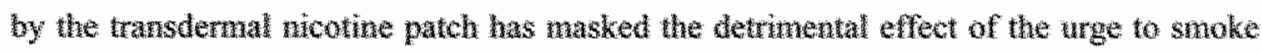

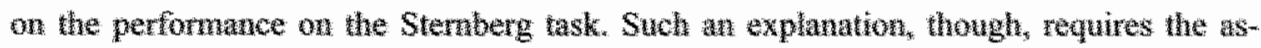

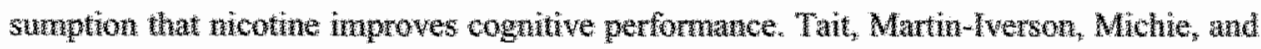

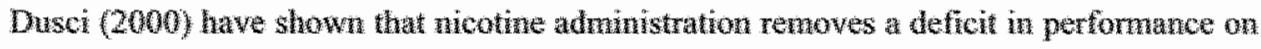

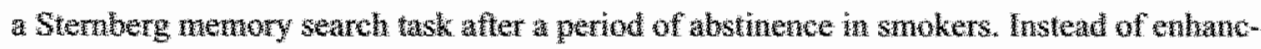

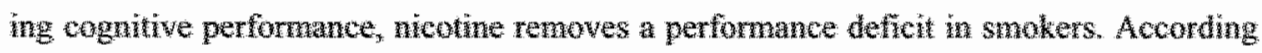

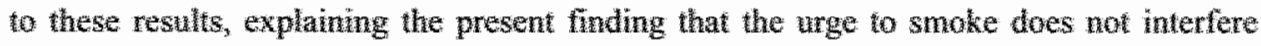

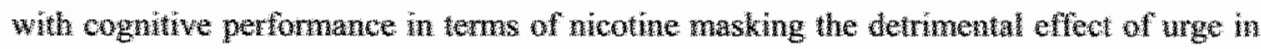
smokers wearing a ncotine patch w whorobabit.

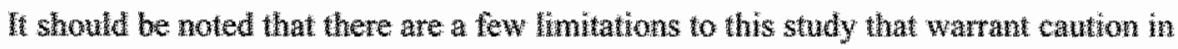

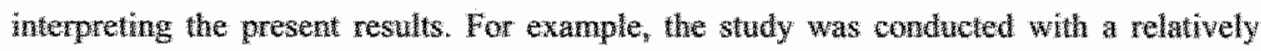
smant sample size. "The absence of a clear eflect of the whe somoke on cogntive percomance could be ascrbed to ack of power. The present sample size alows for the detection of large effects only. The detection of a small to medium effect would have required the recruitment of far more participants (see Cohen, 1992).

In sum, it was found that the urge to smoke merely partially mediates performance on a cognitive task requiring attentional, psychomotor and memory processes. Further, the urge to smoke only appeared to have a small and nonspecific effect on $\mathrm{RT}$, suggesting that not all cognitive processing is affected when experiencing the urge to smoke. 
ExCESGive APYLTrE 


\title{
CHAPTER 4
}

\section{INCREASING THE EFFICACY OF CUE EXPOSURE TREATMENT IN PREVENTING RELAPSE OF ADDICTIVE BEHAVIOUR*}

\begin{abstract}
Theoretically, cue exposure treatment should be able to prewent relapse by extinguishing condi tioned drug responding (e.g., cue-elicited craving). According to contemporary learning theory, though, extinction does not eliminate conditioned responding. Analogous, cue exposure with response prevention as a treatment of addictive behaviour might not eliminate the learned relation between drug related cues and drug use. This does not necessarily mean that cue exposure cannot successfully prevent relapse. Various suggestions for increasing the efficacy of cue ex* posure treatment are being discussed from a contemporary leaming theory perspective. It is suggested that cue exposure treatment incorporating retricval cues can be a benellicial treathient in preventing relapse of addictive behaviour.
\end{abstract}

\footnotetext{
"This chapter is an adapted version of: Havermans, R.C., dansen, A. (2003). Increasing the efficacy of cue exposure treatment in preventing relapse of addictive behavior. Addicfive Behaviors, 28, 989-994.
} 
Cue exposure with response prevention (CERP) refers to the treatment in which a drug addict is repeatedly exposed to stimuli associated with their addictive behaviour. These drug related stimuli are termed cues and the reactivity these drug cues elicit is generally considered to be a conditioned drug response. The conditioned drug responses can be subjective (e.g., craving), psychophysiological (e.g., changes in heart rate), or behavioural (e.g., drug seeking behaviour) and these responses are considered to motivate and/or increase the likelihood of drug self-administration. Cues thus function as conditioned drug stimuli predicting drug taking behaviour. Repeated exposure to these drug rellated cues preventing selfadministration of the drug should eliminate the learned relation between drug cues and drug use. This treatment leads to the gradual extinction of the conditioned drug responses. It has been suggested that this form of treatment might limit the probability of relapse. When these responses are extinguished the primary motivation for continued drug use is eliminated and thus the probability of relapse of addictive behaviour should be small (Drummond, et al., 1995; Jansen, 1998).

Several controlled trials investigating the efficacy of cue exposure treatment have been conducted. None of these studies found a substantial effect on relapse by extinguishing cue reactivity. Dawe, et al. (1993) studied the efficacy of cue exposure treatment in opiate addicts and found that cue exposure had no additional effect on relapse rates as compared with a control group of patients receiving a standard inpatient treatment. Drummond and Glautier (1994) did find an effect of cue exposure treatment on relapse in alcoholics. They found that cue exposure treatment increased the latency to relapse, though the relapse rate did not differ from a control group of patients having received relaxation training. Niaura, et al. (1999) studied the efficacy of cue exposure treatment in smokers for smoking relapse prevention and found that it did not affect relapse at all. O'Brien, et al. (1990), investigating the efficacy of cue exposure treatment in recovering drug dependent patients, found that cue reactivity (i.e., craving) as well as occasional drug use still occurred outside the treatment setting, even in those patients in which conditioned drug responding had been successfully extinguished.

Apparently, the extinction of cue reactivity does not generalise beyond the treatment setting. How can this be explained? Extinction is often regarded as the "unlearning" of a previously learned behaviour. This view of extinction, however, implies that extinction is forever; unfortunately it is not (see Bouton, 1994). Conditioned responding can recover after extinction, which indicates that extinction is not merely some form of "unlearning". This fact leads to the prediction that CERP in its present form might be inadequate in eliminating conditioned drug responding (i.e., cue reactivity) and thus in limiting the probability of relapse. Cue exposure leads to the extinction of cue reactivity, but cue reactivity may recover after treatment. The notion of recovery of conditioned drug responding can explain why cue exposure treatment has not been found to be a successful technique in limiting relapse of addictive behaviour. If somehow the recovery of extinguished cue reac- 
tivity could be prevented, cue exposure treatment might be additionally beneficial in pre. venting relapse of addictive behaviour after all.

In this chapter, several suggestions to improve the efficacy of CERP as a treatment of addictive behaviour are discussed. The described issues all follow from contemporary research on learning and motivation, and are reformulated for clinical practice. First, it is described how contemporary learning theory defines extinction and how it explains the recovery of conditioned responding. Second, different methods to prevent the recovery of extinguished cue reactivity are discussed. Suggestions for future research are addressed.

\section{Theoretical Notes}

\section{Recovery of conditioned responding}

Pavlov (1927) already noted that extinction is not forever. He found that extinguished conditioned responding can recover some time after extinction. This effect has been termed spontaneous recovery and it clearly demonstrates that extinction does not eliminate or break the initially learned relation between a conditioned stimulus (e.g , a tone) and an unconditioned stimulus (e.g., food). Another phenomenon indicating that extinction is not the 'unlearning' of an association is the renewal effect. This effect refers to the renewal of conditioned responding after extinction when the conditioned stimulus is presented in an environmental context different from the context in which extinction has taken place. Bouton (1994) states that the conditioned stimulus has acquired an ambiguous meaning after extinction. After extinction, this stimulus predicts both the presentation as well as the absence of the unconditioned stimulus. In other words, the conditioned stimulus has acquired an excitatory meaning during conditioning versus an inhibitory meaning acquired during extinction. An organism can disambiguate the meaning of the conditioned stimulus by letting its inhibitory meaning depend on the environmental context. Within the extinction context, the conditioned stimulus has an inhibitory meaning, whereas outside this context, the conditioned stimulus has an excitatory meaning capable of eliciting conditioned responding. According to Bouton (1993), the inability of the generalisation of the inhibitory meaning of the conditioned stimulus outside the extinction context is due to the organism's inability to retrieve this meaning. Spontaneous recovery can also be explained in terms of the inability to retrieve the meaning of the conditioned stimulus in an environment other than the extinction context. Extinction takes place against a background of all kinds of stimuli, including time. When the conditioned stimulus is re-presented some time after extinction, conditioned responding is renewed because the conditioned stimulus is now presented in a temporal context different from the temporal context in which extinction has taken place. 
These findings pose serious implications for cue exposure treatment (Bouton, 1988). Extinction is not forever and thus it is quite conceivable that conditioned drug responding can be renewed afuer cue exposure treatment. The question here is how this potential recovery of conditioned drug responding can be prevented.

\section{Cue exposure within the drug use environment}

One does not need to worry whether the inhibitory meaning of a drug cue generalises to the context in which the addict used to take drugs when you conduct CERP in this particular environment. However, this is not always possible, and an additional problem is that addictive behaviour is not necessiarily restricted to one particular environment. Some addicts may administer their drugs in wide variety of settings and situations (Hammersley, 1992). Furthermore, this procedure would entail an outpatient treatment in which it is difficult to control for drug use between sessions. It is of great importance that a patient undergoing cue exposure treatment does not take any drugs during this treatment, since such a lapse might re-establish the originally learned relation between drug cues and drug use. In sum, although conducting cue exposure within the drug use environment can theoretically prevent renewal of cue reactivity, there are just too many practical limitations to be considered as the ideal alternative to 'standard' CERP.

\section{Cue exposure within multiple contexts}

In case it is impossible to conduct CERP in the drug use environment, one needs to achieve generalisation of the inhibitory meaning of the drug cues to contexts other than the treatment setting. An often proposed way of establishing this form of generalisation is to conduct CERP in mulltiple settings (see Gunther, Deniston, \& Miller, 1998). The general idea is that conducting CERP in a number of different environments enhances the probability of generalisation of extinguished cue reactivity to an environment other than the treatment setting, thus limiting the probability of renewed cue reactivity. In animall studies it has been shown that this procedure indeed reduces the probability of renewal. However, conducting CERP in multiple settings will slow down the extinction of cue reactivity. Furthermore, it is completely unclear in how many different contexts one needs to conduct CERP to prevent renewal of conditioned drug responding. Additionally, one can seriously doubt whether an addict in an inpatient treatment will perceive various, artificially created settings within one and the same treatment facility as distinct environmental contexts.

Even when these drawbacks can be overcome, this method does not prevent spontaneous recovery of drug cue reactivity. One way to tackle this problem is to re-administer CERP at intervals to reduce the probability of spontaneous recovery (Hammersley, 1992; Conklin \& Tiffany, 2002). This procedure, however, lengthens the treatment even further. The ideal CERP then would be a treatment that considers and controls for the probability 
of a renewal effect and spontaneous recovery without having to deal with the drawbacks mentioned above.

\section{Cue exposwre with retrieval cues}

Conducting CERP within multiple settings at intervals can establish the generalisation of extinguished cue reactivity, but has its obvious drawbacks. Another, more promising option is to conduct CERP incorporating retrieval cues. Retrieval cues are salient features of the extinction environment which facilitate retrieval of the inhibitory meaning of the conditioned stimulus when presented outside the extinction context. Bouton (1993) re: gards both the renewal effect as well as spontaneous recovery as the inability to retrieve the inhibitory meaning of the conditioned stimulus. He has found that when a salient feature of the extinction context is presented in a context other than the extinction environment, its presence reduces the probability of renewal. Similarly, Brooks (2000) has found that such retrieval cues can reduce the probability of spontaneous recovery. CERP incorporating retrieval cues might thus enhance generalisation of the extinguished drug responses to contexts other than the treatment setting. But what features might function as such retrieval cues? In animall studies these cues can be almost any kind of stimulus as long as it is perceived as a salient feature of the extinction context. It is not clear, though, what stimulus might function as such a retrieval cue in humans. Cunningham (1998) has suggested that perhaps wearing a specific clothing article during cue exposure sessions might endow this article with the function of a retrieval cue. Wearing this particular piece of clothing after discharge should help the patient to retrieve the cue exposure treatment setting and this should prevent renewal and spontaneous recovery of conditioned drug responding, thus limiting the probability of relapse. Another example of a potential retrieval cue is the use of a reminder card. Marlatt and Gordon (1985) describe the use of. a reminder card in their relapse prevention program. This card is used as a reminder of what to do in case of a lapse. In cue exposure treatment, such a card may be used as a reminder of the treatment setting. It may contain, for instance, a schematic representation of the rationale which reminds the client of the cue exposure treatment after discharge from a clinic, hence functioning as a retrieval cue.

\section{Conclusion}

Learning and motivation is a dynamic research area and thus contemporary learning theory continues to be an important resource for the implementation and development of behavioural therapy. However, this requires that both researchers as well as clinicians need to be aware of new developments within this area at all time. 
Based on principles derived from contemporary learning theory, CERP incorporating retrieval cues (e.g., a reminder card) can be a promising adjustment of standard cue exposure treatment. It does not require extending the length of treatment and may control for a renewal effect and spontaneous recovery at the same time. When cue reactivity is extinguished and recovery of conditioned drug responding can be prevented by cue exposure incorporating retrieval cues, it is predicted that relapse of addictive behaviour can be prevented more successfully. Whether CERP incorporating retrieval cues indeed more successfully prevents relapse of addictive behaviour is an important empirical question that needs to be addressed in future research. 


\title{
CHAPTER 5
}

\section{CONTEXTUAL CONTROL OF EXTINGUISHED CONDITIONED PERFORMANCE IN HUMANS*}

\begin{abstract}
Animal research has shown that extinguished conditioned performance is modulated by the environmental context in which extinction treatment has occurred. When the conditioned stimulus is presented outside the extinction context, conditioned responding is renewed. In two experiments, it was investigated whether a renewal effect can also be found in humans. In experiment 1 , a renewal effect was observed, although the effect was small and far from complete. In experiment 2, a more substantial renewal effect was observed. The extent to which this effect occurred depended on the degree to which context was manipulated. In a third experiment, the exact nature of the observed renewal effect was examined. Results indicated that, as opposed to animals, the extinction context does not modulate extinguished conditioned responding in humanss.
\end{abstract}

\footnotetext{
'This chapter is an adapted wersion of: Havermans, R.C. Keuker, J., Lataster, T, \& Jansen, A. (in press). Contextual control of extinguished conditioned performance in humans. Learning and Motivation.
} 
The renewal effect is one of the most important and relatively recent finding in the field of associative learning. Renewal refers to the effect in which conditioned performance is renewed after an extinction treatment. In 1979, Bouton and Bolles conducted three experiments investigating the contextual control of extinguished conditioned performance in rats, using conditioned suppression procedure. They found that when rats received extinction treatment in an envitonmental context different from the context in which these rats had previously received training, presenting the conditioned stimulus (CS) in the original training context at lest renewed conditioned suppression. It has been shown that this effect cannot be attributed to the extinction context acquiring a direct inhibitory association with the unconditioned stimulus (US) (see Bouton \& Peck, 1989). Rather, it seems that during extinction the CS becomes endowed with an inhibitory meaning modulated by the environmental context in which the extinction treatment has taken place. The CS has thus acquired an ambiguous meaning, as it both predicts the occurrence and the absence of the US. The meaning of the CS is disambiguated by contextual information. When the CS is presented within the extinction context, the inhibitory CS-no US association is retrieved. Context specificity of learned performance thus occurs in situations in which the CS has acquired such an ambiguous meaning (but see Hall \& Honey, 1990; Maes, Havermans \& Vossen, 2000). In a counterconditioning preparation, the CS also acquires an ambiguous meaning. The second llearned association then comes under contextual control, whereas the first learned meaning of the CS generalises across environmental contexts (see Bouton, 1994a). This implies that presentation of the CS outside the extinction context should lead to renewal of the conditioned response, whether or not the CS is presented in the original training environment. It has repeatedly been shown in animal research that the renewal effect is not merely restricted to the te-presentation of the $\mathrm{CS}$ in the original training context (see Bouton, 1993, 1994b). The renewal effect thus demonstrates that extinction is not the unlearning of an excitatory association as more traditional theories of associative learning predict (e.g., Rescorla \& Wagner, 1972; see also Rescorla, 2001).

The tenewal effect can be understood in terms of an interference effect, in which there is interference between different outcomes when predicted by a single CS. In other words, when wo distinct outcomes are predicted by one and the same cue and at some point in time one of these outcomes is more strongly predicted by the cue, this will interfere with responding to the other outcome. Similarly, interference between cues is observed when they predict one and the same outcome. An example of such cue interference concerns the blocking effect, in which learning that a cue predicts a specific outcome interferes with learning that a second cue predicts the same outcome (Kamin, 1969). Recent studies in humans reveal that a second leamed association can interfere with the original association when these associations share the same outcome (Matute \& Pineño, 1998). More recently, Pineño and Matute (2000) demonstrated in human subjects interference between outcomes when two sequentially leamed associations shared the same predicting 


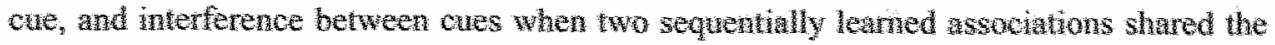

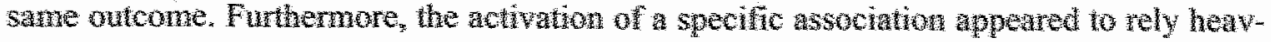

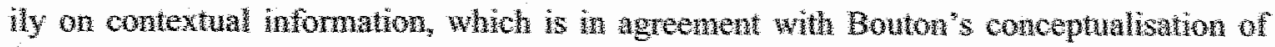

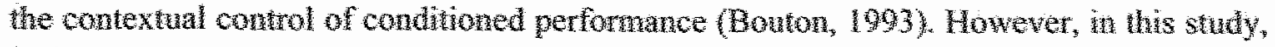

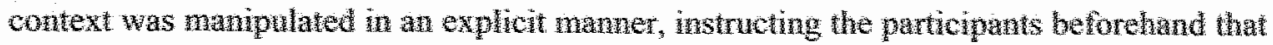
certan cues could predict diferent outcomes accorting to the contex m whoh these cues were prasented. Garcia-Gutierrez and fosas (2003) recenty also found some indication of a renewal effect in humans. In his sudy, participants had to predict the occurence of different symploms of gastric iness after he intake of diferent chemwally symhesised food items. Puticipants recerved temonstrations of hes of persons wang consumed diferent

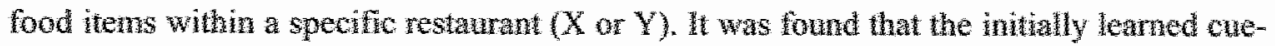
outcome associations were retreved acquistion context $X$ after an interterence procedure in context $\mathrm{Y}$. Althomg this effect certaniny resembles remewal of conditioned performanoe in aminals, context changes were estabished though descrintion rather than experience, as is the case in aximal studies on the contextual control of extinction.

Apparenty, renewal is a robast effect, which tends to occh in both animats and Wumans, although the retewal effect has not been studied that extensively in humans as of yet. In a firs expermen, the primary question of interest was whether a renewal eflect

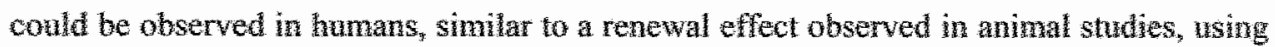
anore implicit condex manipulation than in the sudies of Pineno and Matute (2000), and Garcia-Gutierrez and Rosas (2003). A second experiment involved the investigation of factors affecting the potental contextral controw of extingushed condinoned performance in humans. One imporant factor is the degree in which a contex change is manipulated. Thomas, Larsen and Ayres (2003) demonstrated that renewed conditioned fear responding in rats using an $A B A$ renewal paradigm decreased as the $A$ and $B$ contexts became more similar. In a third experment, the exact nature of the renewal effect in humans was invesw tigated.

The questions of whether a renewal effect occurs in thumans and to what degree such an effect depends on context manipulation are relevant as the renewal effect holds great implications for the behavioural treatment of human psychopathology. One form of such a behavioural treatment is cue exposure therapy, which is based on the principle of extinction. It is assumed that certain cues elicit responses, which constitute, or lead to the expression of psychopathological behaviour and that repeated exposure to such cues will lead to the extinction of the abnormal behaviour. As Bouton and Swartzentruber (1991) have argued, animal research concerning the contextual control of conditioned responding shows that this particular assumption may be false. Gaining insight into what degree a renewal effect occurs in humans could thus be of great benefit to clinical research concerning cue exposure therapy. 


\section{Experiment 1}

In experiment 1 , it was investigated whether a renewal effect can also occur in human subjects, using an ABA renewal paradigm. Three different groups of participants received conditioning and a subsequent extinction treatment. One group received conditioning and extinction against the same background stimuli (context A). A second group received the extinction treatment against background stimuli (context B) different from the acquisition context $\mathrm{A}$. $\mathrm{A}$ third group received conditioning trials in context $\mathrm{A}$ and were subsequently exposed to context $\mathrm{B}$, but no extinction trials were presented in context $\mathrm{B}$. Contextual control of extinguished performance should be apparent in the participants having received the acquisition phase and the extinction treatment in different contexts.

\section{Method}

\section{Participants}

Forty-eight undergraduate students from Maastricht University participated in the present experiment ( 14 men and 34 women with a mean age of 20 years). Participants were randomly assigned to one of three different groups of equal size ( $n=16)$ : Group AAA, received acquisition and extinction in the same context; group ABA, received acquisition and extinction in different contexts; group NE, received conditioning trials in context $A$ but no extinction in context $B$. Participants received course credits upon completion of the experimental task.

\section{Procedure and Design}

A conditioned suppression task was employed to investigate whether renewal can occur in humans and was largely based on the task designed by Arcediano, Ortega and Matute (1996). In this task, participants played a videogame in which they had to prevent an impending invasion of hostile "Martians". This videogame was programmed in Experimental Run Time System (Berisoft, Germany) and run on an IBM-compatible personal computer. Participants completed the task indiwidually in a quiet research roon with the experimenter monitoring performance.

Premaining. Participants lirst received the verbal instruction from the experimenter that they would have to defend the planet Earth from the Martians by preventing the Martians from landing on the planet. They could do so by firing at the Martians with a laser gun (by pressing the spacebar of the keyboard). In the pretraining phase, Martians were presented every $600 \mathrm{~ms}_{\text {, }}$ one by one flying across the computer screen from the top center of the screen downward in $300 \mathrm{~ms}$. If one fired before the next Martian was displayed, an explosion instead of the Martian would be presented. Martians and explosions measured approximately 1.5 square $\mathrm{cm}$. In Figure 5.1 , the pictures representing the Martians and the explosion are displayed. The pretraining phase consisted of 100 
presentations of a Martian and lead to a constant, operant bar pressing response: Participants received the following additional instructions on screen (translated from Dutch):

"In this task, you need to prevent Martians from imading the planet. Approximately ewery half second, a Martian will try to land. In order fo destroy the Martians you will hawe to shoot them with your laser gun (by pressing the spacebar) BEFORE they can see you, that is just before a new Martian appears on screen. Do not fire too early, because you have only one shot per Martion. If you successfully destroy a Martian, an explosion instead of a Martian will appear on screen. At the end of this game you will attain feedback on the percentage of Martians you have desfroyed.

THE PLANET IS COUNTING ON YOU! DON'T LET THEM LANDI!"
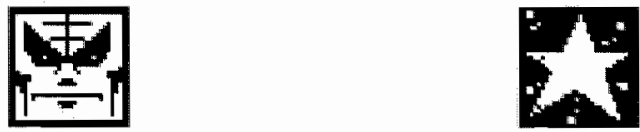

Figure 5.1. Picture of a Martian (left) and an explosion (right) as used in the present study.

Training. In the training phase, participants were told that the Martians have developed an antilaser-shield and that firing the laser gun while the shield is activated will reflect the shot disabling the gun for $4.8 \mathrm{~s}$. The Martians invade the planet at twice the speed while the laser gun is out of order. The antilaser-shield was displayed as a white intermittent flashing of the background screen colour (white/black. alternating every $100 \mathrm{~ms}$ ) with a duration of $600 \mathrm{~ms}$. First, the experimenter demonstrated in 4 trials what the antilaser-shield looked like and what would happen if one fires during presentation of the antilaser-shield. Next, the participant had to perform the task for himself, but now a $500 \mathrm{~Hz}$ computer generated tone immediately preceded the presentation of the antilaser-shield with a duration of $600 \mathrm{~ms}$. The tone thus functioned as a CS and the antilaser-shield as a US. All participants received the following instructions (translated from Dutch):

"The Martians have now developed an antilaser-shield. You have to keep firing your laser in order to preven them from landing. BUT BE CAREFUL, because when you fire when the shield is activated, your shot will be reflected, disabling your lase" gun. Immediarely thowands of Martians will land safely, whithout any interference. You can recognise the activation of the shield by a WHITE intermittent FLASHING of the background.

Remember! It only takes one shot when the antilaser-shield is acrivated, awd the Martians will invade successfully!!"

The training phase consisted of 20 conditioning trials with a variable intertrial interval (ITI), varying around a mean of $6 \mathrm{~s}$ (ranging from $4.8-7.2 \mathrm{~s}$ ). The first and every fourth trial functioned as assessment trials in which the tone lasted for $3 \mathrm{~s}$. These longer presentations of the CS allowed for a more sensitive measurement of conditioned suppression (see also Arcediano, Ortega \& Matute, 1996). Conditioned suppression was measured by calculating the suppression ratio, $\mathrm{X} / \mathrm{X}+\mathrm{Y}$, on the assessment trials with $\mathrm{X}$ being the number of bar presses during the $3 \mathrm{~S}-\mathrm{CS}$ and $\mathrm{Y}$ being the number of bar presses during the $3 \mathrm{~s}$ period directly preceding onset of the CS. Conditioning was considered successful when participants had a suppression ratio lower than 0.3 at the last training trial. 
One could argue that the term conditioned suppression does not apply to the present task. In animal research, conditioned suppression refers to a conditioned emotional response. In rats, the suppression response consists partly of freezing, a fear-motivated reaction. In the present task, the suppression response is not so much a conditioned emotional response because it is not fear driven. The Martian task might be described instead as an anticipated punishment procedure. Responses during the presentation of the white intermittent flashing of the background will be punished. In anticipating this punishment, participants eventually suppress their responses not only during presentation of the white flash, but also during presentation of the predictive tone stimulus. Therefore, the suppression of bar pressing that occurs while the predictive cue is present can be regarded as an anticipatory reaction to the passive avoidance of the white flash that is required by the game instructions. Thus, the term conditioned suppression, as used throughout this chapter, specifically refers to suppression ofl operant responding due to anticipated punishment and not to conditioned emotional responding.

Exfinction. Directly following training, participants from groups $\mathrm{AAA}$ and $\mathrm{ABA}$ received 20 extinction trials with the first and every fourth extinction trial as assessment trials and the same variable ITI as during the training phase. Extinction trials consisted of presentation of the CS alone. Participants from group NE received no extinction trials (i.e., no CSs and USs were presented), but they had to destroy the Martians for the same amount of time as the participants from the other two groups during extinction.

Extinction lest. At test, each participant received 8 extinction trials with the first and every fourth trial as assessment trials and the same variable ITI as during the training and extinction phase. The test phase immediately followed the extinction treatment.

Context manipulation. An important difference between the original task by Arcediano, Ortega and Matute (1996) and the task used in the present study is that the invading Martians do mot accumulate on screen during the game. This was done deliberately, because in the present study context was manipulated by changes in background screen colour. The accumulation of Martians on screen would have negatively affected context salience. Group AAA received the different phases of the task against one and the same background screen colour, blue or red counterbalanced. Group $\mathrm{ABA}$ received pretraining and training against a background $\mathrm{A}$ (blue or red counterbalanced), extinction against another background $B$ (red when $A$ had been blue, and vice versa), and the test against background $A$. Group NE received the same context manipulation as group ABA. The experimental design is displlayed in Table 5.1 .

Table 5.1

Design of experiment 1 .

\begin{tabular}{|c|c|c|c|c|c|}
\hline & & \multicolumn{4}{|c|}{ Phase } \\
\hline & & Pretraining & Training & Extinction & Test \\
\hline \multirow{3}{*}{ Group } & AAA & A & $\mathrm{A}[\mathrm{CS}-\mathrm{US}]$ & $\mathrm{A}[\mathrm{CS}]$ & $\mathrm{A}[\overline{\mathrm{CS}}]$ \\
\hline & $A B A$ & A & A[CS - US] & $\mathrm{B} \llbracket \mathrm{CS} \rrbracket$ & $\mathrm{A}[\mathrm{CS}]$ \\
\hline & $N E$ & A & A[CS - US] & $\mathrm{B}$ & $\mathrm{A}[\mathrm{CS}]$ \\
\hline
\end{tabular}

Note. $A$ and $B$ represent the background screen collour (blue versus red counterbalanced) of the "Martians" task. 


\section{Results and Discussion}

For all reported analyses, a level of significance of $p<.05$ was used, unless stated otherwise. In Figure 5.2, the mean suppression ratios per group and per assessment trial; as measured throughout the experiment, are displayed.

Conditioning. Conditioning would be regarded successful when a suppression ratio lower than 0.3 was observed on the last training trial. Although most participants met this criterion, 12 participants failed to do so; 4 participants from group AAA, 6 participants from group $\mathrm{ABA}$ and 2 participants from group $\mathrm{NE}$. The data from these participants were thus excluded from the following analyses.

To test whether the training phase led to a gradual increase in the strength of conditioned suppression, a two-way analysis of variance (ANOVA) was conducted with Trial ( 1 , $4,8,12,16$, or 20 ) as a within-subject factor, Group (AAA, ABA, or NE) as a betweensubjects factor, and suppression ratio calculated over the 6 assessment trials during the training phase as the dependent variable. A main effect for Trial was found, $F(5,100)=$ 7.91. No other effects were found, $F_{S}<1$. These results indicate that training led to a progressive increase in the strength of conditioned suppression across groups.

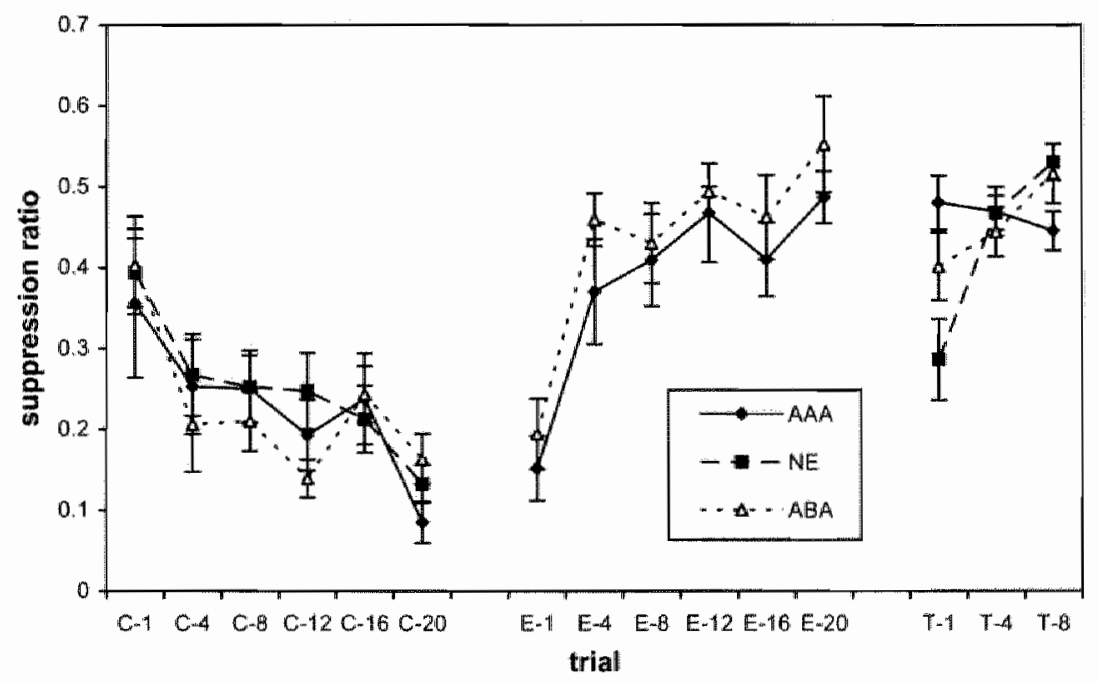

Figure 5.2. Mean suppression ratio per assessment trial ( \pm SEM) for each of the three groups. C stands for conditioning $\mathrm{E}$ stands for extinction and $\mathrm{T}$ stands for test.

Extinction. To test whether the extinction treatment led to the gradual extinction of conditioned suppression, a two-way ANOVA was run with Trial $(1,4,8,12,16$, or 20$)$ as 
a within-subject factor, Group (AAA versus $A B A$ ) as a between-subjects factor, and suppression ratio callculated over the 6 extinction assessment trials as the dependent variable. A main effect of Trial was tound, $F(5,100)=13.69$. No Trial $x$ Group interaction effect was found, $F(5,100)=0.15$. No effect of Group was found, $F(1,20)=1.98$. These results indicate that the extinction treatment led to the extinction of conditioned suppression in both groups.

Renewal. To test for a potential renewal effect, a two-way ANOVA was conducted with Trial (the last extinction trial versus the first test trial) as a within-subject factor, Group (AAA versus ABA) as a between-subjects factor, and suppression ratio calculated over the last extinction assessment trial and the first test assessment trial as the dependent wariable. A trend towards an effect for Trial was found, $F(1,20)=3.75, p<.10$. A trend towards a Trial $x$ Group interaction effect was found, $F(1,20)=3.14, p<.10$. No effect of Group was found, $F<1$.

Planned comparisons, analysing conditioned suppression on the first test assessment trial, revealed a significant difference in suppression ratio for the first test trial between group AAA and group NE, $F(1,25)=9.75$. No difference in suppression ratio on the first test trial between group $A A A$ and $A B A$ was found, $F(1,21)=2.25$, and no difference was found in suppression ratio on the first test trial between groups $A B A$ and $N E$, $F(1,23)=2.77$.

The present results of interest indicate that the participants in group $A B A$ showed no direct evidence for renewal of conditioned suppression. The results however display a clear trend towards a renewal effect. Furthermore, if no renewal had occurred at all, one would expect that the suppression ratio on the first test trial for the participants in group ABA would have differed significantly from conditioned performance on the first test trial for the participants in group NE. The fact that this was not the case indicates that some renewal of conditioned suppression may have taken place, although this renewal effect is admittedly small.

Although the experimenter monitored performance of the participants throughout the experiment, no feedback was provided about their performance by the experimenter. This may have led to insufficient learning of the operant response and the subsequent finding that $25 \%$ of all the participants failed to meet the conditioning criterion. This loss of data may have negatively affected the power of the statistical analyses and caused a type II error. An alternative explanation for the absence of a substantial renewal effect in this experiment might be that the context manipulation was not strong enough; that is, the participants did not perceive the changes in background screen colour as a fundamental change from one context to another. It is important to note that the Martians displayed in the task were the stme colour throughout the entire experiment (i.e., white), and thus formed a common element between the different experimental contexts. It is passible that this common element reduced the discrepancy between contexts $A$ and $B$, hence promoting gener- 
alisation of extinguished behaviour. This notion then leads to the prediction that a more complete context manipulation should lead to a more substantial renewal effect.

\section{Experiment 2}

In experiment 1 , no substantial renewal was observed. It is suggested that this may be due to an incomplete context manipulation. Indeed, animal research has shown that renewal of extinguished conditioned performance depends on the degree to which the test context perceptually differs from the extinction context (Thomas, Larsen, \& Ayres, 2003). Therefore, a second experiment was conducted in an attempt to replicate the results from experiment 1 and to investigate whether the degree to which a context switch is established determines the extent to which a potential renewal effect can be observed.

\section{Method}

\section{Participants}

A total of 48 undergraduate students from Maastricht University were recruited for participation in the present experiment ( 10 men and 38 women with a mean age of 21 years). Participants were randomly assigned to 4 different groups (AAA, ABA, ABAt, or NE) of equal size $(n=12)$. Participants received course credits upon completion of the task.

\section{Procedure and Design}

The design for experiment 2 is displayed in Table 5.2. The same procedure was used as described in experiment 1. Context was again manipulated by changes in background screen colour; blue wersus yellow. However, in group ABAt, switches in background screen colour were additionally accompanied by changes in the colour of the invading Martians (white when the background colour is blue, and red when the background colour is yellow). In group ABA, the colour of the Martians (white or red) displayed during extinction would remain the same during test, despite a change in background colour.

In experiment 1, participants indicated after the experiment that they found the task to be rather difficult. Indeed, the participants who failed to meet the conditioning criterion showed highly irregular responding (i.e., shooting Martians) throughout the entire experiment. Feedback may improve performance dluring the pretraining phase and hence lead to better performance in the following phases of the task. Therefore, in the present experiment, the experimenter monitored performance during the pretraining phase and gave concurrent verbal feedback upon performance during this phase. The experimenter did not give any feedback during the rest of the experiment. 
Dasign of experiment 2 .

\begin{tabular}{|c|c|c|c|c|c|}
\hline & \multicolumn{4}{|c|}{ Phase } \\
\hline & & Pretraining & Training & Extinction & Test \\
\hline \multirow{4}{*}{ Group } & $\overline{A A A}$ & $\mathrm{Aa}$ & $\mathrm{Aa}[\mathrm{CS}$ - US] & $\mathrm{Aa}[\mathrm{CS}]$ & AalCS] \\
\hline & $A B A+$ & Aa & Aa[CS - US] & $\mathrm{Bb}[\mathrm{CS}]$ & $\mathrm{Aa}[\mathrm{CS}]$ \\
\hline & $A B A$ & $\mathrm{Aa}$ & AarCS - USI & $\mathrm{Bb}[\mathrm{CS}]$ & Ablcs] \\
\hline & NE & $\mathrm{Aa}$ & AalCS-US] & $\mathrm{Bb}$ & Aa[cs] \\
\hline
\end{tabular}

Note. A and $\mathrm{B}$ represent the background screen colour (blue versus yellow, counterbalanced) of the "Martians" task "whereas a and b represent the colour of the Martians (or explosions) (white versus red, couriterbalanced).

\section{Results and Discussion}

The results in Figure 5.3 display the mean suppression ratio per assessment trial, as measured throughout the experiment, and per group.

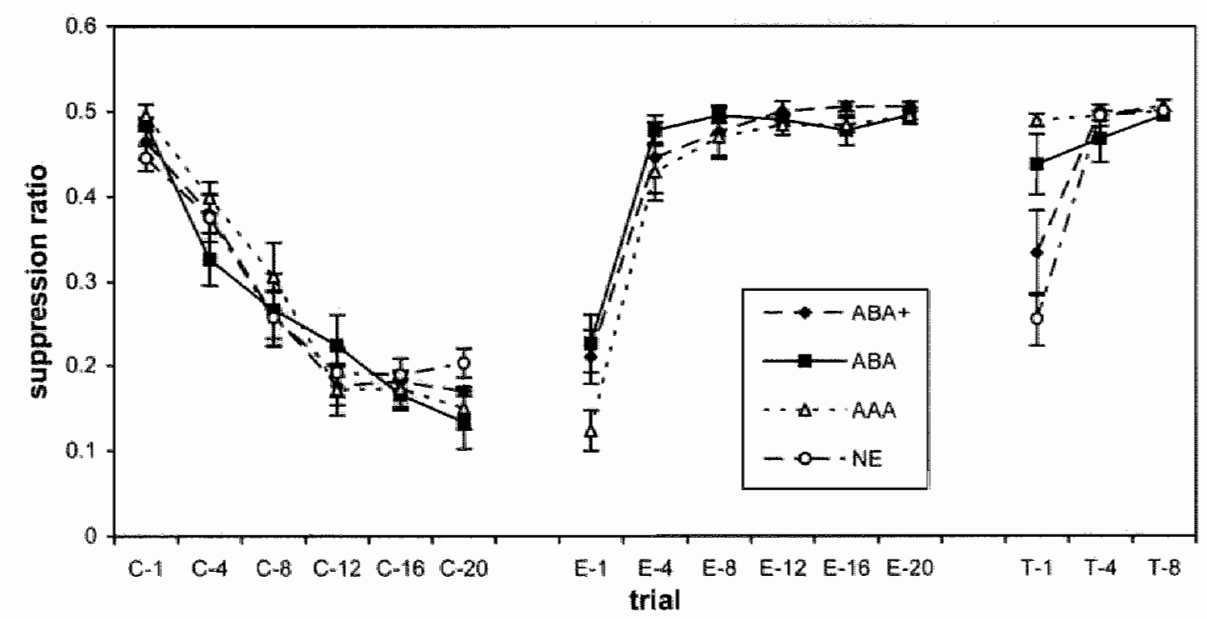

Figure 5.3. Mean suppression ratio per assessment trial for each of the four different groups, minus the participants who failed to meet the conditioning criterion. $C$ stands for conditioning, $E$ stands for extinction and $T$ stands for test. Error bars represent standard errors of the means.

Conditioning. Conditioning would again be regarded as successful when a suppression ratio lower than 0.3 was observed on the last training trial. A total of 3 participants 
failed to meet this criterion; I participant from group AA, 1 participant from group $\mathrm{ABA}+$ and 1 participant from group NE. The data from these participants were thus excluded from the following analyses.

To test whether the training phase rendered a gradual increase in conditioned suppression, a two-way ANOVA was conducted with Trial $(1,4,8,12,16$, or 20$)$ as a withinsubject factor, Group (AAA, ABA, ABAt, or NE) as a between-subjects factor, and suppression ratio calculated over the 6 assessment trials during the training phase as the dependent variable. A main effect for Trial was found, $F(5,220)=127.58$. No Trial $x$ Group interaction effect was found, $F(15,220)=1.30$, and no effect of Group was found, $F<1$. These results indicate that training led to a progressive increase in the strength of conditioned suppression across groups.

Extinction. To test whether the extinction treatment led to the successful extinction of conditioned suppression, a two-way ANOVA was run with Trial $(1,4,8,12,16$, or 20$)$ as a within-subject factor, Group (AAA, $A B A$, or $A B A+$ ) as a between-subjects factor, and the suppression ratio calculated over the 6 extinction assessment trials as the dependent variable. A main effect of Trial was found, $F(5,155)=119.64$. No Trial x Group interaction effect was found, $F(10,155)=1.39$, and no effect of Group was found, $F(2,31)=$ 1.77. These results indicate that the extinction treatment led to the successful extinction of conditioned suppression.

Renewal. To test for a potential renewal effect, a two-way ANOVA was conducted with Trial (the last extinction trial versus the first test trial) as a within-subject factor, Group (AAA, $\mathrm{ABA}$, or $\mathrm{ABA}+$ ) as a between-subjects factor, and suppression ratio calculated over the last extinction assessment trial and the first test assessment trial as the dependent variable. An effect of Trial was found, $F(1,31)=13.34$, a Trial x Group interaction was found, $F(2,31)=5.16$, and an effect of Group was found, $F(2,31)=4.17$. These results clearly imply that a renewal effect has occurred. The significant Trial $x$ Group interaction also indicates that this renewal effect may have specifically occurred in one particular group. Post hoc $t$ tests, testing each group separately for a potential renewal effect by comparing conditioned suppression on the last extinction trial with conclitioned sup pression on the first test trial per group revealed that renewal was evident in group $\mathrm{ABA}+$, $t(10)=3.32$. No direct evidence for a renewal effect was found for group $A B A, t(11)=$ 1.57 , and no renewal was found for group $\mathrm{AAA}, t(10)=0.56$.

Planned comparisons, analysing conditioned suppression on the first test assessment trial, revealed a significant difference in suppression ratio on the first test trial between group $\mathrm{ABA}+$ and $\mathrm{AAA}, F(1,21)=9.55$. A difference was found between $\mathrm{ABA}$ and $\mathrm{NE}, F(1,22)=14.53$. A significant difference was found between group AAA and NE, $F(1,21)=51.65$. No difference was found in suppression ratio on the first test trial between groups $A B A+$ and $N E, F(1,21)=1.79$. No difference was found in suppression ratio between group AAA and ABA, $F(1,22)=1.86$. No significant difference in suppres- 
sion ratio for the frist test trial between group $\mathrm{ABA}+$ and group $\mathrm{ABA}$ was found, $F(1,22)$ $=2.96$.

The results of interest indicate that, as in experiment 1 , group ABA shows a minor trend towards a renewal effect. However, renewal was far more substantial for group $\mathrm{ABA}+$ that received a more complete context manipulation than group $\mathrm{ABA}$. Conditioned suppression at test for the participants in group $A B A+$ did not differ from the degree of conditioned suppression by the participants from group NE who had received no extinction treatment: It is thus shown that renewal can be observed in humans and that in accordance with the animal literature this depends on the extent to which the test context differs from the extinction context.

Although a strong renewal effect was observed in this experiment, it remains unclear whether the observed return of conditioned responding after extinction is due to the extinction context modulating the activation of the inhibitory meaning of the CS. The present renewal effect can be explained by arguing that a context switch will lead to generalisation decrement and thus to a loss of inhibited or excitatory responding. To test whether the initial context switch from acquisition context to the extinction context had led to a loss of conditioned suppression for participants in groups $\mathrm{ABA}$ and $\mathrm{ABA}+$ as opposed to the participants in group AAA, a two-way ANOVA was conducted with Trial (the last conditioning assessment trial versus the first extinction trial) as a within-subject factor, Group ( $\mathrm{ABA}, \mathrm{ABA}+$, or AAA) as a between-subjects factor, and suppression ratio as the dependent variable. No main effect for Trial was found, $F(1,31)=3.66$, and no effect of Group was found, $F(2,31)=1.60$. However, a significant Trial $x$ Group interaction effect was found, $F(2,31)=3.50$, indicating that there may have been some generalisation decrement but limited to a specific group. To test this suggestion, post hoc $t$ tests were conducted comparing conditioned suppression on the last conditioning trial and the first extinction trial within each group (i.e., groups ABA, ABAt and AAA). No difference in conditioned suppression was found for group $\mathrm{ABA},(10)=1.27$, but a significant difference in suppression was found for the participants in group $A B A+, t(11)=3.13$. As expected, no difference in conditioned suppression was found for group AAA, $t(10)<1$. These results imply that some generalisation decrement occurred and that the strong renewal effect observed for the participants in group $\mathrm{ABA}+$ can at least partially be ascribed to the formation of a context A-CS configural cue being associated with the US during the acquisition phase. 


\section{Experiment 3}

In experiments 1 and 2, evidence for renewed conditioned responding after an extinction treatment in humans was found. Although this suggests that as in animals, the CS acquires an ambiguous meaning that is modulated by the environmental context, the observed renewal effect can also be explained by stating that the CS and the acquisition context taken together form a configural stimulus that is associated with the US (Pearce, 1987). This should lead to renewed conditioned responding after an extinction treatment in context $B$ when the $\mathrm{CS}$ is presented in the original conditioning context A. Another alternative explanation is that during the extinction phase, the extinction context $B$ becomes an inhibitory stimulus. In this case no extinction of conditioned responding to the CS will have taken place allowing for the return of conditioned responding when the CS is presented outside the extinction context. Therefore, a third experiment was conducted to replicate and further examine the exact nature of the present observed renewal effect in humans.

\section{Method}

\section{Participants}

A total of 54 undergraduate students from Maastricht University were recruited for participation in the present experiment ( 16 men and 38 women with a mean age of 20 years). Participants were randomly assigned to 3 different groups $(\mathrm{ABA}, \mathrm{ABB}$, or $\mathrm{ABC}$ ) of equal size $(n=18)$. Participants received course credits upon completion of the task.

Table 5.3

Design of experiment 3.

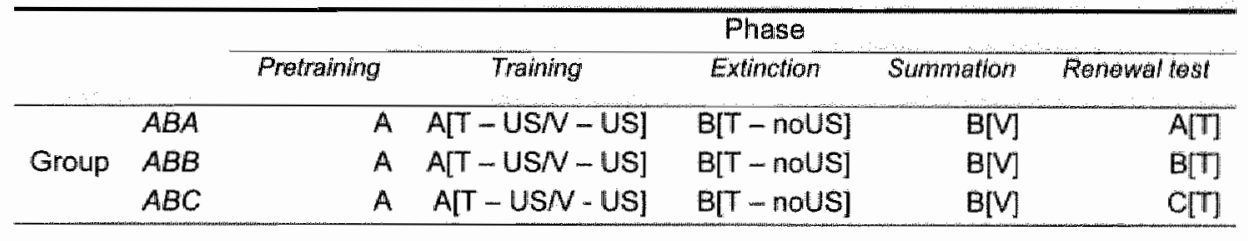

Note. A, B and C represent the different experimental contexts of the "Martians" task (blue background with orange Martians, red background with white Martians, or a yellow background with purple Martians counterbalanced). T and $V$ represent the auditory and visual CS respectively.

\section{Procedure and Design}

The design for experiment 3 is displayed in Table 5.3. The same procedure was used as described in experiments $I$ and 2. Context was manipulated by changes in background screen colour and the colour of the Martians; a blue background with orange Martians, a yellow background with 
purple Martians, or a red background with white Martians. During the acquisition phase, two distinct CSs were paired with the US. The CSs comprised an auditory stimulus $T$ as described in the previous experiments and a visual stimulus $V$. This latter stimulus consisted of the 600 ms presentation of 3 vertically spaced cyan coloured squares of approximately 3 square $\mathrm{cm}$ at the centre of the screen. The acquisition phase consisted of 40 trials; 20 acquisition trials for each CS. As in the previous experiments, the first and every fourth trial of each CS were assessment trials in which the CS was presented for 3 s. The order of trials during this acquisition phase was determined randomly with the limitation of no more than 3 consecutive trials of the same type, and was equal for all participants.

All participants received an initial context $\$$ witcla, from the acquisition phase in context $A$ to extinction in context $\mathrm{B}$. If conditioned responding is context specific, this context switch should lead to a loss of responding due to generalisation decrement. The extinction phase consisted of 12 trials of nonreinforced exposure to $T$ with the first and every fourth trial functioning as assessment trials. After the extinction of responding to $T$, a summation test followed which comprised a single nonreinforced 3-s presentation of $V$ in context $B$. If the extinction context $B$ has acquired an inhibitory association with the US, one would expect attenuated conditioned responding to $V$ on the summation test.

The summation test was followed by a single 3-5, monreinforced presentation of $T$ in either the original acquisition context $A$ (group $A B A$ ), the extinction context $B$ (group $A B B$ ), or in a novel context $\mathrm{C}$ (group $\mathrm{ABC}$ ), comprising the renewall test. If renewal is due to the $\mathrm{CS}$ acquiring an ambiguous meaning controlled by the extinction context, then one would expect to find renewal in both group $\mathrm{ABA}$ and $\mathrm{ABC}$, as opposed to group $\mathrm{ABB}$.

\section{Results and Discussion}

Figure 5.4 displays the mean conditioned suppression to $\mathrm{T}$ and $\mathrm{V}$ during conditioning, the extinction phase and the summation test for all participants, regardless of group assignment.

Condinioning. Agaim, a conditioning criterion was used. Conditioning was considered to be successful when a suppression ratio lower than 0.3 was measured for each CS (T and $V$ ) on the last training assessment trials. A total of 16 participants failed to meet this criterion: 5 participants in group $A B A, 5$ in group $A B B$, and 6 in group $A B C$. The data of these participants were excluded from further analyses.

On the data of the remaining 38 participants, a three-way ANOVA was conducted, with CS (T versus V) and Trial (conditioning assessment trials 1 to 6 ) as the within-subject tactors, Group ( $\mathrm{ABA}, \mathrm{ABB}$, or $\mathrm{ABC}$ ) as a between-subjects factor, and suppression ratio as calculated for the 6 conditioning assessment trials as the dependent variable. A main effect of $\mathrm{CS}$ was found, $F(1,35)=8.67$, indicating stronger conditioned suppression to CS V than to CS T. An effect of Trial was found, $F(5,175)=31.59$, reflecting an increase in the strength of conditioned suppression with progressive conditioning. No effect of Group was 
found, $F(2,35)=2.25$. A CS $\times$ Trial interaction was found, $F(5,175)=5.56$, indicating a differential increase in strength of conditioned suppression comparing suppression to $\mathrm{CS} \mathrm{V}$ and $\mathrm{T}$. This effect can be ascribed to the fact that for each participant the first conditioning trial consisted of the reinforced presentation of $T$. Therefore, the first presentation of $V$ may have lead the participants to behave more cautiously than normal (expressed as suppression of the operant response) due to their prior experience with T. No other effects were found, $F \mathrm{~s}<1$.

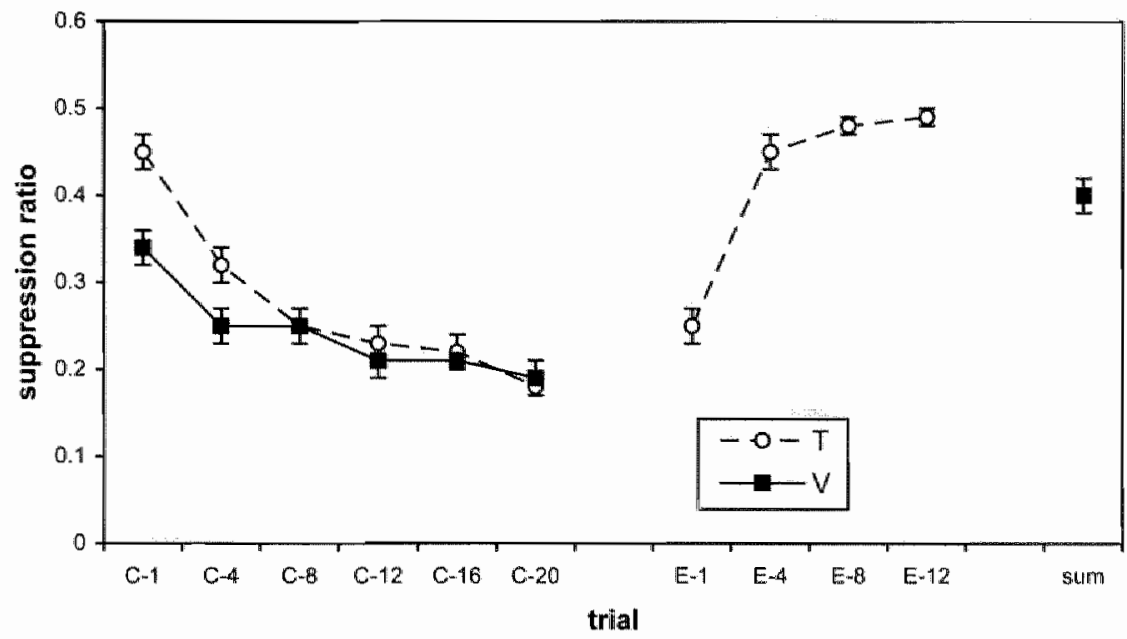

Figure 5.4. Mean suppression ratio per assessment trial of the conditioning phase, the extinction phase and the summation test for all participants regardless of group assignment (minus the participants who failed to meet the conditioning criterion). $C$ stands for conditioning. $E$ for extinction and 'sum' refers to the summation test. $T$ and $V$ refer to the auditory and visual CS respectively. Error bars represent standard errors of the means.

Extinction. To test whether extinction of conditioned suppression to the tone Thad been successful, a two-way ANOVA was conducted with Trial (extinction assesstment trials 1 to 4 ) as a within-subject factor, Group (ABA, ABB, or ABC) as a between-subjects factor, and conditioned suppression to the tone on the extinction assessment trials as the dependent variable. A main effect of Trial was found, $F(3,105)=64.53$, reflecting the rapid extinction of conditioned suppression to the tone $T$. No effect of Group was found, $F$ $<1$, and no interaction was found, $F(6,105)=2.04$, indicating that the pattern of extinction was uniform across groups.

Generalisation decrement. In the previous experiments, some generalisation decrement was found, referring to the loss of conditioned responding due to a context switch after the acquisition phase. To test whether generalisation decrement had occurred in the present experiment, a two-way ANOVA was conducted with conditioned suppression to T' 
on the last conditioning assessment trial and the first extinction trial as a within-subject factor and Oroup $(\mathrm{ABA}, \mathrm{ABB}$, or $\mathrm{ABC})$ as a between-subjects factor. $\mathrm{A}$ main effect for Trial was found, $F(1,35)=19.59$, implying a loss of strength of conditioned suppression to $\mathrm{T}$ with the context switch from $\mathrm{A}$ to $\mathrm{B}$ due to generalisation decrement. No effect of Group was found, $F(2,35)=1.57$, and no interaction was found $F(2,35)=2.93$.

Summation rest. To test whether the extinction of conditioned suppression to $\mathrm{T}$ was the result of an inhibitory context B-noUS association, a summation test was conducted following the extinction phase. In context B, a single nonreinforced presentation of $V$ comprised the summation test. Conducting a two-way ANOVA, conditioned suppression to $\mathrm{V}$ on the summation test was compared with conditioned suppression to $\mathrm{T}$ on the last extinction trial. A significant difference in conditioned suppression to $T$ and $V$ was found, $F(1,35)=17.46$, indicating that the extinction context did not completely inhibit conditioned suppression to $\mathrm{V}$. No other effects were found, $F_{\mathrm{S}}<1$. An additional t-test was conducted comparing suppression to $\mathrm{V}$ on the summation test with responding to $\mathrm{V}$ at the end of acquisition. A significant difference in conditioned suppression was found, $t(37)=$ 10.33, indicating a loss in conditioned suppression to $\mathrm{V}$ at the summation test. Although apparently conditioned suppression to $\mathrm{V}$ was attenuated when presented in context $\mathrm{B}$, participants still showed conditioned suppression to $\mathrm{V}$ within this context, as opposed to $\mathrm{T}$. This then implies that $\mathrm{V}$ was still a potent $\mathrm{CS}$ and that context $\mathrm{B}$ alone was not controlling. extinction.

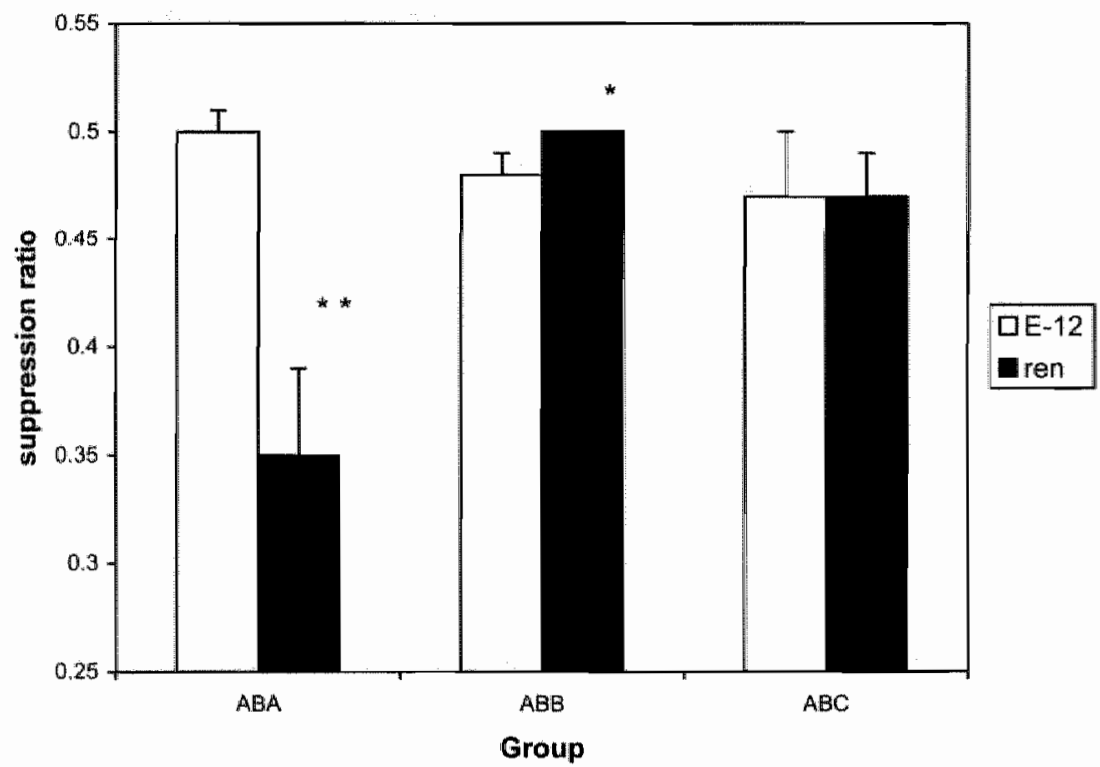

Figure 5.5. Mean suppression ratio for $T$ at the last extinction trial (E-12) and the renewal test ("ren") for each separate group plus standard errors of the means. ${ }^{*}=p<.05{ }^{* *}=p<.01$. 
Renewal test. Figure 5.5 displays the mean conditioned suppression to T at the last extinction trial versus the renewal test for each separate group. To test whether renewal had occurred a two-way ANOVA was conducted with Trial (the last extinction trial versus the renewal test trial) as a within-subject factor, Group $(\mathrm{ABA}, \mathrm{ABB}$, or $\mathrm{ABC}$ ) as a between-subjects factor, and conditioned suppression to $T$ on the last extinction trial and the renewal test as the dependent variable. A main effect of Trial was found, $F(1,35)=7.52$, indicating differential conditioned responding comparing the last extinction trial with the renewal test. No effect of Group was found, $F(2,35)=3.18$, but the Trial $x$ Group interaction proved to be significant, $F(2,35)=11.60$, implying that the effect of Trial was probably restricted to a particular group (or groups). These results are indicative of a renewal effect, as conditioned suppression appears to be stronger on the renewal test than on the last extinction trial.

Post hoc $t$-tests were conducted to examine which of the three groups showed a renewal effect. As expected, a renewal effect was apparent for participants in group ABA, $t(12)=3.46$. A significant difference in conditioned suppression was also found for the participants in group $A B B, t(11)=2.35$. As can be inferred from Figure 5.5, however, this difference is due to the fact that participants in this group showed less conditioned suppression to $T$ at the renewal test than at the last extinction trial and thus can be ascribed to progressive extinction of conditioned suppression to $T$. Somewhat unexpectedly, no indication of a renewal effect was found for group $\mathrm{ABC}, t(12)<1$.

In addition, planned comparisons were conducted, comparing the groups on conditioned suppression to $T$ on the renewal test. Participants in group ABA demonstrated more conditioned suppression on the renewal test than the participants in group $\mathrm{ABB}, F(1,24)=$ 11.33, reflecting the return of conditioned suppression to T for group ABA. Participants in group $\mathrm{ABA}$ also showed more conditioned suppression on the renewal test when compared with group $\mathrm{ABC}, F(1,25)=7.08$. No difference in conditioned suppression to $\mathrm{T}$ on the renewal test was found between groups $\mathrm{ABB}$ and $\mathrm{ABC}, F(1,24)=2.10$.

The results indicate the renewal of extinguished performance, as in experiments 1 and 2, but this apparent renewal effect was restricted to the acquisition context $A$ (ABA paradigm). The context specific renewal effect can be explained by arguing that conditioned suppression to $T$ was context specific to begin with. Such context specificity can be accounted for by presuming that the conditioning trials in context $A$ led to the formation of a configural context A-CS stimulus being associated with the US. According to this expla. nation, the initial context switch should have led to generalisation decrement, leading to a loss of conditioned suppression. Indeed, such generalisation decrement appears to have occurred. Renewal of the CR should thus be expected only to occur when the CS is presented in the original acquisition context $A$, but not in a novel context $C$. 


\section{General Discussion}

In the present study, the question whether renewal of conditioned performance can be demonstrated in humans was investigated using a behavioural conditioned suppression preparation. The results from the three experiments indicate that renewall can indeed be observed in humans. As in animal research, the degree of renewal depends on the degree of the context switch, the larger the context change the larger the renewal, as was demonstrated in experiment 2 (see also Thomas, Larsen, \& Ayres, 2003). However, in contrast with animal research, the results of experiment 3 indicate that such renewal was restricted. to the ABA renewal paradigm. When the CS T was presented at test in a novel context $C$, no renewal of extinguished conditioned suppression occurred. One could argue that the absence of a clear $A B C$ renewal effect results from the extinction context $B$ acquiring a direct inhibitory association with the US during extinction treatment. However, responding to both CS T and CS V in extinction context B should then have been attenuated to an equal extent. This was not the case. After complete extinction of conditioned suppression to $T, V$ still elicited suppression in context $B$. The present pattern of results (i.e., ABA renewal, but no $A B C$ renewal) then can be explained by arguing that during conditioning configural context A-CS stimuli were formed and associated. with the occurrence of the US. The loss of conditioned suppression to $\mathrm{T}$ on the first extinction trial and to $\mathrm{V}$ at the summation test then can be explained as generalisation decrement from the acquisition stimuli.

An alternative explanation for the present results is that the switch from acquisition context A to extinction context B led participants to adopt the rule that suppression to $T$ is only necessary in context A. The adoption of such a rule would lead participants to demonstrate renewal of conditioned suppression when tested in A, but not when tested in any other context. This notion then implies a comparative difference for the basis of a renewal effect in humans and animals. In other words, in animals renewal is controlled by a basic associative learning system, whereas in humans effortful capacity-limited cognitive processing may override the associative control of extinction. The question that arises is how to test such a hypothesis. Assuming that rule learning requires effortful cognitive processing one could attempt to replicate the present experiments adding a group of participants having to perform the same conditioned suppression task, but in addition is instructed to simultaneously perform a cognitive distraction task. This cognitive task should interfere with rule learning allowing for more associative control of conditioning suppression of responding and the subsequent extinction of such conditioned suppression. Whether such a procedure does lead to the demonstration of $\mathrm{ABC}$ renewal, however, requires further research. 


\section{Implications for behaviour therapy}

Although the present study found no evidence of the extinction context modulating extinguished behaviour, the successful demonstration of the return of conditioned suppression with the ABA paradigm is nonetheless of particular importance for clinical research investigating the efficacy of cue exposure therapy in the treatment of abnormal behaviour such as anxiety disorders, eating disotders and addictive behaviours (see Jansen, 1998). Learning theory constitutes the rationale of this form of treatment in which it is proposed that certain cues function as CSs elliciting cue reactivity conducive to the expression of abnormal behaviour. For instance, when one considers alcoholism, it is argued that the perceptual characteristics of one's favourite drink become associated with the intake of alcohol (i.e., the effect of alcohol). These alcohol cues thus come to elicit conditioned alcohol responses, or alcohol cue reactivity, which consists of an increase in the subjective urge to drink alcohol and psychophysiological responses (e.g., changes in heart rate and skin conductance level). Such alcohol cue reactivity has been found to increase the likelihood of excessive drinking behaviour (see Drummond, 2000). In cue exposure therapy, the alcohollic patient will be exposed to his fawourite alcoholic drink, but he is prevented from actual drinking. At first, this will elicit a near irresistible urge to drink, but, with repeated exposure, this urge extinguishes. However, the present results cleanly show that such extinction may be insufficient. Since not only a patient's favourite alcoholic drink, but also the environmental context in which the patient used to drink may serve as an alcohol CS, the extinguished cue-elicited urge to drink can reappear when the patient is exposed to his favourite alcoholic drink within the alcohol drinking context. Indeed, Conklin and Tiffany (2002) showed that although cue exposure therapy can lead to the successful extinction of drug cue reactivity, it does not prevent relapse of addictive behaviour. This inefficacy of cue exposure therapy can be explained by a potential renewal effect. Corroborative evidence for such a view comes from a recent study by Collins and Brandon (2002) who found in social drinkers that subjective and psychophysiological alcohol cue reactivity can be renewed after cue exposure therapy when the alcohol cues are presented in alting different from the treatment enviromment. These studies and the present results argute that extinction-based behavioural treatments of addiction (i.e., cue exposure therapy) will only prove to be beneficial when conducted within the environment in which the patient used to take drugs or alcohol (see also Havernans \& Jansen, 2003). 
ExcESSIVE APMETTE

82 


\title{
CHAPTER 6
}

\section{CONTEXT DEPENDENT ACCESS TO ALCOHOL-RELATED CONCEPTS STORED IN MEMORY*}

\begin{abstract}
In the present study, it was investigated whether a verbal alcohol-related prime would pronote accessibility of alcohol-related concepts stored in memory, and if so, whether such a priming effect would be more pronounced in a drinking-related environment. Participants were instructed to generate sentences with alcohol-ambiguous words after the presentation of a verbal alcohol-related stimulus, and after the presentation of a meutral prime in either a pub-like environment, or in an office-like setting. Participants generated more alcohol-related sentences in the pub than in the office. Presentation of the alcohol prime promoted the accessibility of alcahol-related concepts onlly in the participants having completed the sentence generation task in the office environment. The restilts suggest that rather than facilitating the priming of atcohol cognitions, a drinking-related context functions as an alcohol cue directly promoting the success to alcohol-related concepts stored in memory.
\end{abstract}

"This chapter is an adapted version of: Havermans, R.C., Bylois, E., Vancleef, L., Wiers, R.W., Jansen, A. (in press). Context dependent access to alcohol-related concepts stored in memory. Alcohol Research. 
Learning and memory play an important role in the dewelopment and maintenance of addictive behaviour. In general, it is argued that drinking experience leads to the storage of alcohol-related information in associative memory networks containing a central conceptwal node "alcohol" and surrounding nodes describing cues and effects related to alcohol use (Wiers, et al., 2002). Due to frequent alcohol use, this alcohol-associative memory network will become increasingly elaborate and more tensely interconnected, and hence more accessible. Access to such allcohol-related concepts from memory are thought to be conducive to the expression of alcohol drinking behaviour (Earlywine, 1995).

Priming can facilitate access to these alcohol-related representations. In many studies, word association tasks have been used to study the activation of alcohol-related cognitions. For instance, Stacy, Leigh and Weingardt (1994) primed alcohol-related cognitions by letting participants read alcohol outcome expectancies and asking the participants to write down the first behaviour that pops in mind. This study revealed that, in line with the assumption that alcohol-related cognitions are stored in memory in an associative network, hervier drinkers reported more alcohol-related behaviours. Stacy, Leigh and Weingardt (1997) found similar results when they primed participants using alcohol-ambiguous words. Memory activation of alcohol cognitions has indeed been found to predict drinking behaviour. For instance, Roehrich and Goldman (1995) found that priming of alcohol outcome expectancies led to an increase in alcohol drinking behaviour. In addition, Stacy (1997) has reported results from a large prospective study showing that memory activation of alcohol-related cognitions predicts drinking behaviour.

The observed priming effect of alcohol-related representations shows considerable conceptual overlap with an associative learning oriented view on alcohol use, which states that alcohol drinking behaviour leads to the formation of alcohol-related associations between alcohol cues, such as the perceptual characteristics of one's favourite alcoholic drink, and an alcohol effect. Alcohol cues come to predict an alcohol effect and thus come to elicit che reactivity, such as an increase in the subjective urge to drink and psychophysiological responses (e.g., changes in heart rate and skin conductance level). Cue reactivity is thought to play an important role in maintaining drinking behaviour (Drummond, Glautier, Tiffany, \& Remington, 1995).

Schulze and Jones (2000) argue that alcohol cues may also lead to the activation of alcohol-related outcome expectancies. The notion that the activation of alcohol-related cognitions may be cue-reactive has also been proposed by Glautier and Spencer (1999). Comparing, light, moderate and heavy drinkers, they investigated whether alcohol-related cues could activate, or facilitate access to alcohol-related concepts stored in memory. Participants had to generate sentences with alcohol-ambiguous words. More alcohol-related sentences were produced when alcohol cues had been presented prior to the task. Further, heavy drinkers were more prone to interpret alcohol-ambiguous words as being alcohol- 
related. On the basis of these results, one coull argue that the activation of alcohol cognttions from memory can indeed be cue-reactive.

Since learning does not take place in a vacuum, but always against a specific array of background stimuli (e.g.y, environment, mood, and time), it is conceivable that contextual information also becomes encoded in memory during the learning of alcohol-related concepts, and hence may influence the accessibility of the alcohol-related memory network. Wall, McKee, Hinson and Goldstein (2001) observed that environmental context (a bar versus a laboratory setting) influenced expectations regarding the effects of alcohol. Participants held more positive alcohol outcome expectancies in the bar than in the laboratory (see also Wall, McKee, \& Hinson, 2000). Although these results indicate that contextual information affects the activation of alcohol-related cognitions, it is not clear how such contextual information is encoded in memory. Contemporary associative learning research has shown that context need not be encoded as a stimulus directly associated with a given outcome. An environmental context can come to control, or modulate, the activation of a specific association in memory (Bouton, 1993). With regard to alcohol drinking behaviour, a similar assumption was proposed by Drummond (2000), who suggested that a drinkingrelated environment may not serve as a cue directly eliciting alcohol cue reactivity, but merely affects reactivity indirectly by increasing the perceived salience of an explicit alcon hol-related cue.

In the present study, it was investigated whether alcohol-related concepts can be primed by the presentation of a verbal alcohol-related stimulus, and if so, whether the envirommental context in which the prime is presented affects the degree of primed access to alcohol-related cognitions. It was expected that such a prime would promote the accessibility of alcohol-related concepts, particularly when presented in a drinking-related context.

\section{Method}

\section{Paricipanrs}

A total of 70 participants took part in the experiment ( 13 men, 57 women). Most participants were first-year psychology students from Maastricht University. The participants had an mean age of $\| 9.8$ years $(S D=3.3$ ), and reported a mean consumption of 9 alcoholic drinks in the week prior to their participation in the experiment $(S D=9.6)$. Upon completion of the experiment, each participant was debriefed and received course credits, or $€ 7$.

\section{Procedure, Materials and Design}

Participants were randomly assigned to take part in the experiment in one of two environ. mental contexts. They were tested in groups containing 7 to 12 participants in either a pub-like, or an office-like setting. The pub was a dimly lit room at the psychology department building of the 
Maastricht University "measuring approximately 30 square meters. It contained a wooden floor, 5 small wooden tables, and 12 wooden chairs. A bar was placed at the left side of the room. On the left wall, behind the bar, were wooden shelves upon which stood bottles containing alcohol of different brands. Mirrors were placed against the hind wall of the room and on the remaining walls different alcohol advertising posters were hung. The office setting was a brightly lit room at the Mastricht University, measuring approximately 20 square meters that contained a green carpet floor, and a large, white table surrounded by 12 desk chairs. A filing cabinet stood against the hind wall and a large whiteboard was placed against the wall at the right from the exit.

Upon arrival, participants first received both verbal and written instructions and signed a consent form. Participants were told that the main purpose of the experiment was to qualitatively measure individual differences in creative writing. They were told that they would have to perform two sentence generation tasks. These tasks were largely based on the sentence generation task as described by Glautier and Spencer (1999). Both tasks consisted of 15 words that were read and spelled out loud one at a time by the experimenter. Immediately after each presented word, the participants had $30 \mathrm{sec}$ to write down a sentence containing the presented word. The first word of each task was either 'beer' (a specific alcohol-related word), or 'milk' (a nonalcohol-related word). Of the other 14 words, 4 words had an alcohol-ambiguous meaning and the other words were neutral fillers. The number of alcohol-related sentences gemerated with the ambiguous words served as the dependent variable. When the first word of the first sentence generation task had been 'beer', then 'milk' was the first word of the second task and vice versa.

After completion of the first sentence generation task, participants had to perform a spelling task. Each participant received a short text in which they had to indicate the spelling errors. The text did not hold any references to alcohol whatsoever and was intended to attenuate a potential carry-over effect of the semantic prime ('milk' or 'beer') presented on the first sentence generation task. After the spelling task, participants performed the second sentence generation task. Three independent raters separately classified the generated sentences as either alcohol-related or not, and the eventual classification was made on a majority rule basis (see also Glautier and Spencer, 1999).

After completing the second sentence generation task, each participant had to fill out a drinking history questionnaire on which they indicated the number of standard alcoholic drinks they had consumed during the week prior to their participation in the experiment. This questionnaire is largely based on the timeline follow back procedure (TLFB), which is frequently used in alcohol and drug research and has proven to be a reliable measure of assessing drinking behaviour (see Sobell, Sobell, Leo, \& Cancilla, 1988). Next, the participants were debriefed explaining the true purpose of the experiment, after which they received either course credits or payment.

\section{Results}

A verbal prime ("milk' versus 'beer") x context (pub versus office) analysis of covariance was conducted with the number of consumed units of alcohol in the week prior to testing 
as the covariate, and the number of generated alcohol-related sentences with the alcohol ambiguous words as the dependent variable.

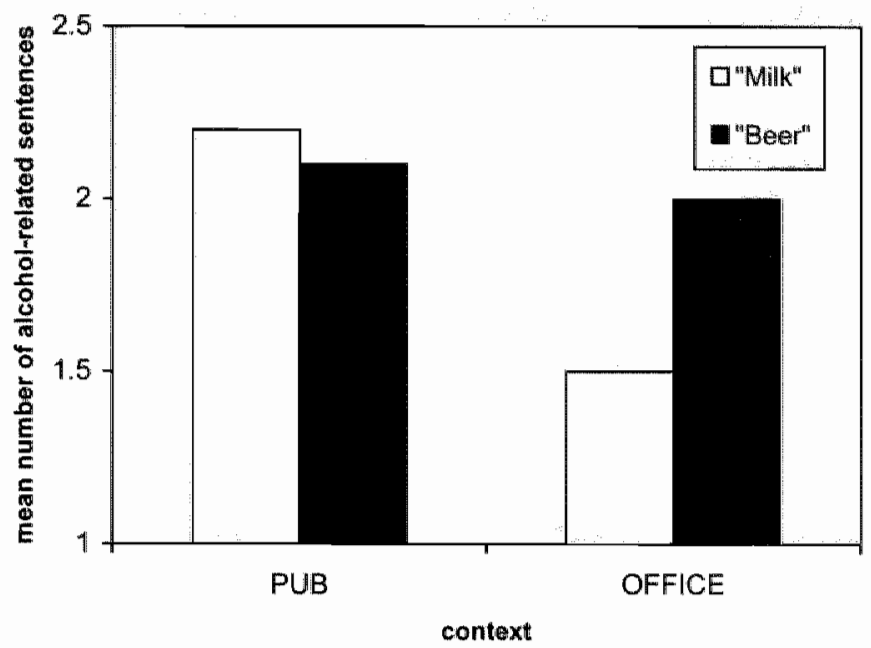

Figure 6.1. The mean number of alcohol-related sentences generated by the participants following the alcohol-related and the neutral verbal prime ('beer' versus 'milk'), for each context (pub versus affice).

The results are depicted in Figure 6.1, showing the mean number of alcohol-related sentences generated by the participants in the pub, or in the office, after having been presented the allcohol prime ('beer'), or the neutral prime ('milk').

A main effect of environmental context (pub versus office) was found, $F(1,67)=$ 7.56, $p<.01$, indicating that participants generated more alcohol-related sentences with the alcohol-ambiguous words in the pub than in the office. No main effect of the prime was found, $F(1,67)<1$. The covariate proved to be significant, $F(1,67)=4.68, p<05$, indicating that the more alcohol one had drunk in the week prior to testing, the more alcoholrelated sentences were generated. An interaction effect between the prime and context was found, $F(1,67)=4.17, p<.05$, indicating that the prime did have an effect on the activation of alcohol-related cognitions, but that this effect differs between the two groups.

Post hoc t-tests revealed that for the participants in group Pub, the prime ('milk' or 'beer") did not affect the interpretation of the alcohol-ambiguous words, $t=.68$, ns, whereas the word 'beer' did promote an alcohol-related interpretation of the ambiguous words in the participants tested in the office, $t=2.17, p<.05$. 


\section{Discussion}

It was hypothesised that environmental context would indirectly affect priming of alcoholrelated concepts stored in memory. No support for this hypothesis was found. Rather than controlling the degree of priming by a verbal alcohol stimulus, an alcohol-related environment directly appears to facilitate access to the alcohol-rellated memory network. Alcoholambiguous words were more easily interpreted as alcohol-related when presented in the pub than when presented in the office. These results provide further evidence for the view that the activation of alcohol-related concepts from menory can be facilitated through priming. The presentation of a verbal alcohol stimulius, exposure to an alcoholic drink (Glautier \& Spencer, 1999), and the exposure to a drinking-related setting can all promote the access to an alcohol memory network. The present results also demonstrate that the priming effect of these alcohol-related stimuli and contexts do not simply add up (see also Wiers, Havermans, \& Granzier, 2004). Further, the results extend previous findings that alcohol memory accessibility is affected by prior alcohol drinking experience, indicating that alcohol-related concepts are acquired and stored in memory in an associative manner.

Although the present findings appear to be quite straightforward, there is an alternative explanation for the apparent context dependent activation of alcohol-related concepts. A drinking-related environment, such as a pub, comprises a constellation of distinct alcohol-related stimuli. It is possible that not the entire alcohol-related context, but rather a single, specific feature of this environment promoted access to the alcohol-related meaning of the ambiguous words. It could well be that any other type of context that does not comprise such a constellation of specific alcohol cues (e.g., physiological state, mood, or time of day) will affect the priming of alcohol-related cognitions in a more indirect manner. Greeley and Ryan (1995) have suggested that a withdrawal state may function as an internal context signalling that drug-taking behaviour will be negatively reinforced. In other words, an internal contextual variable might control the meaning of drug-related cues and subsequent drug-taking behaviour. However, whether such contextual information will indirectly affect the extent to which a specific alcohol-related cue can activate alcohol cognitions from memory remains to be examined. Further, it should be noted that participants in the present study were either tested in an office-like or a pub-like environment. Both contexts were situated in the Maastricht University psychology department building. More outspoken, or even a different patten of results might have been found if participants had been tested in either a real pub or office.

It has been demonstrated that particularly positive alcohol outcome expectancies are more strongly activated in a drinking-related environment. The differential memory activation of alcohol-related cognitions across settings may provide an account for the observed contextual variability in alcohol consumption (see Wall, et al., 2001; Wiers, et al., 2004: Sher, 1985). The present findings provide support for this situational-specificity hy- 
pothesis. However, determining the exact relation between context-specific activation of alcohol-related cognitions, the motivation to consume alcohol and subsequent alcohol drinking behaviour requires further experimental testing. 


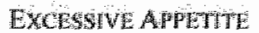




\section{PART II}

\section{THE PAVLOVIAN NATURE OF AN EXCESSIVE APPETITE FOR FOOD}

The most sincere love of all is the love of food. 
EXCESSIVE APPETTE 


\title{
CHAPTER 7
}

\section{OBESE WOMEN DEMONSTRATE STRONGER SENSORY-SPECIFIC SATIETY THAN NORMAL-WEIGHT WOMEN*}

\begin{abstract}
Sensory-specific satiety refers to a decrease in palatability of a food during consumption of this food as compared to other uneaten foods. Sensory-specific satiety is thought to play an important role in meal termination and the motivation to consume a varied meal. Therefore, several researchers have argued that sensory-specific satiety may also play an important rolle in the development and maintenance of obesity. It has been argued that the degree of experienced sensory-specific satiety in obese people differs from normal-weight people; that is, obese persons should display less sensory-specific satiety, thus promoting overeating. This hypothesis was tested in the present investigation comparing obese wersus normal-weight females. All participants reported a decrease in palatability of a drink, after being exposed to this drink for 15 minutes. However, only the obese participants demonstrated clear sensory-specific satiety. It is concluded that obese women may be more sensitive to specific sensory charicteristics of food than normal-weight women.
\end{abstract}

"This chapter is an adapted wersion of: Havermans, R.C., Hermans, L.., Roefs, A., Nederkoorn, C., dansen, A. (2004), Obese women demonstrate stronger sensory-specific satiety than normat-weight women Manuscript submitted for publication. 
When a food is eaten to siatiety, the pleasantness of the appearance, smell, taste and texture of that food decreases as compared to other uneaten food. This effect has been termed sensory-specific satiety (SS5; Rolls, 1986). It has been found that SSS already occurs during consumption of a meal and is strongest shortly after eating a food to satiety (Hetherington, Rolls, \& Burley, 1989). This implies that SSS does not depend on postingestive feedback. Indeed, energy content of a food has not been found to play an important role in SSS (Miller, Bell, Pelkman, Peters, \& Rolls, 2000; Bell, Roe \& Rolls, 2003). Further, although SSS is strongest after a food is eaten to satiety, it can also occur after eating a fixed preload (Hetherington \& Rolls, 1989), or even after repeatedly smelling or chewing a food (see Rolls \& Rolls, 1997). Therefore, SSS can be said to be the result of repeated sensory experience with a given food.

Although there is still debate concerning the precise mechanism underlying SSS, both animal and human studies indicate that habituation plays an important role (Swithers \& Hall, 1994; Hetherington \& Rolls, 1996). Not only pleasantness of a food decreases with repeated exposure to this food, but also the sallivary response to this particular food. When another food cue is presented salivation recovers, indicative of dishabituation (Epstein, Rodefer, Wisniewski, \& Caggiula, 1992). This is also true for eating behaviour. Eating is reinstated after having eaten a specific food to satiety when a novel food cue is presented (Wisniewski, Epstein \& Caggiula, 1992). This then implies that a varied meal should enhance eating behaviour, and several studies demonstrate that this is indeed the case (see e.g., Rolls, van Duijvenvoorde, \& Rolls, 1984).

The function of SSS then is i) promoting meal termination, hence preventing overeating (Hetherington, 1996), and ii) promoting the motivation to eat a more varied meal, thus preventing potential nutrient deficiencies (Rolls, 1986). As SSS plays an important role in eating behaviour, it has been suggested to play an equally important role in the development and maintenance of obesity. The degree of SSS is thought to differ between obese and nomal-weight persons. However, as of yet, it is unclear how SSS in obese persons differs. On the one hand, SSS can be thought to be less pronounced in obese persons, leading to overeating of a single meal. On the other hand, obese persons might demonstrate stronger SSS, enhancing the motivation to eat more varied and thus leading to overeating (see Raynor, \& Epstein, 2001). Very few evidence of such individual differences in SSS has been found. In a series of three experiments, Hetherington and Rolls (1989) found that anorexic and nondieting normal-weight control participants demonstrated SSS, whereas bulimic and overweight dieting participants did not. Obese persons also tend to show less rapid sallivary habituation to palatable food cues than normal-weight persons (Epstein, Paluch, \& Coleman, 1996). These results suggest that SSS may be less pronounced in obese persons. However, Spiegel and Stellar (1990) when comparing underweight, normalweight and obese participants, did not find any indication of a difference in SSS between the obese and normal-weight participants. A difficult matter in examining the role of SSS 
in obesity is that when instructed to eat a food to satiety, obese participants may tend to consume a larger amount than normal-weight participants, leading to a difference in sensory experience with the test food (Raynor, \& Epstein, 2001). Nevartheless, the few studies investigating individual differences in sensory-specific satiety do suggest that obese persons may demonstrate differential SSS; that is, demonstrating less SSS than normal-weight controls. This finding though requires further empirical validation. In the present investigation, it was examined whether obese persons indeed demonstrate less SSS than nomalweight persons, when the degree of sensory experience to the test food is controlled for.

\section{Method}

Table 7.1

Participant characteristics describing mean age, mean Body Mass Index (BMI" $\mathrm{kg} / \mathrm{m}^{2}$ ), mean score on the Restraint Scale, and mean score on the Beck Depression Inventory (BDI) for each group (normal-weight versus obese women).

\begin{tabular}{lrr}
\hline & \multicolumn{1}{c}{ Normal-weight } & \multicolumn{1}{c}{ Obese } \\
\cline { 2 - 3 }$N$ & 41 & 48 \\
Age (years) & $42.4(7.4)$ & $41.0(6.5)$ \\
BMI & $22.9(2.3)$ & $37.7(5.4)^{\star}$ \\
Restraint score & $9.9(4.1)$ & $19.4(5.2)^{\star}$ \\
BDI & $6.6(4.9)$ & $13.8(6.5)^{*}$ \\
\hline
\end{tabular}

Note. Values in parentheses represent the $S D$, and "indicates a significiant difference between the two groups with $p<.05$.

\section{Participants}

A total of 89 female participants were recruited via advertisements in a national Dutch women"s magazine and local newspapers. These women were part of a larger pool of participants who agreed to take part in a series of unrelated experiments at the psychology department of Maastricht University, one of which concerns the present investigation. Upon responding to the advertisement, participants received a set of questionnaires including the Beck. Depression Inwentory (BDI) which assesses the degree of depressive symptoms (Beck \& Beamesdorfer, 1974). On the basis of self-reported body mass index (BMI; $\mathrm{kg} / \mathrm{m}^{2}$ ) 48 participants were assigned to group Obese (BMI $>=30)$ and 41 participants served as normal-weight controls $(19<=\mathrm{BMI}<=25)$. All took part on a voluntary basis, but each participant was provided with a personalised fee to cover travel expenses. The experiment was approved by a local ethical committee at Maastricht University. Participant characteristics are displayed in Table 7.1. 


\section{Procedure, Materials, and Design}

The experimient was conducted between 3 and 4 PM. All participants had received the same lwnch (sandwiches) at around 12 noon at Maastricht University, and it was ensured that participants did not eat or drink anything 1 hr prior to their participation in the present study. Participants were tested in simall groups $(n=7-11)$ in a quiet research laboratory. On arrival, participants were invited to take a seat at any of 12 tables. The tables were placed against the walls of the laboratory and were divided by screens into separate compartments to prevent participants from directly communicating with one another during the experiment.

Participants received both verbal and written information concerning the procedure of the experiment. Next, they had to rate their appetite on several visual analogue scales (VASs). Questions concerning appetite were: "How hungry are you right now?", "How thirsty are you right now?" "How full are you right now?", "How nauseated do you feel right now?", and "How much food do you desire to eat right now?". These questions were followed by a 100-mm horizontal line anchored "not at all..." (e.g.,...hungry) on the left and "extremely..." (e.g., ... hungry) on the right. The anchors for the desire to eat question were "nothing at all" and "a large amount". Participants marked their responses on these 100-mm VASs (see also Bell, et al., 2003). Next, participants were served two transparent plastic cups: One containing $100 \mathrm{~m}$ of Roosvicee Dieet (De Ruijter, Netherlands; one part of fruit syrup mixed with 4 parts of water $(17 \mathrm{kcal} / 100 \mathrm{~g})$ ), and the other one containing $100 \mathrm{ml}$ of carbonated Lipton ice tea Light (Van den Bergh BV, Netherlands; $12 \mathrm{kcal} / 100 \mathrm{~g}$ ). Both drinks were served at room temperature. They were instructed to taste and evaluate the palatability of each drink, labelled A and B, on 5 different 100 mm VASs (taste test 1). Questions concerning palatability were based on the questions used by Bell, et al. (2003) and were: "How pleasant is the appearance of this drink right now? "How pleasant is the odour of this drink right now?", "How pleasant is the taste of this drink right now?", "How pleasant is the mouth feel of this drink right now?", and "How much of this drink would you like to drink right now?" (prospective consumption). The anchors of the first four VASs were "not at all pleasant" on the left and "extremely pleasant" on the right. For the last question, the anchors were "nothing at all" on the left and "a large amount" on the right. After this taste test, all participants received 2 transparent plastic cups witly both cups containing $250 \mathrm{~mL}$ of either the fruit syrup or ice tea (counterbalanced between participants). Raynor and Epstein (2001) have argued that the difficulty of determining differential SSS between obese and normal-weight participants is that obese persons are likely to consune a larger volume than normal-weight participants. This would make it difficult to ascertain whether a potential difference in SSS can be attributed to being obese or to any differences in sensory experience. Therefore, we did not instruct the participants to drink the test drink until satiety. Instead, each participant had to expose herself to the drink carefully following the instructions of the experimenter. During a period of 15 minutes, every $60 \mathrm{sec}$ the experimenter instructed the participants to take one of the two cups, to look at the drink for $10 \mathrm{sec}$, to sniff the drink for $10 \mathrm{sec}_{\text {, }}$ to take a sip and experience the mouth feel of the drink for $10 \mathrm{sec}$, and finally to swallow it to experience the full taste of the drink. Throughout this $15 \mathrm{~min}$ signalled exposure procedure, the experimenter monitored adherence to the instructions. After this exposure procedure, all cups were gathered and weighed to determine the amount of consumption of the test drink. At the post-test, all participants - similar to the pre-test - received two cups with one cup containing $100 \mathrm{ml}$ fruit syrup 
and the other $100 \mathrm{ml}$ ice tea, and were instructed to rate their appetite and palatability of the drinks for a second time (taste test 2 ).

After the second taste test, participants received a booklet containing 20 different full colour pictures of contemporary artwork and were instrueted to carefully look at each picture. Next, they received a scoring sheet and were asked to grade each work of art on a scale ranging from 0 ("very disliked") to 10 ("very liked"). This task had a total duration of approximately 20 minutes and served as a filler task. After completing the arts task, participants were instructed to tate their appetite and the palatability of drinks $A$ and B for a third and final time (taste test 3). Directly after finishing the experiment, weight and height of each participant was measured to verify selfreported BMI, and participants were asked to fill out the Restraint Scale that measures dietary restraint (Herman \& Polivy, 1980).

\section{Results}

Data from 4 participants in the normal-weight control group were excluded from the following analyses because their BMI (measured directly after the experiment) did not falt within the range 19-25. The data from 4 participants in group Obese were excluded from the following analyses because their BMI was below 30 .

Test drink consumption. The $15 \mathrm{~min}$ signalled exposure procedure was used to ensure that all participants had an equal amount of sensory experience with the test drink. However, although participants were instructed to consume one sip at a time of the test drink, the volume of consumption might still have differed between the two groups. The obese participants indeed tended to drink more than the normal-weight controls during the 15 min exposure ( $M$ difference $=33.19 \mathrm{~g}, S E M=18.12 \mathrm{~g}$ ), but this difference did not reach significance, $t(79 d f)=1.83, p=.07$.

\section{Appetite ratings}

Figure 7.1 displays the mean appetite ratings at each taste test for each group separately. To test whether appetite differed between the two groups at the start of the experiment, t-tests were conducted comparing the two groups on each appetite measure (i.e., hunger, thirst, fullness, nausea, and desire to eat) as rated directly prior to the first taste test. No significant differences in hunger, thirst, fullness, nausea and desire to eat were found, all $p s>.10$. 


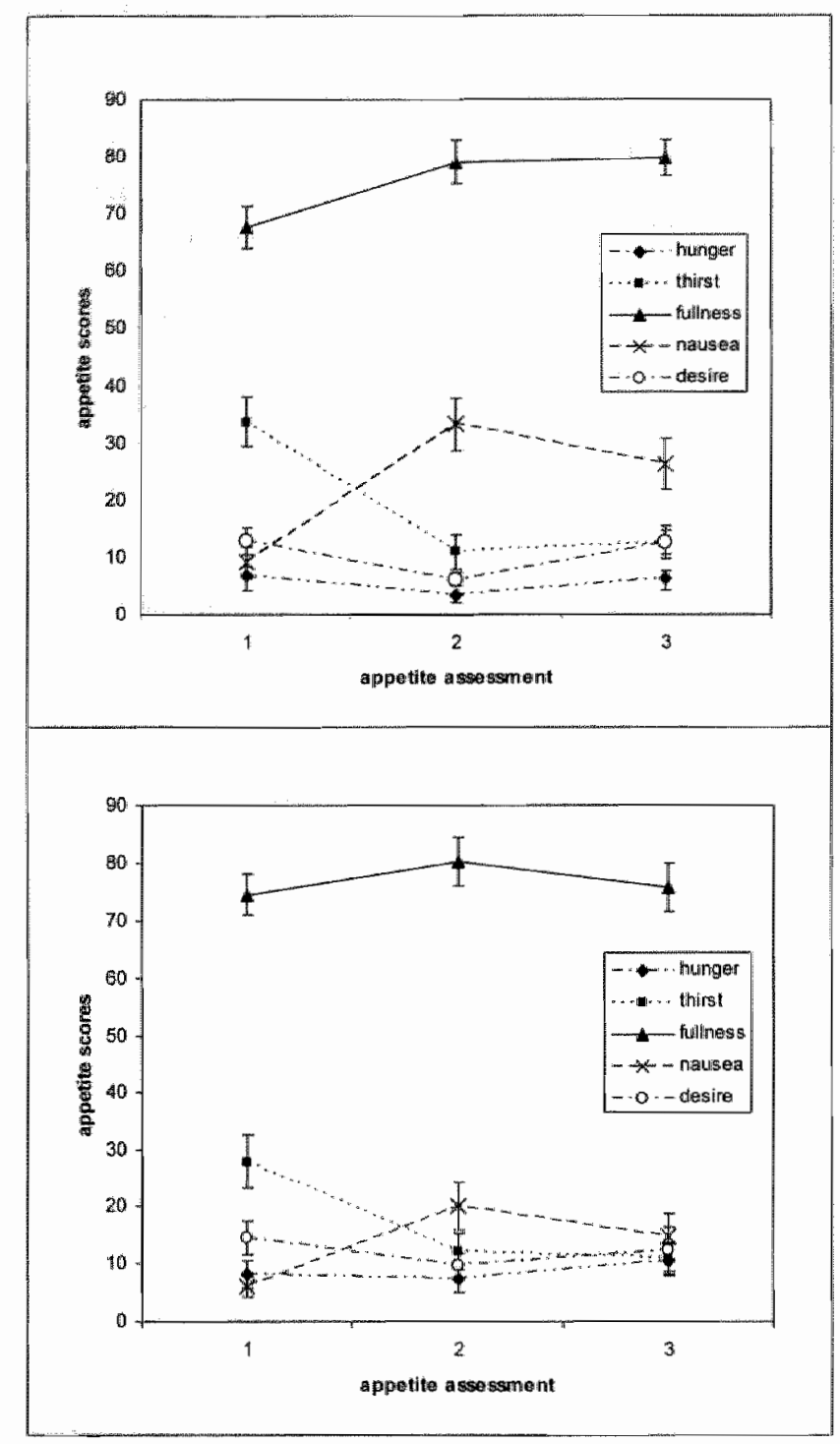

Figure 7.1. Mean appetite ratings (士 SEM) for each appetite assessment during the experiment. The top panel displays the appetite ratings for the normal-weight participants, and the lower panel displays these ratings for the obese participants.

To further test the time course of appetite ratings and whether this differed between groups, 3 (Taste test: 1, 2, or 3) x 2 (Group: obese versus normal-weight) analyses of variance (ANOVAs) were conducted for each of the 5 different appetite ratings. For ratings of hunger, a main effect of Taste test was found, mainly reflecting a decrease in hunger di- 
rectly after the 15 minute exposure to the test drink and subsequent increase in hunger after the 20 min filler task, $F(2,158)=5.64, p<.01$. No main effect of Group, or an interaction between Taste test and Group was found, $p s>10$. For ratings of thirst, similar effects were found. Thirst decreased markedly after the 15 min exposure, $F(2,158)=35.21, p<.001$. Group assignment did not affect thirst rating over time as evidenced from the absence of a main effect of Group and Group $x$ Taste test interaction, $p s>.10$. Ratings of fullness followed a similar pattern with a main effect of Taste test, $F(2,158)=4.72, p<.05$, and no effect of Group, or an interaction effect between Group and Taste test, ps $>.10$. All participants reported being more nauseated after the $15 \mathrm{~min}$ exposure to the test drink, $F(2$, $158)=28.18, p<.001$. In contrast with the other ratings of appetite, ratings of nausea did differ between groups, that is, the obese participants reported stronger nausea than the normal-weight participants, $F(1,79)=4.29, p<.05$. No interaction between Taste test and Group was found, $p>1.0$. Finally, for desire to eat, a strong effect of Taste test, $F(2,158)$ $=9.05, p<.001$, but no effect of Group, or a Group $\times$ Taste test interaction was found, $p \mathrm{~s}>$ .10 .

\section{Palatability ratings}

To determine whether the $15 \mathrm{~min}$ exposure to the test drink had led to negative changes in the palatability of this drink as compared to the control drink (i.e., SSS) and whether such an effect differed between the groups, repeated measures ANOVAs were conducted with Drink (the 15 min test drink versus the control drink), Taste test $(1,2$, or 3 ) as within-subject variables, and Group (obese versus normal-weight) as between-subjects variable for each separate palatability score (i.e., appearance, smell, taste, mouth feel, and prospective consumption). Scores on the Restraint Scale and BDI were not found to be significant covariates in any of the following analyses.

Figure 7.2 displays the difference in ratings of the $15 \mathrm{~min}$ exposure drink versus the control drink for each separate palatability measure at each taste test. A negative value refers to the test drink receiwing a lower palatability rating than the control drink. The top panel of Figure 7.2 displays these results for the normal-weight participants and the lower panel for the obese participants.

Appearance ratings. A main effect of Drink was found, $F(1,79)=5.64, p<.01$, indicating that the appearance of the control drink was rated significantly more pleasant than the appearance of the test drink. A significant Drink x Group interaction was found, $F(1,79)=6.10, p<.05$, reflecting that the effect of Drink was stronger in the obese group. Further, a main effect of taste test was found, $F(2,158)=27.62, p<.001$, primarily reflecting the strong decrease in pleasantness of appearance of both drinks after the 15 min exposure. A Drink $x$ Taste test interaction was found, indicative of SSS, $F(2,158)=7.32$, $p<.01$. Participants demonstrated a stronger decrease in pleasantness of appearance of the test drink than of the control drink. No further effects were found (all $p s>.10)$. 


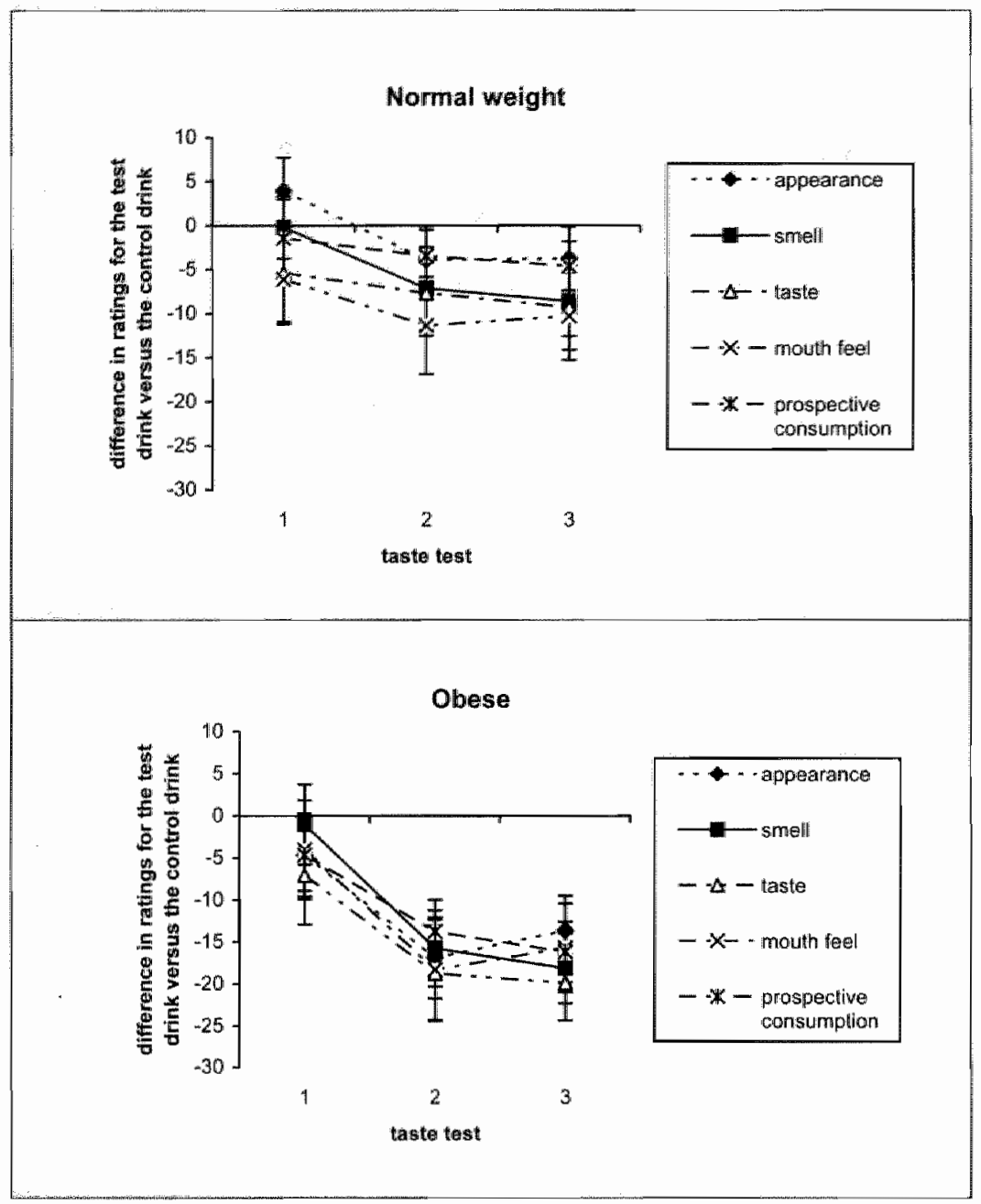

Figure 7.2. Mean difference in palatability ratings ( \pm SEM) between the test and the control drink for each taste test. The top panell displays these palatability ratings for the normal-weight participants, and the lower panel displays these ratings for the obese participants.

Contrary to our expectations, no three-way Drink $\times$ Taste test $\times$ Group interaction was found. However, considering the explicit a priori hypothesis that the degree of SSS differs between obese and normal-weight women, we examined the degree of SSS within each separate group, conducting a repeated measures ANOVA with Drink and Taste test as within-subject factors and appearance ratings as the dependent variable. A significant Drink $\mathrm{x}$ Taste test interaction was found within the obese group, $F(2,86)=6.13, p<.01$, but no such clear interaction effect was found within the normal-weight control group, $F(2$, 
$72)=2.07, p=.13$. In other words, contrary to the hypothesis, the obese women demonstrated SSS for the pleasantness of the appearance of the drinks, whereas the normalweight women did not:

Smell ratings. For ratings of the pleasantness of smell of the two drinks, a main effect of Drink was found, $F(1,79)=15.60, p<.001$. Further, a main effect of Taste test was found, $F(2,158)=25.53, p<.001$, primarily reflecting the decline in rated pleasantness of the smell of both drinks after the 15 min exposure to the test drink. A significant Taste test $\mathrm{x}$ Drink interaction indicative of SSS was found, $F(2,158)=7.76, p<.01$. The participants demonstrated a stronger decrease in ratings of pleasantness of smell for the test drink than for the control drink. No further effects were found (all $p s>.10$ ).

We examined within eacil group the degree of SSS regarding pleasantness of smell of the two drinks. Again, a significant Drink x Taste test interaction, indicative of SSS, was found within the obese group, $F(2,86)=6.24, p<.01$, but no strong evidence of SSS was found for the normal-weight participants, $F(2,72)=2.18, p=.12$.

Taste ratings. When analysing the ratings of pleasantness of taste, a similar pattem of results was found. A main effect of Drink was found, $F(1,79)=12.18, p<.01$. Ratings of pleasantness of taste declined across the consecutive taste tests, $F(2,158)=32.91, p<$ .001 . A significant Drink $x$ Taste test interaction indicative of SSS was found, $F(2,158)=$ $3.69, p<.05$. Ratings of pleasantmess of taste of the test drink deceased more than ratings of the taste of the control drink. No further effects were found (all $p \mathrm{~s}>.10$ ).

Again, the degree of SSS was investigated within each of the two groups. A near significant Drink $x$ Taste test interaction was found for the obese participants, $F(2,86)=$ $2.90, p=.06$, but no such an interaction was found for the normal-weight participants, $F(2$, 72) $=1.11, p=.34$.

Mouth feet ratings. Regarding the evaluation of the pleasantness of mouth feel of the two drinks, a main effect of Drink was found $F(1,79)=8.62, p<.01$, indicating that participants liked the mouth feel of the control drink better than the mouth feel of the 15 min exposure drink. A main effect of Taste test was found, $F(2,158)=33.36, p<.001$. Again, for the present ratings of mouth feel there was a strong indication of SSS reflected by a significant Drink $\times$ Taste test interaction, $F(2,158)=5.24, p<.01$. The pleasantness of the mouth feel of the control drink decreased less than the pleasantness of the mouth feel of the test drink. No further effects were found (all $p \mathrm{~s}>.10$ ).

The degree of SSS for mouth feel ratings was examined for each group separately. A significant Drink $x$ Taste test interaction was found for the obese participants, $F(2,86)=$ $4.49, p<.05$, but not for the normal-weight participants, $F(2,72)=1.33, p=.27$. Considering mouth feel ratings, again the obese women demonstrated SSS, whereas the normalweight participants did not.

Prospective consumption ratings. A main effect of Drink was found, $F(1,79)=$ $8.33, p<.05$. Participants indicated wanting to drink more of the control drink than the test 
drink. A significant Drimk $*$ Group interaction was found, $F(1,79)=3.83, p=.05$, reflecting that the effect of Drink tended to be larger in the obese group. This means that particularly the obese participants indicated wanting to drink more of the control drink than the test drink. Further, a main effect of Taste test was found, $F(2,158)=54.49, p<.001$, indicating that the wish to drink the two drinks in the near future declined across taste tests. A near significant Taste test $\times$ Group interaction was found, $F(2,158)=2.67, p=.07$, reflecting the larger decrease in prospective consumption ratings across taste tests for the obese participants. As for the other palatability measures, a significant Taste test $x$ Drink interaction was found, $F(2,158)=3.80, p<.05$, indicative of an overall SSS effect. No further effects were found (all $p s>.10$ ).

Additional analyses were conducted for each separate group to determine the degree of SSS within each group. Again, within the obese group a near significant Taste test x Drink interaction was found, $F(2,86)=2.84, p=.06$, indicative of SSS, but not within the normal-weight control group, $F(2,72)=1.20, p=.31$.

\section{Discussion}

In the present study, it was investigated whether obese women demonstrate less SSS aS compared to normal-weight women. Contrary to this hypothesis, it was found that obese women demonstrated SSS for all the different palatability measures (i.e., appearance, smell, taste, mouth feel and prospective consumption), whereas the normal-weight participants did not demonstrate such a clear SSS effect. Although both groups demonstrated a decrease in palatability ratings of the test drink, only the normal-weight women reported a near equal decline in palatability of the control drink. Furthermore, the obese women tended to show a stronger reduction in appetite after the 15 minute taste exposure than the normal-weight women; that is, the obese women reported a stronger increase in nausea after the 15 minute exposure than the normal-weight women. These results imply that there are differences in SSS between normal-weight and obese women. More specifically, obese women tend to show more pronounced SSS than normal-weight women.

The absence of a clear SSS effect in the normal-weight women can be attributed to the relative sensory similarity between the test and control drink. These drinks are both water-based liquids and thus have somewhat similar texture. Both drinks were also served at the same temperature and were both sweet-tasting. Such generalisation of SSS to other food sharing one or more sensory characteristics with the test food has been frequently demonstrated (see e.g., Rolls, et al., 1984; Guinard, \& Brun, 1998). The present difference in SSS between obese and normal-weight women can thus be attributed to obese women showing less generalisation of SSS, rather than showing a stronger decrease in palatability 
of the test food per se. It may be that obese women are more sensitive to specific taiste characteristics and as such, they may be inclined to demonstrate less generalisation of SSS than normal-weight women. This notion however, requires further research as in the present study only the palatability ratings of two beverages were compared, instead of a larger range of different food servings as is usually the case in studies investigating SSS.

Although it is argued here that the present findings can be ascribed to individual differences in sensory sensitivity, other factors may have played a role in the present results. Despite ensuring that participants had an equal amount of sensory experience with the test drink (i.e., equal amount of time spent looking and smelling at the drink and an equal amount of sips taken from the drink), the obese participants did tend to take bigger sips than the normal-weight controls. One could thus argue that the groups did have differential sensory experience with the test drink, leading to the present pattern of results. This is unlikely, however, as the mean difference in consumption comprised 33.19 grams (approximately $30 \mathrm{ml}$ ), which means that on average the obese participants consumed merely $2 \mathrm{ml}$ of the test drink per sip more than the normal-weight participants. However, the two groups also differed on characteristics such as depressive symptoms and restraint-status. The obese participants were sigmificantly more depressed and restrained than the normalweight participants. These factors may have confounded the present results. However, previous studies have demonstrated that both restraint-status and BDI scores do not significantly affect the magnitude of SSS (Tepper, 1992; Bell et al., 2003). Moreover, both scores on the Restraint Scale and BDI were not significant covariates in the analyses of the present study. Therefore, it is unlikely that differences in restraint-status and depressive symptoms between the two groups have played an important role in the present finding.

The present results then points to an important role of SSS in the devellopment and maintenance of obesity. As argued by Raynor and Epstein (2001), more pronounced SSS may motivate one to eat a more varied diet. Within an obesogenic environment where food of high caloric density is plentiful, inexpensive and available in many different varieties (see e.g., Hill \& Peters, 1998; Wadden, Brownell, \& Foster, 2002), stronger SSS may promote the development of obesity. People who generally experience strong SSS may be more motivated to eat a more varied meal as such variation attenuates SSS. When such variation comprises mainly high caloric food items, one will be in more danger of developing overweight and becoming obese. Indeed, McCrory et al. (1999) found that the increased prevalence of obesity is associated with an increase in the variety of the consumption of energy-dense food items.

In sum, in the present study obese women showed stronger SSS than nomalweight women. This difference may be attributed to the obese participants demonstrating less generalisation of a decrease in hedonics from the test drink to the control drink than the normal-weight control participants. Whether obese persons are truly less prone to dem- 
ExCLESIV1 APPETITE

onsitrate generalisation of $\mathrm{S} S \mathrm{~S}$ than nomal-weight persons requires further empirical validation. 


\title{
CHAPTER 8
}

\section{COMPARING CARBOHYDRATES AND DIETARY FAT IN CONDITIONING FLAVOUR PREFERENCES IN HUMANS*}

\begin{abstract}
A within-subjects design was used to determine whether a positive shift in flavour preference due to flavour-nutrient learning would be particularly large for flavours paired with a highcarbohydrate content. On a pre-test, each participant had to rate and rank order 7 distinct flavours of yoghurt on pleasantness and preference. During a total of 12 subsequent conditioning sessions, 3 of these flawours were paired with either a low-energy yoghurt, a carbohydrateenriched yoghurt, or a fat-enriched yoghurt. On a post-test, it was found that preference and liking for the high-carbohydrate paired flavour had increased, in contrast with the high-fat flavour and the flavour associated with the low-encrgy yoghurt. It is concludled that these results may reflect a discrepancy in the reinforcing potency of fat and carbohydrates.
\end{abstract}

\footnotetext{
"This chapter is an adapted version of: Havermans, R.C., Roefs, A., Lataster, T, Houben, K., \& Jansen, A. (2004). Comparing carbohydrates and dietary fat in conditioning flavour preferences in humans. Manuscript submitted for publication.
} 
The appreciation of the flavour of a food is largely based on experience. For instance, the mere repeated exposure to a food, or simply observing a significant other consuming a particular food will affect the preference for that food (see Jansen \& Tenney, 2001). Further, flavour-flawour learning can also lead to changes in perceived palatability of a food. For example, pairing a neutral llavour with an already highly preferred flavour (e.g., sweetness) will increase liking for the initially neutral flavour. Next to these effects, the postingestive consequences of food consumption also contribute to the acquisition of likes and dislikes for food (Capaldi, 1996).

Negative consequences of food consumption will lead to an aversion for the flavour of that particular food. For example, Garcia and Koelling (1966) showed that rats readily learn an aversion for a food when consumption of that food is followed by illness. Conversely, Bolles, Hayward, and Crandall (1981) found that rats learn an apparent flavour preference for food paired with high caloric density, suggesting that a positive postingestive consequence leads to a positive shift in the hedonic value of the flavour of the food.

Such flavour preference learning is attributed to Pavlovian conditioning. In Pavlovian conditioning, the probability of a response to a conditioned stimulus (CS) increases due to repeated pairings of the $\mathrm{CS}$ with a biologically relevant unconditioned stimulus (US). Pavlov (1927) demonstrated that dogs would come to salivate to a tone, after pairings of the tone with the presentation of food. Flavour preference learning is a form of conditioning where the preference for an initially neutral, or even disliked lavour increases due to pairing of the flavour of a food (the flavour CS) with the postingestive consequences of the nutrients in that food (the nutrient US) (Capaldi, 1996). Although it appears that a change in hedonic evaluation of the initially neutral flavour underlies flavour preference learning, recent animal studies have shown that flavour preference leaming is not necessarily accompanied by a change in hedonic evaluation (Myers \& Sclafani, 2003).

Positive shifts in flavour preference due to such flavour-nutrient learning has repeatedly been demonstrated in animals, using nutrient USs such as ethanol, fat, protein and carbohydrates (see Fedorchak, 1997). Conditioning of flawour preferences has also been demonstrated in humans. Booth, Mather, and Fuller (1982) found that adults readily learned a preference for food associated with a high-starch content. Birch, McPhee, Steinberg, and Sullivan (1990) demonstrated conditioned flavour preferences in children for drinks paired with a high-carbohydrate content. Similar results were found in children when pairing flavours with high dietary fat (Johnson, McPhee, \& Birch, 1991; Kern, McPhee, Johnson, Fisher, \& Birch, 1993).

In general, it is assumed that the conditioning of flavour preferences depends solely on the caloric density of the consumed flavour (see Birch, \& Fisher, 1996). This implies that different nutrients provide a common energy-related signal that reinforces the flavour, hence leading to the formation of a flavour-nutrient association. Although there is some support for this view (e.g., Mehiel \& Bolles, 1988; Pérez, Lucas, \& Sclafani, 1995), it 
downplays the differences between macronutrients. Not only do macronutrients differ in their caloric density (e.g., fat provides 9 kilocalories per gram, whereas carbohydrates and proteins deliver 4 kilocalories per gram), they are also metabolised at different rates. Further, the metabolism of different nutrients occurs in different tissues and organs, and nutrients may have different satiating effects (Blundell, Lawton, Cotton, \& Macdiarmid, 1996). Each of these factors can affect the timing, and the perceived intensity of the postingestive signal provided by macronutrients, and hence the learning of a flavour preference.

Sclafani (1990), in reviewing several studies on flavour-nutrient learning, reported that fat admimistrations may be less effective in conditioning flavour preferences in rats than the isocaloric administration of carbohydrates. Lucas and Sclafani (1999a, 1999b) directly compared the reinforcing and satiating effects of carbohydrate and fat in conditioning flavour preferences in rats. Carbohydrate proved to be more effective in conditioning a flavour preference than fat. Lucas and Sclafani (1999a) also demonstrated that carbohydrates have stronger satiating effects than fat. This may explain the differential reinforcing potency of carbohydrate and fat in conditioning flavour preferences in rats.

Several studies in human subjects also appear to suggest that fat may be less satiating than carbohydrates (see Blundell, et al., 1996). When one assumes that the reinforcing effect of nutrients in conditioning flavour preferences is largely determined by their satiating effects, this leads to the prediction that a conditioned flavour preference is more easily acquired when a flavour is paired with a high-carbohydrate content, rather than a highdietary lat content, when controlling for caloric density. Although dietary fat has been demonstrated to successfully condition preferences for flavours in humans (Birch, \& Fisher, 1996), the potentially differing effectiveness in conditioning such a preference between carbohydrate and fat has not been investigated in humans as of yet.

In the present study, the question whether carbohydrates are a more potent reinforcer than dietary fat in conditioning 1lavour preferences was investigated in human adults. Participants had to consume distinct flavours consistently paired with ather a low caloric density, a high-fat content, or a high-carbohydrate content. It was predicted that any positive shift in flavour preference would be particularly large for the flavour paited with the high-carbohydrate content.

\section{Method}

\section{Participants}

Eighteen females with a mean age of 19.1 years $(S D=0.8)$ took part in the experiment. $A$ total of 24 participants were originally recruited, but 3 candidate participants withdrew just before the start of the experiment, and another 3 participants were excluded for not attending to one or 
more conditioning sessions. Participants were told that the primary question of the study concemed Whether specific food components would affect cognitive performance. All participants were paid $€$ 50 and in addition received course credits upon completion of the experiment.

\section{Procedure, materials and design}

A within-subject design was used to investigate the question whether carbohydrates and dietary fat differ in their potential to condition a flawour preference. A local ethical conmittee ap. proved the research protocol. Participants were given a brief written description of the procedure before the start of the experiment and signed a consent form on the basis of this information. Participants were told that they had to regularly consume different yoghurt drinks in morning sessions between 9 and $12 \mathrm{AM}$ on 14 consecutive weekdays, after which they would receive a cognitive task in determining whether the consumption of the yoghurt drinks had affected their cognitive capacity. Participants were instructed not to have eaten anything prior to each session, thus being overnight food deprived at each session.

Seven different noncommercially available flavours of yoghurt were prepared by adding 3 $\mathrm{ml} / \mathrm{l}$ of flavouring extracts (Quest International BV, the Netherlands) to the yoghurt (Milsa light yoghurt, Frabelec, Belgium). The flavours were: melon, apple fig, coconut, mango, grape, and litchi. On a pre-test, all 7 distinct flavours of yoghurt were presented in $50 \mathrm{~g}$ servings each. These yoghurt servings were sweetened by adding $0.5 \mathrm{~g}$ of aspartame per $100 \mathrm{~g}$. On this test, each participant had to taste and subsequently rate the 7 different flavours on pleasantmess. Participants were instructed to consume as much of the different flavours as required to be able to adequately evaluate the flavours. Rating of the hedonic value of the flavours occurred on 11-point Likert scales ranging from -5 "very disliked" to +5 "very liked". Next, they had to indicate their flavour preference by ranking the flavours from most liked (value of I) to least liked (value of 7).

Flavour conditioning began the day after the pre-test. Different versions of yoghurt drinks were prepared: a low-energy, a high-carbohydrate, and a high-fat yoghurt. The low-energy version (CS-) was prepared with the light yoghurt, providing $33 \mathrm{kcal}(0.1 \mathrm{~g}$ fat, $3.4 \mathrm{~g}$ proteins, and $4.7 \mathrm{~g}$ carbohydrates) per $100 \mathrm{~g}$. The high-carbohydrate version ( $\mathrm{CS}+\mathrm{CHO}$ ) was prepared by adding $21 \mathrm{~g}$ of glucose (Cerestar, the Netherlands) to $79 \mathrm{~g}$ of the light yoghurt, providing approximately 110 kcal $(0.1 \mathrm{~g}$ fat, $2.7 \mathrm{~g}$ proteins, and $24.7 \mathrm{~g}$ carbohydrates) per $100 \mathrm{~g}$. The thigh-fat yoghurt (CS+FAT) was prepared by adding $9 \mathrm{~g}$ of suntlower wil (Amphora, the Netherlands) to $91 \mathrm{~g}$ of the light you ghurt, also providing approximately $110 \mathrm{kcal}(9.1 \mathrm{~g}$ fat, $3.1 \mathrm{~g}$ proteins, and $4.3 \mathrm{~g}$ carbohydrates $)$ per $100 \mathrm{~g}$. All yoghut versions were initially sweetened with $0.5 \mathrm{~g}$ aspartame per $100 \mathrm{~g}$ yoghurt. The different versions of yoghurt drinks had similar texture and mouth feel based on pilot testing. In this pilot study, participants $(n=10)$ had to consume $50 \mathrm{~g}$ of each yoghurt version, and were asked to report whether they could detect any flawour differences regarding mouth feel, texture, and sweetness. None of the participants reported tasting any difference in mouth feel and texture, but 6 out of the 10 participants reported finding the taste of the high-carbohydrate yoghurt a bit sweeter than the other two versions of yoghurt. This can be ascribed to glucose having a mild sweet taste of its own. Therefore, only half of the amount of aspartame (i.e., $0.25 \mathrm{~g}$ per $100 \mathrm{~g}$ yoghurt) was added to the high-carbohydrate yoghurt within the present experiment.

The 3 thavours with a ranking of 3,4 , or 5 at the pre-test were selected for each participant for presentation in the following conditioning sessions, ensuring that these flavours were neither 
very liked nor disliked. Flavour-nutrient pairings were counterbalanced between participants, with each of the three selected flavours being consistently paired with one specific version of the yo. ghurt (low-energy, high-carbohydrate, or high-fat), so that on average, the flavour of each version of yoghurt during conditioning sessions was equally liked, or preferred at the pre-test. The conditioning sessions were conducted across 12 consecutive weekdays, presenting each flavour-nutrient pairing 4 times in a random order for each separate participant.

On each conditioning session, a single $300 \mathrm{~g}$ serving of a specific navour-nutrient pairing (CS-, CS+CHO, or CS+FAT) was presented. All yoghurts were chilled to approximately 6 centigrade and served in $500 \mathrm{ml}$ paper cups sealed with a plastic lid. Since oil has the tendency to partially separate from the yoghurt, each yoghurt version was firmly stirred before serving. Participants drank the yoghurt through a straw that was inserted through the plastic lid.

A post-test followed the day after the last conditioning session and was identical to the pretest (no oil or glucose was added to the yoghurt at these tests). After the post-test, each participant received $€ 50$ and course credits and was fully debriefed about the true purpose of the experiment.

\section{Data analysis}

Preference data for CS-, CS+FAT, and CS+CHO, as indicated by the ranking of these flavours before and after conditioning, were compared using repeated measures analyses and t-tests. The same analyses were conducted comparing the hedonic ratings of the flavours before and after conditioning. All analyses were conducted using a rejection criterion $\alpha=.05$.

\section{Results}

Due to the fact that 6 of the initially 24 recruited participants had either withdrawn, or had been excluded, counterbalancing of the flavour-nutrient pairings was incomplete. An analysis of wariance (ANOVA) was conducted comparing the pre-conditioning rankings of each flavour-nutrient pairing (CS-, CS+FAT, or CS+CHO). No difference in ranking of the flavours was found $F(2,16)<1$, indicating there were no pre-conditioning differences in preferences for the selected flavours presented to each participant during subsequent condi. tioning sessions. A similar analysis was conducted, comparing the pre-conditioning hedonic values of the flavour-nutrient pairings. No differences in ratings were found $F(2,16)$ $=1.71, n s^{5}$.

Figure 8.1 displays the mean shift in preference for each flavour-nutrient pairing, comparing the ranking of the selected flavours before and after conditioning. A repeated measures ANOVA was conducted with time (pre-test versus post-test) and flavour-nutrient pairing (CS-, CS-FAT, or CS+CHO) as within-subject factors and the ranking of these flavours on the pre- and post-conditioning tests as the dependent variable. A main effect of time was found, $F(1,17)=6.12, p<.05$, indicating a general increase in preference for the flavours after conditioning. No main effect of flavour-nutrient pairing was found, $F(2,16)$ 
$=1.90, n s$. There was, however, a significant interaction between time and flavour-nutrient pairing, $F(2,16)=8.75, p<.01$, reflecting a nutrient-specific positive shift in flavour preference.

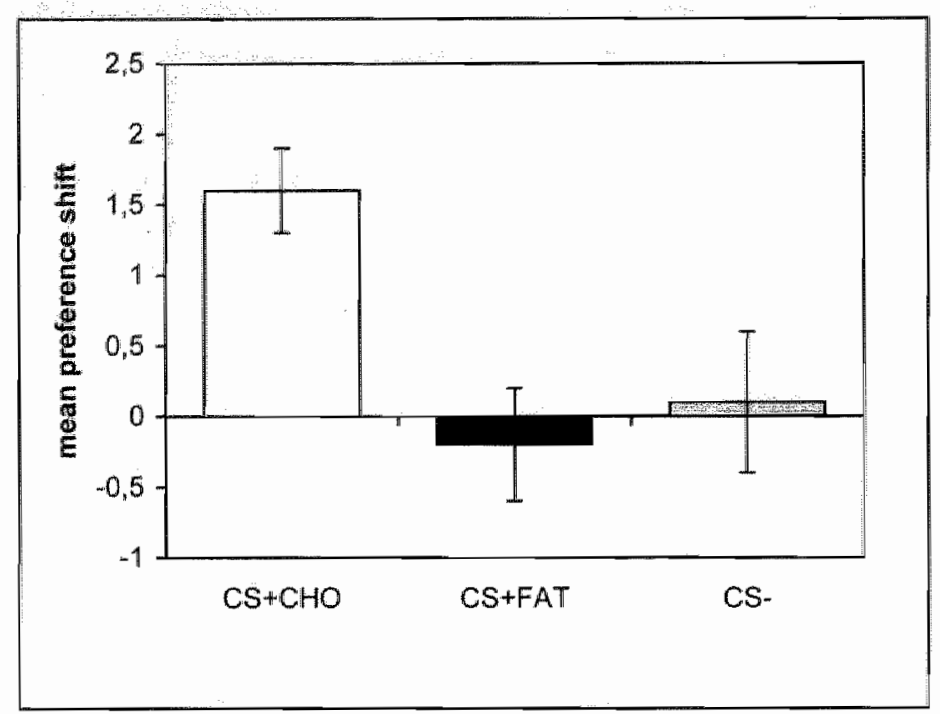

Figure 8.1. The mean shift in preference for each flavour nutrient pairing comparing the ranking of the selected flavour CSs before and after conditioning, with the error-bars representing the standard errors of the mean (SEM). CS+CHO refers to the flavour paired with the high-carbohydrate content; CS+FAT represents the flavour paired with the high-fat content; CS- is the flavour with no extra calories added.

Subsequent $t$-tests, revealed that participants ranked the carbohydrate-enriched flavour higher after conditioning, $(17)=4.12, p<.01$, whereas no changes in ranking were found for the flavour associated with the high-dietary fat, $f(17)=.57$, ns, and the lowenergy flavour, $t(17)={ }_{n} 78$, $n$ s. At the post-test, half of the participants preferred the flavour that had been consistently paired with the high-carbohydrate content to both the lowenergy and the high-dietary fat rellated flavour.

Figure 8.2 displays the mean shift in hedonic value for each flavour-nutrient pairing, comparing the ratings of pleasantness before and after conditioning. The same repeated measures ANOVA was conducted with the hedonic ratings, as indicated on the 11 point Likert scales before and after conditioning, as the dependent variable. No main effect of time was found, $F(1,17)=1.84, n s$. No main effect of flavour-nutrient pairing was found, $F(2,1.6)=1.25$, ns. There was a significant interaction between time and flavournutrient pairing, $F(2,16)=4.71, p<.05$, indicating a nutrient-specific increase in liking for a flavour. 
Subsequent $t$-tests revealed that participants liked the carbohydrate-enriched thvour better at post-test; $t(17)=2.61, p<.05$. No such positive shifts in hedonic value were found for the flavour associated with the high-fat content, $t(17)=.23$, and the low-energy flavour, $t(17)=1.00, n s$.

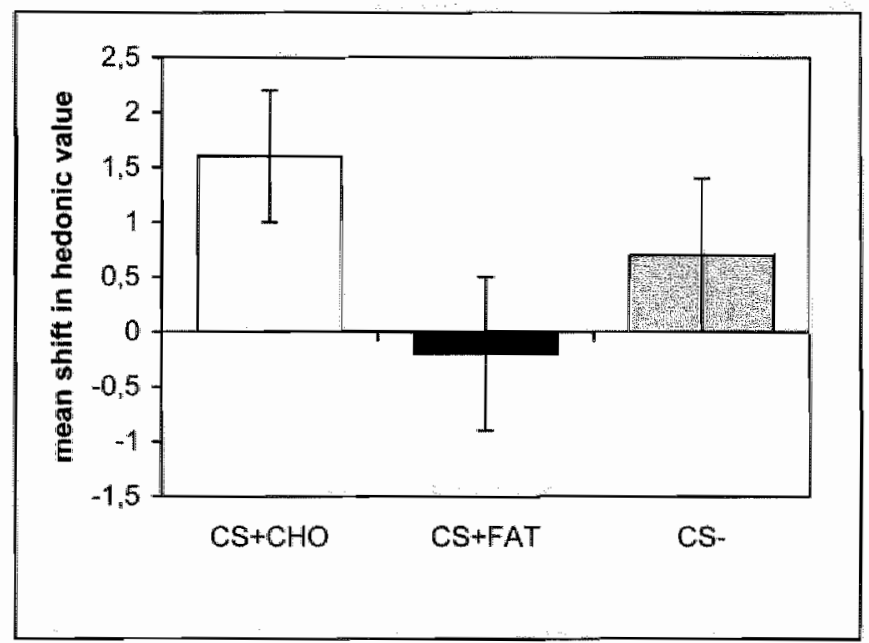

Figure 8.2. The mean shift in hedonic ratings for each flavour-nutrient pairing comparing rated liking of the selected flavours before and after conditioning, with the error-bars representing the SEMS. CS $+\mathrm{CHO}$ represents the flawour paired with the high-carbohydrate content; CS+FAT is the flavour paired with the high-fat content; CS- refers to the flavour with no extra calories added

\section{Discussion}

It was bypothesised that carbohydrates are a more potent caloric reinforcen than dietary fat in conditioning flavour preferences in humans. The results of the present study provide support for this hypothesis, demonstrating an increase in preference and liking for a flavour associated with a high-carbohydrate content, but not for a high-fat related flavour, despite their isocaloric density. These results extend the findings from several recent animal studies demonstrating differential reinforcing properties of fat and carbohydrates in conditioning flavour preferences (Lucas \& Sclafani, 1999a, 1999b).

The present results can be explained in terms of differential satiating effects of carbohydrate and fat. Results from several animal and human studies appear to suggest that carbohydrates have a more satiating effect than fat (see Blundell, et al., 1996; Lucas \& Sclafani, 1999a). However, it should be noted that these findings have not gone unchallenged. Several studies have found no such differential satiating effects of fat and carbohy 
drates when palatability and energy density was controlled for (see Rolls; \& Bell, 1999). Moreover, explaining the present results by claiming that carbohydrates provide stronger satiety implies that the flavour-reinforcing effects of nutrients directly reflect the degree of satiety they provide. Sclafani (1997), however, argues that to some extent the satiating and reinforcing effects of nutrients are independent.

An alternative explanation of the present results may be that glucose is absorbed and utilised more rapidly than triglycerides, as has been suggested by Lucas and Sclafani (1999b). In that case, the postingestive reinforcing signal provided by glucose may have been more contiguous with the flavour of the yoghurt than the postingestive signall provided by the high-dietary fat. Contiguity refers to the principle that associations are formed when two events occur together in space and time. The more contiguous two events are, the more rapidly an association is formed. Differential contiguity between the flavour and the experienced postingestive effects of fat and carbohydrate may thus have led to the observed difference in conditioned flavour preferences. This explanation implies that conditioning flawour preferences using fat as the nutrient US requires more intense conditioning. This is precisely what Lucas and Sclafani (1999b) found. When rats underwent conditioning sessions of 22 hours per day (instead of 30 minutes) they even showed stronger preferences for flavours paired with the administration of fat than for flavours paired with the administration of carbohydrates. More research is required to test the validity of explaining the present results in terms of differential metabolic rates of nutrients. For example, one could compare more slowly processed carbohydrates (such as starch) with dietary fat on their efficacy in conditioning a flavour preference, as was suggested by Lucas and Sclafani (1999b).

It should be noted that the present results are not necessarily the result of differential reinforcing potency of carbohydrates and dietary fat. A viable alternative explanation is that the present results reflect flavour-flavour learning. Although we attempted to control for differences in flavour characteristics between the three yoghurt versions, potential differences in palatability were not explicitly controlled for. Therefore, it is possible that the carbohydrate-enriched yoghurt might have been evaluated as much more palatable than the fat and low-caloric yoghurt version, and that the positive hedonic value of the carbohydrate-enriched yoghurt transfered to the flavour being paired with this particular yoghurt version.

In sum, the present results suggest that nutrients, when controlling for caloric density, may differ in their effect on conditioning flavour preferences in humans. This implies that the reinforcing potency of nutrients is not directly related to their caloric density. However, it should be noted that the present results can allso be interpreted in terms of flavour-flavour learning. The present results nevertheless warrant further research investigating the questions whether nutrients indeed have differential reinforcing potency and if so, to what degree this reflects the differential satiating properties of nutrients. 


\title{
CHAPTER 9
}

\section{STIMULUS GENERALISATION AND CONFIGURAL LEARNING AS A MODEL OF EVALUATIVE CONDITIONING*}

\begin{abstract}
Evaluative conditioning refers to the transfer of affective value to an initially neutral stimulas by pairing the neutral stimulus with an affective stimulus (positive, or negative). In the first part of this chapter the associative nature of evaluative conditioning is discussed and existing models of evaluative learning are briefly described. It is argued that these models of evaluative conditioning do not provide a complete account of evaluative conditioning and generate nonspecific predictions. The second part of the chapter concerns the development of a more specific model of evaluative conditioning, describing such conditioning in terms of Pearce's 1987 model of stimulus general isation and configural learning. By viewing the transfer of affect as a generalisation and configural learning process, this model can account for most denonstrations of evaluative conditioning and generates more precise and unambiguous prediclions.
\end{abstract}

\footnotetext{
"This chapter is an adapted version of: Havermans, R.C., \& Jansen, A. (2004). Stinnulus generalization and configural learning as a model of evaluative condinioning. Manuscript submitted for publication.
} 
Since preferences and aversions are important determinants of human behaviour, much research has been devoted in understanding the nature of human likes and dislikes. Some preferences and aversions are innate, such as the preference for sweet tastes and the aversilon for bitter tastes. However, most preferences and aversions are acquired with experience (Capaldi, 1996). Associative learning has been proposed as the primary mechanism underlying such devellopment of likes and dislikes (De Houwer, Thomas, \& Baeyens, 2001). In 1975, Levey and Martin demonstrated associatively acquired affect. In this seminal study they first let participants categorise pictures of paintings as liked, neutral, or disliked. In the subsequent conditioning phase, neutral pictures were consistently paired with either a liked, disliked, or another neutral picture. At test, where participants had to evaluate the pictures that had been presented during conditioning, it was found that the neutral pictures paired with the disliked stimuli were evaluated as more negative. Similarly, the neutral pictures paired with the liked pictures were now evaluated as more positive. Martin and Levey (1978) coined the term evaluative conditioning (EC) in referring to this apparent associative learning of preferences and aversions.

EC appears to be al reliable effect. Numerous picture-picture studies have successfully demonstrated transfer of affect, using pictures of fountains and sculptures (e.g., Hammerl \& Grabitz, 1993), or pictures of human faces (e.g., Baeyens, Eelen, Van den Bergh, \& Crombez, 1989). Apart from being a reliable effect, EC also appears to be a general phenomenon. The transfer of affect has been shown to occur using different flavours as stimuli (e.g., Zellner, Rozin, Aron, \& Kulish, 1983; Baeyens, Eelen, Van den Bergh, \& Crombez, 1990), or haptic stimuli (Hammerl \& Grabitz, 2000). Several studies have also demonstrated cross-modal EC (see e.g., Todrank, Byrnes, Wrzesniewski, \& Rozin, 1995). It should be noted though that cross-modal $\mathbb{E C}$ appears to be somewhat less robust than $\mathrm{EC}$ using stimuli of the same perceptual modality, as demonstrated by several failures to obtain cross-modal EC (see Rozin, Wrzesniewski, \& Byrnes, 1998).

Although EC has been studied extensively over the last three decades there is still considerable debate concerning the associative nature of the learning of likes and dislikes. In this chapter, I briefly summarise different demonstrations of $\mathrm{EC}$ and discuss to what degree these findings can be conceptualised as a form of Pavlovian stimulus-stimulus learning. The formost accounts of EC are evaluated in the light of this discussion. It is argued that none of the present models of EC provide a satisfactory account of EC. In the second part of this chapter I discuss the development of a new model to account for the variety of EC effects. 


\section{The Associative Nature of Evaluative Conditioning}

In general, it is suggested that EC comprises the learning of an association between neutral stimulus and an affective stimulus. EC thus appears similar to Pavlovian stimulusstimulus learning. In Pavlowian conditioning $(\mathrm{PC})$, one leams an association between a neutral conditioned stimulus (CS; e.g., a tone, or a light) and a biologically relevant unconditioned stimulus (US; e.g., food, or an electric shock). Due to the formation of such an association, the mere presentation of a CS comes to elicit conditioned responding in anticipation of the US (e.g., appetitive behaviour when the US is food, or fear-motivated behaviour when the US is an electric shock) (Pearce \& Bouton, 2001). In EC, the neutral stimulus is usually referred to as the CS and the affective stimulus as the US. As De Houwer at al. (2001) note, there exist both similarities and discrepancies between findings in PC and EC.

Similarities between EC and PC. In PC an association between the CS and US gradually increases in strength to an asymptotic value with an increasing number of trials, which can be represented by a typical negatively accelerated learning curve. Some EC studies have found such a significant gradual change in affective value of the CS with an increasing number of trials (e.g., Baeyens, Eelen, Crombez, \& Van den Bergh, 1992). However, De Houwer et al. (2001) note that there have also been failures at demonstrating such a gradual acquisition of affect. This can be explained by arguing that the formation of an evaluative association occurs exceptionally rapid. Indeed, significant transfer of affect can be shown after just a single pairing of the CS and the affective US (Stuart, Shimp, \& Engle, 1987).

There are also several functional characteristics of $\mathrm{EC}$ that parallel $\mathrm{PC}$. One of these characteristics concerns the demonstration that $\mathrm{EC}$ is sensitive to a counterconditioning procedure. In demonstrating counterconditioning of acquired affective value, Bacyens et al. (1989) conducted a picture-picture EC study. After baseline ratings of the pictures, neutral CSs were paired with either positive, or negative USs. After the acquisition phase the CSs were rated as more positive (when paired with a liked picture) or more negative (when paired with a negative US), indicative of EC. After the post-acquisition assessment of likes and dislikes, some CSs were now paired with a US of opposite affective value as during the acquisition phase. The other CSs were either presented alone or not presented at all. After this phase of the experiment participants had to rerate all the CSs. The rating of the CSs that had been paired with the US of opposite affective value returned to baseline level, whereas evaluation of the other CSs did not change in comparison with the postacquisition ratings.

Hammer and Grabitz (1.996) demonstrated in two experiments evaluative sensory preconditioning, also using a picture-picture paradigm. In the preconditioning phase, participants received several sequential pairings of two neutral CSs. In the second phase, $\mathrm{CS}_{2}$ 
was pailred with a liked US. At test, transfer of positive affect was not only apparent for $\mathrm{CS}_{2}$ but also for $\mathrm{CS}_{1}$ that had not been paired with the US. This finding corresponds well with the demonstration of Pavlovian sensory preconditioning (see e.g., Rescorla \& Durlach, 1981 )

As in $\mathrm{PC}, \mathrm{EC}$ also appears sensitive to stimulus preexposure effects. For instance, Stuart et al. (1987) demonstrated that CS preexposure attenuates EC. More recently, De Houwer, Baeyens, Vansteenwegen, and Eelen (2000) also found some indication that preexposure to the CS may attenuate the transfer of affect to this CS in a subsequent EC procedure. Similarly, EC is sensitive to US preexposure. Hammerl, Bloch, and Silverthorn (1997) found that repeated preexposure of the US in a picture-picture study attenuated EC. This is in accordance with the finding that US preexposure leads to reduced conditioned responding in $\mathrm{PC}$ (see Randich \& Lolordo, 1979).

Another effect that parallels findings in $\mathrm{PC}$, is that $\mathrm{EC}$ is sensitive to US revaluation. Baeyens, Eelen, Van den Bergh, and Crombez (1992) conducted a picture-pichure study using photos of human faces. After an initial acquisition phase, the USs were revalued by accompanying the liked picture with negative adjectives and the disliked picture with positive adjectives. At test, the ratings of the CSs showed a shift in the similar direction as the shift in affective value of the USs due to revaluation. This effect was still present at a one-month follow-up.

Discrepancies between $E C$ and $P C$. Although $\mathrm{EC}$ appears to cllosely resemble $\mathrm{PC}$, there are some notable differences between $\mathrm{EC}$ and $\mathrm{PC}$. For example, contiguity between the CS and US is important in demonstrating PC. This is also certainly true for $\mathrm{EC}$, but as opposed to $\mathrm{PC}$, where the most learning is apparent when the CS directly precedes the US (i.e., a delayed conditioning procedure), EC works particularly well when the CS and the affective US are presented in compound (i.e., a simultaneous conditioning procedure) (see Rozin et al., 1998). Further, in contrast with general findings in PC, EC can be demonstrated using a backward conditioning procedure, where the US precedes the presentation of the CS (Martin \& Levey, 1978; Stuart et al., 1987). It should be noted though that this effect has not always been obtained (see Hammerl \& Grabitz, 1993).

A more notable discrepancy with $\mathrm{PC}$ is the finding that statistical contingency does not play an important role in the transfer of affect. Baeyens, Hermans, and Eelen (1993) manipulated the degree of statistical contingency between the CS and the US in a picturepicture study. Transfer of affect, indicative of EC, did not differ between a group in which the CS was always paired with the US, a group in which the CS was paired on half of the trials with the US, and a group in which the CS was paired with the US in only a third of the trials. Closely related to this discrepancy is the repeated finding that for $\mathrm{EC}$ to take place, one does not need to be aware of the contingency between the CS and the US. Although there is still considerable debate about the implicit nature of EC (see Field, 2000; 
Lovibond \& Shanks, 2002), the general conclusion is that EC does not require contingency awareness as opposed to PC (see De Houwer et al., 2001).

Another important discrepancy with $\mathrm{PC}$ is that $\mathrm{EC}$ appears to be highly resistant to an extinction procedure. Whereas in PC the learned response can be extinguished by presenting the CS without the US, such postacquisition nonreinforced exposure to the CS typically does not affect the acquired affective value of the CS (e.g., Baeyens, Crombez, Hendrickx, \& Eelen, 1995; De Houwer et al., 2000). This does not necessarily imply that $\mathrm{EC}$ is different from PC. Pavlovian conditioned responses too cannot be extinguished in the sense that they are unlearned. When a Pavlovian CS is presented outside the extinction treatment, conditioned responding typically recovers (see Bouton, 1993). It has been argued that such a renewal effect can also account for the apparent resistance of EC to extinction. Whereas the extinction of Pavlovian conditioned responding is measured during extinction treatment, the extinction of EC is usually assessed after an extinction procedure. The assessment of the affective value of the $\mathrm{CS}$ at test after extinction may be regarded as a novel situation, or context, allowing for the renewal of EC (Lipp, Oughton, \& Lelievre, 2003). Indeed, Lipp and colleagues (2003) demonstrated extinction of EC when assessing affective value of the CS during the extinction treatment. It should be noted though that the extinction of EC was less complete and far less rapid than the simultaneous extinction of Pavlovian conditioned skin conductance responding. Therefore, one can still argue that EC is exceptionally, though not completely, resistant to extinction.

Similar to the resistance to extinction, EC also appears to be resistant to modulation. In Pavlovian discrimination learning, responding to a CS can come under modulatory control of a feature stimulus when the CS is reinforced in the presence of, or when preceded by the feature stimulus (feature positive discrimination). Similarly, responding to the CS can come under modulatory control when it is reinforced only in the absence of the feature stimulus (feature negative discrimination). The feature stimulus is said to set the occasion for the CS to be reinforced (in feature positive discrimination training), or not reinforced (in the case of feature negative discrimination training) (Holland, 1983; Rescorla, 1985). Baeyens and colleagues (Baeyens, Crombez, De Houwer, \& Eelen, 1996; Baeyens, Hendrickx, Crombez, \& Hermans, 1998) have repeatedly failed to demonstrate such occasion setting in $\mathrm{EC}$.

Conclusions. In many respects EC appears similar to $\mathrm{PC}$. Nonetheless, considering the specific characteristics of $\mathrm{EC}$, particularly its resistance to extinction and the unimportance of contingency (awareness), EC is usually regarded as a form of associative stimulusstimulus learning which qualitatively differs from PC (but see Davey, 1994a). Since EC differs from PC, EC cannot be accounted for by well-defined models of associative learning, such as the Rescorla-Wagner model (Rescorla \& Wagner, 1972). Therefore, different models of $\mathrm{EC}$ have been proposed to account for both the similarities and discrepancies with Pavlovian learning. 


\section{Models of EC}

The conceptual categorisation model of EC. Davey (1994b) has argued that EC does not reflect associative learning at all, but rather conceptual categorisation. According to Davey, a CS usually has some features in common with the US it is paired with. Due to this paring, the common features become more salient and thus the CS is categorised as being more similar to the US. Field and Davey (1997) demonstrated how the pairing of stimulli promotes conceptual categorisation. In this study, participants were presented with pictures of faces of Martians and Venusians. The researchers constructed these faces and the exemplar Martian and Venusian face differed on six specific features. In the experiment, ambiguous alien faces (sharing as many Martian as Venusian features) were paired with more prototypical Martians or Venusians. At test, the ambiguous aliens were evaluated as more Venusian-like when paired with a prototypical Martian, whereas the ambiguous aliens that had been paired with the prototypical Venusian were rated as more Martianlike. Although these results seem to be at odds with the general finding in EC that the evaluative shift is in the direction of the US, Field and Davey state that these results nonetheless show how pairing of stimuli leads to categorisation and thus argue that such conceptuall categorisation may also play an important role in demonstrating EC.

Field and Davey (1999) point out that in several EC studies, CS-US pairs were constructed on the basis of perceptual similarity, hence promoting conceptual categorisation. Further, in a picture-picture study, they found no evidence for EC when the paired CSs and USs were perceived as dissimilar. Therefore, they argue that most demonstrations of EC are not the result of associative learning, but the result of an artefact due to the experimental paradign (see also Shanks \& Dickinson, 1990). De Houwer et al. (2001) however argue that in many EC studies, CSs were assigned to USs on a random basis, or stimuli were counterbalanced, thus controlling for perceptual similarity effects. Baeyens, De Houwer, Vansteenwegen, and Eelen (1998) further argwe that the conceptual categorisation account of EC cannot explain cross-modal EC. It is highly unlikely that the CS and the US are perceived as being similar in this situation. Furthermore, it cannot explain why US revaluation should specifically affect the evaluation of the CS that has been paired with the revalued US (see De Hotwer et all, 2001).

The holistic representation model of EC. Martin and Levey (1994) do not discard EC as an artefact, but like Davey (1.994b) they do not regard EC as the formation of an association. Martin and Levey describe $\mathrm{EC}$ as the automatic formation of a holistic representation containing elements of both the CS and the US. When a neutral and affective stimulus are presented in compound, they are automatically fused or integrated into a single representation. According to these authors, this fusion process constitutes one of the most 
primitive and basic forms of learning. In EC, the CS is thought to activate the holistic CSUS representation and thus the affective value of the US, accounting for the observed evaluative shift. It accounts for most findings in EC, but encounters some difficulty in explaining sensory preconditioning as demonstrated by Hammerl and Grabitz (1996). Since the formation of a holistic representation depends on the co-occurrence of the CS and the US, a CS that has never been paired with the US should not be able to acquire affective value according to the holistic representation account.

The referential learning model of $E C$. Baeyens and colleagues (Baeyens et al., 1992; Baeyens \& De Houwer, 1995) do describe EC in terms of the learning of an association, albeit different from Pavlovian learning. According to Baeyens et al. (1992), human associative learning comprises two distinct learning systems: a signal learning system and a referential learning system. The signal learning system controls PC. In PC one has to be aware of the contingency between the CS and the US to be able to adequately anticipate the US when the CS is presented. However, in EC, controlled by the referential learning system, the CS merely has to refer or activate the representation of the US for effective transfer of affect. The referential learning model thus requires far less information processing resources and hence does not require much conscious processing.

The referential learning model is very similar to the account of Martin and Levey (1994), but as opposed to their account of EC, Baeyens and De Houwer (1995) do not regard EC as the basis of more complex stimulus learning such as PC. The referential learning model also explains most results of EC studies. However, Stevenson, Boakes, and Wilson (2000) point out that this model only allows affective value to transfer. Stevenson and colleagues (Stevenson, Prescott, \& Boakes, 1995; Stevenson, Boakes, \& Prescott, 1998; Stevenson, Boakes, \& Wilson, 2000) have repeatedly demonstrated that not only affect can transfer, but other salient attributes of the US as well. For example, in one of their experiments, Stevenson et al. (2000) found that liking for a sucrose-paired odour was greater than liking for a citric-paired odour. Next to the transfer of affect, the sucrose-paired odour was also rated as more sweet and the citric-paired odour as more sour at test. De Houwer et al. (2001) acknowledge that the referential learning model cannot account for this result, unless it is presumed that the referential learning system controls all but Pavlovian anticipatory responding.

In summary, the present models of EC cannot account for all demonstrations of EC and are difficult to distinguish as they render roughly the same set of predictions. De Houwer et al. $(2001$, p. 866$)$ conclude that: “... these models tell us little about the specific processes that underlie $\mathrm{EC}$ and thus do not permit the formulation of precise hypotheses. It is therefore imperative that existing theories are reexamined and refined or that new models are proposed." 


\section{Stimulus Generalisation and Configural Learning as a Model of EC}

In the first part of this chapter, $I$ briefly reviewed and summarised the different findings concerning $\mathrm{EC}$ and discussed to what degree $\mathrm{EC}$ can be conceptualised as a form of Pavlovian stimulus-stimulus learning (for a much more comprehensive review concerning this topic I refer to De Houwer et al, 2001). The present models of EC as described above all

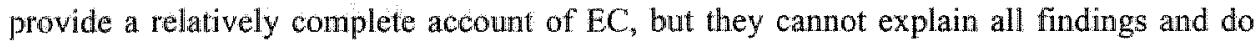
not generate very precise predictions. Typically, these modells either fail to account for the apparent importance of perceptual similarity between stimuli in demonstrating $\mathrm{EC}$, or fail to explain $\mathrm{EC}$ when paired stimuli are not perceptually similar. In reviewing the findings in EC studies I argue in the following sections that EC is perhaps best understood in terms of stimulus generalisation and configural learning.

In 1987, Pearce devised at model of stimulus generalisation, describing the role of generalisation and configural learning in the context of PC. Pearce presumes in accordance with Atkinson and Estes (1963) that one possesses a buffer containing representations of all stimuli present in the context to which one is exposed. According to Pearce, when a stimulus is presented it activates a representation of that particular stimulus in the buffer. Through a process of spreading activation it can also activate representations of stimuli that are not presented but share features in common with the presented stimulus. So when a CS X has acquired excitatory associative strength due to pairings with a US (e.g., food), some conditioned responding will be elicited by a novel CS $Y$ when this stimulus shares features in common with X. I argue that this principle of stimulus generalisation as formulated by Pearce can allso be applied to EC. According to the present model then, the hedonic shift observed in EC is due to generalisation of affective value from the affective US to the CS. Similar to the generalisation of associative strength, 1 argue that the amount of generalised affective value is determined by the perceptuall similarity of the neutral stimulus with the affective US. Suppose that a stimulus $X$ has a certain affective value. The degree of generalised affect from $X$ to a stimulus $Y$ then depends on the perceptual similarity of $X$ and $Y$ and, of course, the affective value of stimulus $X$. Equation 1 represents this relationship and it directly corresponds with the equation of generalised associative strength described by Pearce (1987).

$$
a_{y}={ }_{x} S_{r} \times A_{x}
$$

Parameter $a_{Y}$ represents the total amount of generalised affective value to stimulus $Y$. The value of $a_{Y}$ depends on the similarity between stimuli $X$ and $Y$, represented by the parameter $X S_{Y}$, which can also be described as the degree to which stimulus $Y$ is able to 
directly activate a representation of $\mathrm{X}$. Affective value of stimulus $\mathrm{X}$ is represented by the parameter $A_{\mathrm{X}}$ and can take on a value anywhere between -1 (negative valence) and 1 (positive valence).

The $S$ parameter in Equation 1 requires some more specification. In accordance with Pearce $(1987,2002)$ I argue that the similarity $(\$)$ between two stimuli depends on the number of common features and the total number of activated representations by each stimulus. Pearce $(1987,1994)$ formulates similarity as displayed in Equation 2.

$$
{ }_{x} S_{Y}=\frac{n_{C}}{n_{X}} \times \frac{n_{C}}{n_{Y}}
$$

Equation 2 describes how $S$ depends on the ratio of the number of common features $n_{\mathrm{C}}$ (not only including the perceptual features of the stimuli, but also the context in which these stimuli are presented) and the total number of activated representations by stimullus $X$, as well as the ratio of the number of common features and the total number of activated representations by stimulus $\mathrm{Y}$. If one assumes that stimulus $\mathrm{X}$ and $\mathrm{Y}$ are equally intense and thus activate the same number of common representations, Equation 2 can be rewritten as Equation 3.

$$
x S_{Y}=\frac{n_{C}{ }^{2}}{\left(n_{X} \times n_{Y}\right)}
$$

Although formulated somewhat differently than Davey (1994b), I also argue that the perceptual similarity between stimuli plays a key role in demonstrating EC. Using a picture-picture paradigm, Field and Davey (1999) demonstrated the importance of perceptuall similarity in EC. Pictures of human faces were evaluated after which neutral pictures (CSs) were paired with either liked or disliked pictures (the USs). At test, participants had to rerate the pictures. Shifts in affective value were found, but only for the CSs that were categorised as being similar to the US they had been paired with. Moreover, such transfer of affect was also found in a control group that had received explicitly unpaired presentations of the CSs and the USs, and in another control group of participants who simply had to rate the pictures twice.

Although perceptual similarity certainly plays an important role in EC, De Howwer et al. (2001) emphasise that perceptual similarity is not a prerequisite in demonstrating EC. The stimulus generalisation model of EC as presented thus far, also cannot provide an account of EC when the CS and the US are perceptually dissimilar. However, I argue that the generalisation of affective value can be promoted through the formation of a configural representation of the CS and the affective US. The potentially important role of configural representations in human associative learning has already been noted by several research- 
ers (e.g, Shanks, Charles, Darby, \& Azmi, 1998; Shanks, Darby, \& Charles, 1998; Stevenson et all., 2000).

According to Pearce (1987), a configural representation is formed when two or more stimuli are presented in compound. Pearce (2002) has described how such configural representations may play a role in animal flavour-flavour learning, a phenomenon that bears elose resernblance to the EC paradigm. In animal flavour-flavour learning, animals receive pairings of artificial flavours paired with an inherently preferred flavour (e.g., a sweet flavour). This leads to the acquisition of a conditioned flavor preference for the previously neutral flavour. As in EC, conditioned flavour preferences are acquired very rapidly and are highly resistant to an extinction procedure (see Capaldi, 1996). Pearce states that whenever a neutral flavour is paired with the preferred flavour, the co-activation of the representation of the neutral and preferred flavour leads to the formation of a configural representation, comprising elements of both flavours. When the neutral flavour is presented at test, a representation of the preferred flavour is activated indirectly through the activation of the configural representation. The degree to which the initially neutral flavour is able to activate the configural representation rapidly increases, requiring but a few trials.

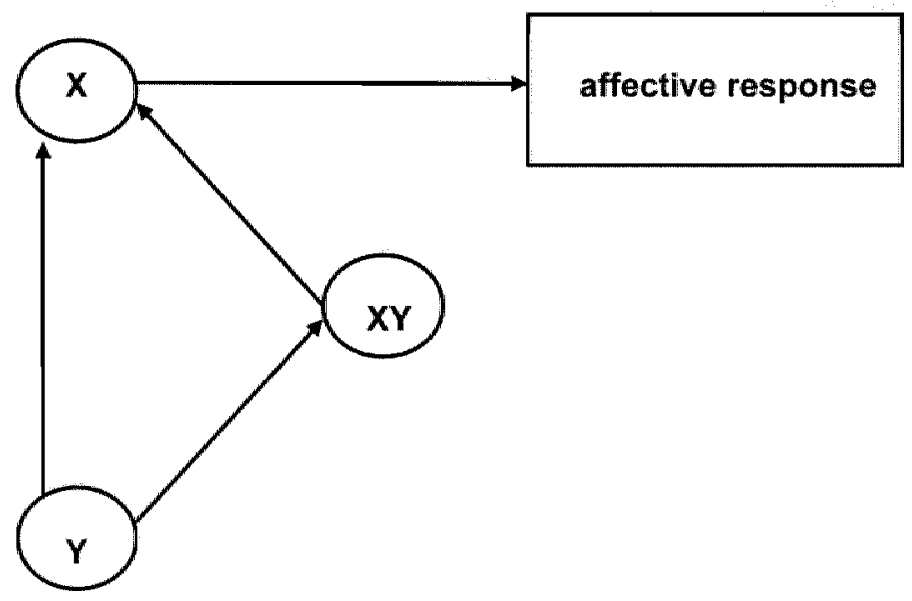

Figure 9.1. A schematic representation of how an initially neutral stimulus $Y$ acquires affective value from an affective stimulus $X$. Due to pairing of $X$ and $Y$, a configural $X Y$ representation is formed. When $Y$ is presented, affective value of $X$ will generalise to $Y$ to the extent that $Y$ is capable of directly and indirectly activating a representation of $X$.

Figure 9.1 displays how this notion of configural learning can also be applied to $\mathrm{EC}$. As described by the figure, when an affective stimulus $X$ is paired with a neutral stimullus $\mathrm{Y}$, the co-activation of the representation of $\mathrm{X}$ and $\mathrm{Y}$ leads to the formation of an 
$X Y$ configural representation. When $Y$ is presented at test, it activates the representation of $\mathrm{X}$ directly to the extent $\mathrm{X} S_{\mathrm{Y}}$, and indirectly through the activation of XY to the extent $\mathrm{Y} S_{\mathrm{XY}}$ " $\mathrm{x} \mathrm{S}_{\mathrm{X}}$. As such, the stimulus generalisation model does not preclude the possibility of cross-modal EC, in contrast to Davey"s model (1994b). However, it does predict that crossmodal $\mathrm{EC}$ is more difficult to demonstrate. Further, it predicts that effective $\mathrm{EC}$ requires but a few CS-US pairings.

Summarised, affective value can generalise both directly and indirectly from multiple affective sources. Therefore, Equation 1 can be extended to the more general Equation 4 , which again directly corresponds with Pearce's 1987 notion of generalisation of associative strength in the context of PC. Equation 4 provides an equation for determining the degree of generalised affective value to stimulus $\mathrm{Y}$ from $n$ stimuli similar to $\mathrm{Y}$ having sonme affective value (positive, or negative).

$$
a_{Y}=\sum_{n} s_{Y} \times A_{n}
$$

The present stimulus generalisation model of EC explains the irrelevance of contingency in EC by stating that EC partly depends on the formation of a configural representation. This latter process of configuring is thought to occur automatically relying solely on the co-activation of the representations of the presented stimuli. Pearce's 1.994 comectionist model of configural learning describes the formation of a configural representation as an automatic and immediate non-iterative process, as opposed to the gradual formation of a Pavlovian association between stimuli. Differing levels of contingency should thus exert no effect on the demonstration of $\mathrm{EC}$ and one need not be aware of the specific contingency between the CS and the US.

The model also predicts that simultaneous presentations off the CS and the US should be most effective in demonstrating EC. Although Rozin et al. (1998) note that this seems to be the case, EC has been frequently demonstrated using forward pairings of the $\mathrm{CS}$ and the US. In its present form the stimulus generalisation model of EC does not permit the formation of a configural representation when the CS and the US are presented sequentially. As Pearce (2002) notes though, the model can accommodate the formation of a configural representation when stimuli are presented in a serial compound, if it is assumed that activation of the representation of a stimulus is not terminated instantly with the offset of the presented stimulus, but slowly decays.

In summary, the present model predicts that perceptual similarity plays a key role in demonstrating EC. It also predicts that contiguity, but not contingency, is important in demonstrating EC. Apart form these initial predictions, the stimulus generalisation and configural learning model of $\mathrm{EC}$ can account for most typical EC findings.

Application to extincrion. As the model describes EC in terms of stimulus generalisation and configural learning, the model predicts that the acquired affective value should 
be extremely resistant to extinction. Once a configural representation is formed, nonreinforced exposure to the CS should not affect its ability to activate a representation of the configural CS-US representation, and hence generalisation of affective value should be relatively unaffected by an extinction procedure.

Application to modulation. The model predicts that EC should resist modulation as Baeyens and colleagues (Baeyens et al., 1996, Baeyens et al., 1998) demonstrated. In one of their experiments (see Baeyens et al., 1996) the participants tasted a flavor (A) having a certain colour $(X)$ paired with Tween (a taste which was generally rated as highly aversive by the participants). When the flavour was presented without the specific colour, the flavour was not paired with Tween. Colour did not come to modulate evaluative responding at all. At test, evaluative shifts were observed for the flavour whether or not presented in compound with the colour. The model explains this pattern of findings by suggesting that during discrimination training an $\mathrm{XA}^{-} \mathrm{US}_{\text {Tween }}$ configural representation was formed. Due to $X$ being of a different sensory modality than both $A$ and the US, the similarity between $X$ and $X A-U S_{\text {Tween }}$ would be much smaller than the similarity between $A$ and $X A-U S_{\text {Tween. }}$. Generalisation of affective value from the US would thus be limited primarily to stimulus A and the XA compound as was demonstrated by Baeyens and colleagues (1996).

Application to counterconditioning. Baeyens and colleagues (1989) found that EC is sensitive to a counterconditioning procedure. Although at face value this finding appears to imply that EC reflects some form of associative learning, the present nonassociative model of EC can accommodate this pattern of results. According to the model, in the initial acquisition phase a configural representation is formed containing both elements of the CS and the affective US 1 . When in the following phase the CS is now paired with another affective stimulus $\left(\mathrm{US}_{2}\right.$ ) that has an affective value directly opposite to $\mathrm{US}_{1}$, presentation of the CS at test will activate a representation of both USs. Assuming that the CS activates the USs to a similar extent, the net generalised affective value to the CS will approach zero.

Application to US revaluation. Baeyens et al. (1992) showed that EC is sensitive to US revaluation. The present model can accommodate the observed US revaluation effect without referring to the formation of an association. The model predicts that when an affective US is revalued, the CS that has previously been paired with this US will show an evaluative shift in the same direction as the US, because at test it activates the representation of the revalued US through the activation of the previously acquired configural CS-US representation.

Application to sensory preconditioming. As described above, the finding of evaluative sensory preconditioning (-Tammerl \& Grabitz, 1996) posed serious implications for the holistic representation account of EC proposed by Martin and Levey (1994). Although the present model of $\mathrm{EC}$ is very similar to the notion of the formation of a holistic representation put forth by Martin and Levey, it does not preclude sensory preconditioning. In the furst phase of the paradigm, it is predicted that a configural $\mathrm{CS}_{1}-\mathrm{CS}_{2}$ representation is 
formed. In the subsequent conditioning phase, a $\mathrm{CS}_{2}-\mathrm{US}$ configural representation will be formed. When $\mathrm{CS}_{1}$ is presented at test, it will be able to activate the representation of the US directly through its perceptual similarity with the US and indirectly through the activation of the configural representations.

Application to stimulus preexposure. Another effect in EC that appears to demonstrate the associative nature of $\mathrm{EC}$ is the detrimental effect of stimulus preexposure on $\mathrm{EC}$ (e.g., Stuart et al., 1987; Hammerl et al., 1997). The present model cannot explain the detrimental effects of CS (or US) preexposure on EC, unless it is assumed that such preexposure leads to perceptual habituation to the preexposed stimulus, hence limiting the formation of a configural representation during the subsequent conditioning phase. Hammerl et al. have made a somewhat similar proposal in explaining the effects of US exposure in $\mathrm{EC}$. In concordance with Randich and Lolordo (1979), Hammerl and colleagues state that repeated US exposure leads to habituation to the US and as a consequence the US will loose some of its affective value. As such, both preexposure and postexposure of the US should attenuate $\mathrm{EC}$.

Although the present model of EC encounters some difficulty in explaining stimulus preexposure effects on $\mathrm{EC}$, it should be noted that these effects have not been studied extensively as of yet. Furthermore, the detrimental effect of CS preexposure on EC has not always been found to be a reliable effect (see De Houwer et al., 2000; Stevenson et al., 2000).

\section{Tests of the Model}

As described above, the stimulus generalisation model of EC accommodates most EC findings. However, the question arises then how to test the present model.

One of the primary assumptions of the present stimulus generalisation model is that similarity between stimuli is an important factor in demonstrating EC. In EC a CS will show an evaluative shift in the direction of the affective value of the activalted representation of the affective US it has been paired with. If a CS is thus paired with both a positive and negative stimulus, the evaluative shift at test will be determined by the degree in which the representations of these different USs are activated. When both USs are activated to the same extent and have directly opposing affective values, no EC will be apparent at test.

However, consider the following discrimination. A neutral stimulus $A$ is paired with a negative US, another stimulus $B$ is paired with a positive US. Next to these paired presentations, $A$ is also presented in compound with another CS C and paired with the positive US, and $B$ is also presented in compound with $C$ and paired with a megative US. This then leads to an $\mathrm{A}-\mathrm{US}_{1}{ }^{-} / \mathrm{B}-\mathrm{US}_{2}{ }^{+} / \mathrm{AC}-\mathrm{US}_{2}{ }^{+} / \mathrm{BC}-U \mathrm{~S}_{1}{ }^{-}$discrimination. How would such a discrimination affect the transfer of affect to $A, B$, and $C$ separately? 
The referential model states that an evaluative association depends solely on the cooccurrence of the CS and the US. As each CS in this case co-occurs with both a positive and negative US, no significant $\mathrm{EC}$ is expected to be observed according to this model. The conceptual categorisation model renders the same prediction when one assumes that all individual stimuli are equally similar to one another and the positive and negative USs have directly opposing affective values. The holistic representation model does not specify when or how a particular holistic representation will be retrieved and thus does not render a specifie prediction in this case: In contrast to these existing models of EC, the stimulus generalisation model predicts that one can 'solve' the discrimination. It predicts that $\mathrm{A}$ shows a negative shift, B a positive shift and no substantial evaluative shift for stimulus C. Presentation of $\mathrm{A}$ is more likely to lead to the activation of the representation of the negative US, as $\mathrm{A}$ is more similar to the configural $\mathrm{A}-\mathrm{US}_{\mathrm{l}}$ stimulus than the configural $\mathrm{AC}-$ $\mathrm{US}_{2}{ }^{+}$representation. In contrast, $\mathrm{B}$ will more strongly activate a representation of the positive US, as stimulus $\mathrm{B}$ is more similar to the configural $\mathrm{B}-\mathrm{US}_{2}{ }^{+}$stimulus than the $\mathrm{BC}-$ $\mathrm{US}_{1}$ " representation. Presentation of $\mathrm{C}$ will activate the representation of both the positive and negative US to a similar extent, thus limiting EC. Whether one truly can 'solve' such a discrimination remains to be investigated.

Another prediction of the present model concerns compound conditioning. In $\mathrm{PC}$, when two CSs are presented in compound and paired with a US, this compound stimulus will be just as effective at eliciting conditioned responding as any other single CS paired with this US. The present model, however, predicts that when a compound comprising two neutral CSs (e.g., A and B) is paired with an affective US $Y$, the transfer of affective value to this compound will be less than the transfer of affective value from $Y$ to a CS comprising a single neutral stimulus $X$. When the $A B$ compound is presented at test, this will activate the $A B-Y$ configural representation that in turn activates the $A, B$ and $Y$ representations. As the similarity between the $A B-Y$ representation and $Y$ is smaller than the similarity between the $X-Y$ representation and $Y, Y$ will be less activated by presentation of the $A B$ compound as compared to $X$ and thus, the transfer of affective value from $Y$ to the $A B$ compound will be less than the transfer from $Y$ to $X$. Additionally, the $A B$ compound also activates representations of its neutral elements $A$ and $B$ separately, and this should further limit the transfer of affective value. None of the other models of EC specifically makes such a prediction, but again, whether compound conditioning is indeed different in EC in comparison with $\mathrm{PC}$ remains to be investigated. 


\section{Concluding Comments}

The primary aim of the present chapter has been the development of a niew model of $\mathrm{EC}$. The model accommodates most $\mathrm{EC}$ findings and generates specific hypotheses concerning $\mathrm{EC}$ effects. It can be argued that previous models of $\mathrm{EC}$ either fail to explain the importance of perceptual similarity in demonstrating EC (e.g., Baeyens et al., 1992; Martin \& Levey, 1994), or fail to explain the possibility of the transfer of affect when stimuli are perceptually dissimilar (Davey, 1994b). By describing EC in terms of generalisation of affective value and emphasising the role of configural learning in such generalisation, the importance of perceptual similarity is incorporated in the present model while not precluding the possibility of $\mathrm{EC}$ when stimuli are perceptually dissimilar. Therefore, it can be argued that the present model provides a more comprehensive account of EC.

As noted above, the present stimulus generalisation model of $\mathrm{EC}$ is an adaptation of the 1987 Pearce model of stimulus generalisation in the context of Pavlovian learning. Since this model can be so easily modified to account for both the generalisation of associative strength and affective value, there is no reason to assume that stimulus generalisation should be restricted to the generalisation of these two potential stimulus attributes. So although the generalisation model is presented here as a model of EC, it can also explain the simultaneous transfer of other salient stimulus attributes.

Although the stimulus generalisation and configural learning account of $\mathrm{EC}$ provides a more complete and specific account of $\mathrm{EC}$, as any other model it requires rigorous testing. I agree with De Houwer et al. (2001) that future EC studies should be explicitly aimed at testing the boundary conditions of EC. Such studies should provide results with which one can evaluate the merits of the present model. 
EXCESSTV APPETTE 


\section{GENERAL DISCUSSION}

No matter what kind of diet you are on, you can usually eat as much as you want of anything you don't like.

Walter Slezak 
The central theme of the present thesis concerned the Pavlovian nature of an excessive appetite, such as an intense craving for drugs or alcohol, or a strong preference for highcaloric tastes. It can be concluded that conditioning plays an important role in the acquisition of an excessive appetite. However, at the same time it is clear that not all drug che reactivity and not all forms of acquired food likes can be ascribed to Pavlovian learning processes. The following sections provide an overview of the results described in detail in the preceding chapters and a discussion of theoretical and clinical implications of the present findings.

\section{Overview}

In chapter 1, it was tested whether blocking of learning a causal relation between a potential cause (e.g., a chemical substance) and a specific outcome (e.g., symptoms of a disease) is affected by the magnitude of the outcome. If so, this would provide support for the notion that human causal inference is governed by Pavlovian conditioning processes. Indeed, such an influence of outcome magnitude was demonstrated, but this did not necessarily depend on trial-by-trial learning.

In chapter 2 , it was hypothesised that both cue-elicited urges and psychophysiological arousal in drug dependent patients are conditioned drug responses that can be extinguished when one is repeatedly exposed to substance cues, but prevented from substance use. Such cue exposure, though, was not effective in extinguishing psychophysiological cue reactivity. Although cue-elicited drug urges did extinguish, this extinction was not limited to the patients receiving cue exposure with response prevention.

The question whether cue-elicited urge to smoke directly interferes with cognitive performance, as hypothesised by Tiffany (1990), was investigated in chapter 3. No strong evidence for such a relation was found. Smokers demonstrated slower reaction times on a memory search task when being nicotine deprived, and this psychopharmacological effect was merely partially mediated by the urge to smoke.

In chapter 4 , it is argued that the inefficacy of cue exposure treatment in preventing relapse of addictive behaviour can be attributed to renewal of previously extinguished cue reactivity when the patient is exposed to cues outside the treatment setting. Such renewal of extinguished conditioned responding has been demonstrated in animals, but does it also occur in human subjects? This was investigated in chapter 5 . It was found that humans too demonstrate renewal of conditioned responding, but the results also appeared to indicate that this effect was controlled by higher-order cognitive processes, rather than associative learning. 
In chapter 6 , it was investigated how and to what degree environmental context af fects the activation of aloohol-related cognitions in social drinkers. The results of primary interest indicated that an alcohol-related environment direetly enhances accessibility of the alcohol memory network in social drinkers, similar to the effect of a semantic aloohol prime (i.e., the word "beer").

Chapter 7 concemed the hypothesis that sensory-specific satiety is less pronounced in obese women. It has been suggested that less sensory-specific satiety may promote overeating. No evidence was found for this hypothesis though. On the contrary, obese women tended to demonstrate stronger sensory-specific satiety than normal-weight women.

The question whether macronutrients, that is carbohydrates and dietary fat, have differential reinforcing potencies in the acquisition of a conditioned taste preference in humans, was investigated in chapter 8 . Results indicated that indeed there was such a difference; carbohydrate-paired tastes increased in palatability as opposed to isocaloric tastes paired with a high dietary fat content.

Taste preferences can also be acquired when a neutral taste is paired with an already liked taste (e.g., the sweet taste of sugar). Such transfer of affective value has been termed evaluative conditioning. However, in chapter 9 , it is argued that the transfer of affective value to a neutral stimulus when paired with an affective stimulus (positive, or negative) does not reflect associative learning (i.e., conditioning), but stimulus generalisation.

\section{Learning and Drug Consumption}

Learning is thought to play an important role in the etiology of addictive behaviour. Associative models of addiction state that due to experience with drug consumption, drug related cues come to function as conditioned drug stimuli associated with the unconditioned stimulus drug intake. As such, these cues come to elicit cue reactivity comprising psychophysiological reactions that are subjectively experienced as craving that subsequently motivates drug self administration. In support of such a view, cue reactivity has indeed been found and the dlegree of such reactivity correlates with the amount of drug taking experience (see Drummond, Tiffany, Glautier \& Remington, 1995). However, typical psychophysiological reactions to drug cues appear to resemble a more general arousal response and do not correlate very highly with cue-elicited craving and thus some researchers have argued that this reactivity does not necessarily reflect conditioned drug responding (e.g. Carter \& Tiffany, 1999). In chapter 2, the hypothesis that psychophysiological cue reactivity can be extinguished was investigated in drug and alcohol dependent patients. No sup- 
port for this hypothesis was found though; that is, cue-elicited psychophysiological reactivity did not extinguish, whereas cue-elicited drug urges did. One reason for this pattern of findings is that the psychophysiological measures in this study were primarily measures of general arousal responding rather than more drug-specific psychophysiological indices. It may be possible that other cue-elicited physiological responses do correlate with cueelicited urges and can be extinguished. As argued in chapter 2, although it may be relatively easy to control one's urges when exposed to cues, this does not mean that one should become indifferent to such exposure. Therefore, one still demonstrates arousal responding when being exposed to cues, even when these cues no longer elicit strong craving.

Carter and Tiffany (1999) have argued that cue-elicited arousal reflects frustration of being exposed to cues but prevented from drug use. Such arousal responding is in line with Tiffany's 1990 cognitive model of drug urges and drug use. According to Tiffany's cognitive model of addiction, exposure to cues activates a drug action schema. When execution of this schema is impeded, automatic drug use is impossible and hence nonautomatic cognitive resources are addressed to ensure drug use. The activation of nonautomatic cognitive processes reflects mental stress, which then is expressed as urges and psychophysiological arousal. This model thus explicitly states that Pavlovian conditioning does not underlie cue reactivity. The primary prediction of this model is that cue exposure should lead to urges and as these urges reflect the operation of capacity-limited nonautomatic cognitive processes the urge to use a given drug should interfere with performance on tasks requiring the same cognitive resources. The hypothesis that urges directly interfere with cognitive performance was tested in chapter 3. No strong support was found for this hypothesis. When smokers experience a strong urge to smoke, this does not necessarily attenuate cognitive performance, such as the performance of a choice RT task as described in chapter 3 . The notion, then, that urges or craving are the result of the activation of nonautomatic cognitive processes rather than Pavlovian drug conditioning does not appear to be tenable. Further, Tiffany's model of drug urges does not provide an explanation for the apparent extinction of urges or craving when applying cue exposure with response prevention (CERP) and the absence of a correlation between cue-induced psychophysiological arousal and craving (Drummond, et al., 1995; see also chapter 2).

The efficacy of CERP in the treatment of addiction has been investigated in previous studies and it has been demonstrated to effectively extinguish cue-elicited urge responding. Cue-elicited urges are thought to be one of the primary motivations for prolonged drug use and thus the successful extinction of such urge responding should limit the probability of a relapse. As described in chapter 4, CERP, however, does not affect the probability of a relapse at all. In chapter 4 it is argued that this is due to renewal. Renewal refers to the return of an extinguished conditioned response when exposed to the conditioned stimulus outside the extinction treatment context. In other words, extinction does not entail the unlearning or breaking of the link between conditioned stimulus and uncon- 
ditioned stimulus. Such renewal of Pavlovian conditioned responding has been frequently demonstrated in animals and researchers have suggested that this implies that extinguished cue-elicited urges can return outside the treatment setting, thus explaining the limited effect of CERP on the probability of relapse (see e.g., Conklin \& Tiffany, 2002; chapter 4). However, whether humans too demonstrate renewal of extinguished conditioned responding had not been investigated before. This then was tested in chapter 5 in a series of three experiments. It was found that humans, like laboratory animals, do demonstrate renewal of extinguished conditioned performance. However, not all results replicated the animal findings on the renewall effect, suggesting that the extinction of conditioned responding in humans is controlled by more higher-order cognitive processes.

The renewal effect underscores the relevance of contextual variables in the etiology of addictive behaviour. Not only does context play an important role in extinguishing reactivity (chapters 4 and 5), it also plays a rolle in displaying cue reactivity. Indeed, about a third of drug dependent patients typically do not demonstrate any craving when exposed to cues in a treatment context (Dawe \& Powell, 1995). Drummond (2000) has thus suggested that environmental context can control reactivity to more punctate drug-related cues. When drug cues are presented in a drug-related environment, these cues predict drug intake and hence elicit cue reactivity, but when they are presented in an enviromment that is not related to drug intake at all, the same cues may elicit little if any cue reactivity (see also Wertz \& Sayette, 2001). Similarly, Wall and colleagues $(2000 ; 2001)$ have found that the activation of alcohol-related cognitions is more pronounced in a bar than in a laboratory setting. As these drug-related concepts stored in memory can be activated by exposure to cues, such activation of drug-related cognitions can also be regarded as a form of cue reactivity (see also Glautier \& Spencer, 1999; Schulze \& Jones, 2000). To determine how context affects the activation of alcohol-related cognitions stored in memory was tested in chapter 6 . It was found that priming alcohol-related cognitions using a semantic verbal cue was only effective in an alcohol-neutral context. However, overall participants demonstrated stronger activation of alcohol-related cognitions in a pub-like environment. This then implies that an alcohol-related context does not hierarchically control responding to cues, but functions as a drug cue, itself capable of directly elliciting cue reactivity.

It is clear from the present results that cue-elicited craving can still be conceptualised as a Pawlovian conditioned drug response. However, it should be noted that the existing conditioning models of addictive behaviour cannot account for the apparent cueelicited physiological arousal responding. One exception may be the incentive sensitisation model of Robinson and Berridge (1993; 2000). Robinson and Berridge argue that drug consumption leads to progressive neural sensitisation of the reward system in the brain (i.e, the ventral tegmental area), which in return leads to the gradual development of a compulsive wanting of the drug, a process that Robinson and Berridge have termed incentive sensitisation. In rats, such sensitisation is expressed as increased psychomotor behav- 
iour (or behavioural arousal responding). This increase in psychomotor behaviour as a result of incentive sensitisation, however, is only expressed by the rat when placed in the enviromment in which it received previous administrations of a particular drug. In other words, arousal responding as the result of previous drug administration is under the control of drug-related cues, that is, the drug administration environment. This model then can accommodate the pattern of cue-elicited arousal in drug dependent patients. However, as Robinson and Berridge ascribe such cue controlled expression of incentive sensitisation to Pavlovian conditioning, their model cannot explain why cue-elicited psychophysiological arousal is apparently resistant to extinction.

\section{Learning and Eating}

The simplest form of learning from experience is learning about the relevance of a single event. An example of such learning is habituation. Habituation can be defined as the decrease in the strength of a response to a stimulus due to repeated exposure to that stimulus (Lieberman, 1993). Habituation to the sensory characteristics of a food is the primary explanation for sensory-specific satiety; the decrease in palatability of a food when eaten as compared to other foods uneaten. The function of sensory-specific satiety appears to be twofold: (i) promoting meal termination and (ii) promoting the motivation to eat a more varied meal. It has been suggested that obese persons may demonstrate less sensoryspecific satiety (SSS), hence promoting overeating of a single meal (Raynor \& Epstein, 2001 ). This hypothesis was tested in chapter 7 , comparing SSS between obese and normalweight women. It was found that the obese women displayed stronger SSS than the normal-weight women. The normal-weight women did display a significant decrease in the liking of a test drink, but displayed a similar decrease in palatability of a control drink, whereas the obese women specifically displayed at strong decrease of the palatability of the test drink as compared to the palatability of a control drink. It can thus be concluded that the defining individual differences in SSS between obese and nomal-weight women is that a decrease in palatability of a food eaten generalises easily to other tastes in normal-weight women, but not in obese women. This then implies that dietary variety attenuates SSS particularly in obese persons. This notion is corroborated by the finding by McCrory and collleagues (1.999) who demonstrated that the degree of obesity is related to the degree of dietary variety of specifically high-caloric food items.

Although SSS explains why obese persons tend to eat a more varied diet, it does not explain their apparent fat preference. Habituation then falls short in explaining the acquisition of such a taste preference, but Pavlovian conditioning can explain the development of such preferences. Conditioned taste preferences are thought to be the results of a 
learned association between the conditioned stimulus taste and the unconditioned stimulus energy density of the taste. Nutrients provide such energy, and as fat delivers the most energy per gram of all mutrients (i.e., $9 \mathrm{kcal} / \mathrm{g}$ ), a preference for taste of food with a high fat content should be conditioned most easily. Indeed, energy density has been found to be an important determinant of conditioning taste preferences. However, Sclafani (1997) has argued that nutrients each have their own specific postingestive effects apart from delivering energy and thus predicts that nutrients may have different reinforcing properties when caloric density is controlled for. Indeed, Lucas and Sclafani (1999a) demonstrated in rat subjects that a preference for a taste paired with carbohydrates was acquired more rapidly than a preference for a taste paired with an isocaloric fat content. Whether such an effect also occurs in humans was tested in chapter 8. It was found that the taste of a yoghurt drink paired with a carbohydrate content increased in both palatability and preference as compared to a low caloric control taste and an isocaloric yoghurt paired with fat. These results replicate the findings by Lucas and Sclafani, and it is argued that perhaps the differential satiating effects of nutrients account for the results. However, such an explanation warrants further research as this pattern of results can also be explained in terms of flavour-flavour evaluative conditioning.

Flavour preferences can also be acquired by pairing a taste with an already preferred taste. Whereas energy density and the postingestive effects of nutrients are clearly rewarding biologically relevant stimuli, thus capable of reinforcing neutral tastes paired with these stimuli, already liked tastes do not necessarily provide such biologically relevant postingestive effects (e.g., saccharine). Nonetheless, the sweet taste of noncaloric saccharine has been found to be very effective in conditioning a food preference (Fedonchak, 1997). In chapter 9 , it is argued that such evaluative conditioning does not necessarily reflect Pavlovian conditioning. Evaluative conditioning, the acquisition of likes and dislikes, or preferences and aversions by pairing a neutral stimulus with an affective stimulus (positive or negative) is perhaps best described in terms of stimulus generalisation and configural leaming. If one defines Pavlovian conditioning as the learning of a predictive or causal relation between an antecedent event and a specific outcome, then one nust conclude that evaluative conditioning is not some special form of Pavlovian conditioning at all.

In general, the studies described in chapter 7 through 9 support the notion that changes in food preferences can be the result of learning, although not necessarily the result of Pavlovian conditioning processes. Understanding how learning processes affect changes in taste preferences might provide insights into how learning contributes to obesity. Obese persons typically demonstrate an acquired fat preference (Rissanen, at al., 2002). Fat preferences have been found more difficult to condition and thus conditioning at first sight does not appear to be a very likely candidate explaining the fat preference typically observed in obese persons. However, it is a fallacy to state that obese (as compared to 
nomal-weight) persons exclusively show a fat preference, such as for example a taste preference for pure olive oil. Indeed, obese persons do excessively prefer high-fat food items, but these items are usually also high in either carbohydrate or protein content. Interestingly, obese men and women tend to differ in their preference for carbohydrate-rich and protein-rich foods with the obese men preferring foods with a combined high protein and fat content (e.g, meat) and the obese women preferring foods with a combined high carbohydrate and fat content (e.g., chocolate cake) (see Drewnowski, Kurth, Holden-Wiltse, \& Saari, 1992). In laboratory animals; both carbohydrates and proteins have been found to lead to the rapid acquisition of a taste preference (Fedorchak, 1997). Fat content appears to lead to a slower acquisition of a conditioned taste preference that, however, once acquired is much stronger and pervasive than carbohydrate conditioned taste preference (Lucas \& Sclafani, 19996). It is thus possible that a fat preference is learned through the development of a taste preference for carbohydrate- or protein-rich foods.

\section{Clinical Implications}

As stated in the general introduction to this thesis, the main relevance of studying the learned nature of an excessive appetite is the potential implications for clinical practice. As of yet, both obesity and addiction have been found to be extremely resistant to treatment (Stunkard, \& Penick, 1979; McLellan, O'Brien, Lewis, \& Kleber, 2000). In the treatment of addiction, kicking the habit is not much of a problem, but remaining drug abstinent after treatment is. Similarly, losing weight is not the problem for obese persons, but maintaining weight loss is.

In the treatment of addiction it has been found that craving plays an important role in the maintenance of addictive behaviour (see e.g., Swan, Ward, \& Jack, 1996; Miller, et al., 1996; Cooney, et al., 1997). CERP does not eliminate cue-elicited urges, but is successful at extinguishing them. If one applies methods to attenuate potential renewal of cueelicited urges outside the treatment setting, CERP would theoretically be able to limit the probability of a relapse after treatment. In both chapters 4 and 5 , it is argued that retrieval cues reminding the patient of the treatment seting might attenuate renewal. Some support for the efficacy of such retrieval cues in clinical practice has been demonstrated by Collins and Brandon (2002) who found that both cue-elicited urge to drink and salivation can be extinguished and subsequenty renewed in social drinkers, but that when a group of these social drinkers were subtly reminded of the treatment context at test, no such renewal was found. The notion then that retrieval cues can attenuate renewal and hence increase the long term efficacy of CERP is promising, but requires future testing in a randomised clinicall trial. 
The long term efficacy of behavioural treatments of obesity is also wery poor (Lowe, 2003). Most of these treatments of obesity comprise sone combination of prem scribed restrained eating (i.e., a low calorie diet) and exercise. Although this can lead to a desirable loss in weight, this weight loss is almost never maintained. People are highlly motivated to eat what they like, and what obese persons like to eat are primarily high-caloric foods. Treatment should thus not only comprise exercise and dieting but should also try to affect the excessive appetite for especially high-caloric foods. One option might be to repeatedly expose obese persons to personalised palatable food cues at the same time preventing them to eat. Although such an exposure treatment procedure has been found to negatively affect the urge to eat (see Nederkoom, 2002; Jansen, Elgersma, \& Meijboom, 2002), conditioned taste preferences have in general been found to be difficult to extinguish. A more indirect method of affecting taste preferences then would be to learn obese persons a taste preference for more healthy, low-caloric food items. This then could facilitate adherence to a low-callorie diet and promote eating a more calorically balanced diet in the future. Learning an appetite for low caloric food items might thus be beneficial in preventing the development of an excessive appetite for high fat food items and may increase the long term efficacy of behavioural treatment programs (see also Epstein, Wing, Valoski, \& Penner, 1987).

\section{Suggestions for Further Research}

The present studies give rise to several questions that should be addressed in future research. An important research question is whether the efficacy of CERP in the treatment of addiction can indeed be improved when incorporating retrieval cues as was argued in chap ters 4 and 5 . Some support for this notion has already been found in social drinkers (Collins \& Brandon, 2002), but this research requires replication in a clinical sample of drug or alcohol dependent patients.

When considering an excessive appetite for food, it is clear that the palatability of tastes is dynamic and that the changes in food liking can be attributed to learning through experience. What then is still unclear are the factors affecting the acquisition of taste preferences. In chapter 8 it was suggested that the satiating effects, amount and rapidity of onset, determine the acquisition of a calloric conditioned taste preference. However, whether satiating potency determines or otherwise affects the reinforcing property of macronutrients in caloric conditioning awaits empirical validation.

Flawour preferences can also be acquired by pairing a neutral flavour with an already liked flavour. In accordance with Pearce (2000), it was suggested that such evaluative conditioning of flavour preferences reflects stimulus generalisation and configural 
learning, rather than Pavlovian conditioning (chapter 9). Although this model can explain most evaluative conditioning findings, it wartants empirical validation. One specific prediction of this model is that compound evaluative conditioning should lead to less transfer from the affective stimulus to the compound than when a single stimulus is paired with the same affectwe stimulus.

In sum, fundamental research is required to determine the exact role of Pavlovian learning in the development and maintenance of addictive behaviour and to investigate whether CERP can be improved in regard to preventing relapse. Further research is also needed to clarify how exactly laste preferences are learned and to determine whether the learning of taste preferences for low-caloric food items may be beneficial in increasing the long term efficacy of the treatment of obesity. 


\section{SUMMARY}

Every now and then, many people like to have a few drinks or enjoy eating an elaborate high-caloric fastfood meal. I know I do. There is not much harm in such appetitive behaviour, but when appetites become excessive this may lead to either the development of an addiction or obesity. Such an excessive appetite poses obvious health threats and additionally has been found extremely difficult to treat effectively (Orford, 2001; Lowe, 2003; McLellan, Lewis, O’Brien, \& Kleber, 2000).

One is not simply born with an excessive appetite. An appetite only becomes excessive with experience; that is experience with drugs or alcohol in the case of addiction, or experience with high-caloric food in the case of obesity. One of the most basic forms of such learning through experience is associative learning, or conditioning; the learning of a link between two specific events. The central theme of the present thesis concerned the Pavlovian learned nature of an excessive appetite, represented by studies concerning the associatively learned nature of an excessive appetite for either drugs or alcohol, or for (especially high-caloric) food. But what is Pavlovian conditioning?

Pavlov (1927) showed that dogs come to salivate to the presentation of a tone when this particular tone had always preceded food. Apparently, the dogs had learned an association between the tone and food. With the presentation of the tone, the dog would thus prepare for food intake. In other words, the dog had learned that the tone predicts food. Pavlow named the tone a conditioned stimulus, the outcome food an unconditioned stimulus, and sallivation to the conditioned stimulus a conditioned response. According to some learning theoreticians, the dog learned to recognise the conditioned stimulus as a cause of the unconditioned stimulus. Associative learning can thus be conceptualised as causal learning (Allan, 1993). Does this then imply that human causal inference is controlled by associative learning processes? If so, then it should be possible to replicate typical Pavlovian conditioning phenomena in humans when using a causal learning paradigm, and causal learning should then be based solely on trial-by-trial experience of cause-outcome contingencies. These two predictions were tested in three experiments (described in chapter 1). More specifically, it was tested whether blocking and unblocking of human causal learning can be accomplished.

What then is blocking? For example, imagine that Pavlov's dog is repeatedly exposed to a tone and light compound stimulus being paired with the presentation of a full feeding trough. The dog will not learn an association between the tone and the food in the trough when the dog has already learned an association between the light and the food. Because the light already predicts food, the tone is informationally redundant and hence the formation of a tone-food association is blocked. However, when the dog has associated the 
light with a half empty feeding trough the tone is not informationally redundant and thus no blocking, or unblocking, will occur (Kamin, 1969).

In a typical causall leaming paradigm, participants learn to link a potentiall cause (e.g., chemical substance) to a specific outcome (e.g, becoming ill). In the series of experiments described in chapter 1 , participants had to imagine themselves as being a medical researcher who has recently discovered a disease named Miltosis. This disease would be caused by exposure to certain chemical substances. Participants had to determine the causal relations between the different chemical substances and symptoms of Miltosis on the basis of fictitious patient files. Both blocking and unblocking could indeed be demonstrated in humans using this causal learning paradigm. However, in a third experiment it was also found that these effects do not depend on trial-by-trial leaming through experience. It can thus be concluded that Pavlovian conditioning is causal learning, but that human causal learning is not equal to associative learning.

Part one of this thesis then comprised studies concerning the Pavlovian nature of the development and maintenance of an excessiwe appetite for drugs or alcohol. According to the cue exposure model of addiction, with repeated drug use drug-related cues are assoclated with drug intake. For example, an alcoholic who always drinks whisky-cola will have associated the sight, smell, and taste of whisky-cola with alcohol intake. As a consequence, with exposure to these cues (e.g., sight, smell, and taste of whisky-cola) the body will prepare for alcohol intake. This is expressed as diverse psychophysiological reactions (e.g., changes in heart rate, sweat gland activity, body temperature and blood pressure) that are experienced as craving; in this example the strong urge to drink allcohol. This cueelicited urge is thus an important motivation for drug use and as such plays an important role in the maintenance of addictive behaviour.

More recentlly, several investigators proposed that psychophysiological cue reactivity does not reflect associative learning, but is a general arousal response (Carter \& Tiffany, 1999\%. This is not such a strange thought. Of course, exposure to one's favourite alcoholic drink with the explicit instruction to bold it, look and smell at it but being told not to drink from it (as is usually the case in cue exposure studies) must be pretty stressful for an alcoholic. The fact that the alcoholic will experience arousal in this situation, however, does not preclude the possibility that this arousal response is conditioned. In chapter 2 , it was investigated whether psychophysiological and subjective cue reactivity can be extinguished. Cue exposure with response prevention is a treatment intended to extinguish cue reactivity. Through repeated exposure to cues and being prevented from drug use, these cues lose their predictive value regarding drug intake and come to elicit less and less craving. This treatment though, did not appear to be very effective in extinguishing cue reactivity. As opposed to cue-elicited drug urges, psychophysiological cue reactivity did not extinguish. These results thus indicate that urges are not the subjective experience of psychophysiological cue reactivity. 
What then are urges, or craving? According to Thfany (1990), craving experienced by the recovering alcoholic when exposed to his favourite drink reflects frustration of not being able or allowed to drink, and thus does not represent a conditioned alcohol response. According to Tiffany, a history of excessive drinking or drug use will lead to the automatsation of substance use and whenever this antomatic behaviour is impeded this leads to an enormots urge. These urges are paired with the activation of higher-order cognitive processes, or energy. For example, if the alcoholic does not want to be abstinent, he will use this cognitive energy to find a way of still being able to drink alcohol. If he does want to remain abstinent, then he will use these cognitive resounces to prevent himself from automatic drinking. Craving thus demands cognitive resources, but these resources are capacity-limited. If craving indeed demands cognitive resources, cognitive performance should be attenuated when experiencing strong urges. In chapter 3 it was investigated whether cue-elicited urge to smoke has a direct effect on performance of a memory search task. No support for this hypothesis was found. Snokers experiencing strong urges to smoke do not necessarily perform worse on a memory search task than smokers who do not experience strong urges. Therefore, it is improbable that the urge to smoke demands cognitive resources.

Cue-elicited urges to smoke, drink, or use drugs can still be described as Pavlovian conditioned responding. As stated above, cue exposure with response prevention is a form of treatment designed to specifically eliminate, or extinguish these urges. Indeed, previous studies have shown that this treatment can be effective in extinguishing drug urges. However, the long-term efficacy of this treatment is as poor as any other treatment of addiction. The probability of a relapse is not affected by the extinction of drug urges. In chapter 4 it was argued that urges can be extinguished, but that this extinction does not entail the elimination of cue-elicited drug urges. Animal research has shown that extinguished conditioned responding can recover. When one regards cue-elicited urge as being a conditioned drug response, this implies that the urge to use drugs or alcohol can be extinguished but that this extinguished urge may recover, thus explaining the inefficacy of cue exposure with response prevention to affect the probability of a relapse.

In chapter 5 , it was investigated whether a renewal of extinguished conditioned responding also occurs in humans. According to contemporary learning theory, during extimction one learns to associate the conditioned stimulus with the absence of the unconditioned stimulus. This inhibitory association is context dependent. Outside the extinction context, the conditioned stimulus still predicts the unconditioned stimulus, and therefore, extinguished conditioned responding can be renewed outside the extinction treatment context (Bouton, 1993). Whether this renewal effect also occurs in humans was investigated in three experiments. A renewal effect was found in each of these three experiments and it appeared that the larger the difference between extinction context and test context the larger the renewal effect. However, not all results replicated previous animal research sug. 
gesting that the extimetion of conditioned behaviour in humans, in contrast to animals, is controlled by more higher-order cognitive processes.

The experiments described in chapter 5 underline the importance of context in learning. As learning does not take place in vacuum, it is conceivable that context not only plays an important role in the extinction of behaviour, but also in the acquisition of associations. The question how context affects the activation of alcohol-related cognitions stored in memory in humans was investigated in chapter 6. A large group of social drinkers (i.e., students) had to generate sentences with alcohol ambiguous words (e.g., bar). The first sentence that participants had to generate had to contain either the word beer or milk. It was hypothesised that the following presented alcohol ambiguous words would be interpreted as alcohol-related when the first sentence had to contain the word beer, whereas these words would be less easily regarded as alcohol-related when the first sentence had to contain the word milk. Indeed, such a priming effect was found. However, this effect was only apparent when participants had been tested in an office-like setting, as opposed when tested in a pub-like environment. In the latter setting, all alcohol ambiguous words were more likely to be interpreted as being alcohol-related, irrespective of whether the first sentence had to contain the word milk or beer. This then implies that environmental context itself can function as an alcohol-related cue capable of directly activating alcohol cognitions stored in memory.

Part two of the present thesis concerned studies investigating an excessive appetite for food. Both humans and animals eat what they like and leave food aside that they do not like. Such food preferences are anything but static. As many people can tell from their own experience, one can learn to appreciate certain flavours. In chapter 7 , individual differences in sensory-specific satiety between obese and normal-weight women were investigated. Sensory-specific satiety refers to a decrease in palatability of smell, colour and taste of a particular food eaten during a meal as compared to the palatability of a food uneaten. The function of sensory-specific satiety is twofold: (i) the decrease in palatability enhances the motivation to stop eating the food, and (ii) it enhances the motivation to eat a more varied meal. It was hypothesised that obese women should demonstrate less sensory-specific satiety than normal-weight women, thus being prone to overeating. No support for this hypothesis was found. In fact, results indicated the opposite; obese women demonstrated stronger sensory-specific satiety than the normal-weight women. It can thus be concluded that obese women are more motivated to eat a more varied diet. Other studies appear to support this conclusion. Obese persons in general do tend to eat a more varied diet (McCrory, et al., 1999). In the present obesogenic enviromment where high-fat food is plentiful and cheap, such dietary variety may lead to caloric owerconsumption.

The results from chapter 7 provide an explanation for why obese people tend to eat a more varied and potentially more high-caloric meal. However, what sensory-specific satiety cannot explain is the acquired fat preference typically demonstrated by obese persons 
(Rissanen, et al., 2002). Chapter 8 concerned research on the conditioning of such taste preferences. How does such conditioning come about? When a neutral taste is paired with a high caloric density, one will come to prefer this taste over another taste that was not paired with such a high caloric density. According to learning theoreticians, one learns an association between the taste and the energy that is provided by this taste (Fedorchak, 1997). The caloric density or energy value of food is determined by the amount and type of macronutrients in the food. Fat, for example, has the largest caloric density $(9 \mathrm{kcal} / \mathrm{g})$ of all nutrients. Apart from caloric density, nutrients also have specific postingestive effects. For example, carbohydrates and proteins have been found to be more satiating than dietary fat. According to Sclafani (1997), caloric density may thus not be the sole factor determining the acquisition of a conditioned taste preference. This hypothesis was tested in chapter 8. Female participants were required to consume yoghurt with a specific taste for breakfast for several consecutive weekdays. One taste was paired with a low-calorie yoghurt, a second taste was paired with a carbohydrate-enriched yoghurt and a third taste was paired with a fat-enriched yoghurt. The carbohydrate-rich taste and the fat-enriched taste were isocaloric (i.e, had equal caloric density). Participants exclusively developed a preference for the carbohydrate-enriched taste. Apparently, caloric density is not the only factor determining the acquisition of taste preferences.

Another way to learn a taste preference, is to pair a neutral taste with an already preferred taste (e.g., sweet or savoury tastes). Such affective learning is generally referred to as evaluative conditioning. Evaluative conditioning then refers to the transfer of affective value from a posituve (or negative) stimulus to a neutral stimulus when these stimuli are being paired. It has been suggested that this is a special form of Pavlovian conditioning. However, this view is contestied in chapter 9 . In this chapter it is argued that when two stimuli are presented together a configural representation of these stimuli is formed through which affective value generalises between the two elements of this particular configural representation. In other words, evaluative conditioning does not reflect the learning of a causal or predictive link between stimuli. Although this stimulus generalisation model can explain nearly all evaluative conditioning effects demonstrated sofar, it still awaits empirical validation.

At this point, one may wonder what the results described above mean. How, for instance, can these results provide valuable insights into how alcohol dependence and obesity can be prevented? According to the present thesis, the urge to drink alcohol and the preference for high caloric foods are learned through extensive experience with alcohol and high caloric food respectively. Therefore, to limit the probability of developing an excessive appetite, it would be wise to limit one"s intake of alcohol and high caloric food. In other words; enjoy, but neither eat nor drink too much. There are measures for determining whether one has been eating or drinking too much. One knows that one generally eats too much when one's Body Mass Index (BMI = body weight in $\mathrm{kg} /(\text { body length in } \mathrm{m})^{2}$ ) ex- 


\section{Excessive APPETHE}

ceeds a value of 25. As for drinking of too much alcoholl, like to quote Dean Martin, who once said: You 're not drunk if you can lie on the floor without holding on.

\section{Bon appent:}




\section{SAMENVATTING}

Veel mensen (althans ik) houden ervan om op zijn tijd een glaasje te drinken ev/of een uitgebreide fastfoodmaaltijd te nuttigen. Er is absoluut niks mis met dergelijk appetithef gedrag, maar wanneer de behoefte aan drank of calorierijk eten buitensportig wordt, kan dit leiden tot de ontwikkeling van een verslaving en/of overgewicht. Zowel verslaving als het hebben van ernstig overgewicht (ofwel obesitas) worden gekenmerkt door het hebben van een overmatige trek. Verslaving en obesitas vormen een overduidelijk gevaar voor de gezondheid en zijn daarnaast ook nog eens zeer moeilijk te behandelen (Orford, 2001; Lowe, 2003; McLellan, Lewis, O'Brien, \& Kleber, 2000 ).

Men wordt niet simpelweg geboren met een abnormale trek in drugs, alcohol of eten. Over het algemeen wordt werondersteld dat een abnomale trek aangeleerd is door ervaring. Een van de meest basale vormen van dergelijk leren door ervaring is associatief leren, of conditioneren; het leren wan een verband tussen twee specifieke gebeurtenissen. Het centrale thema van dit proefschrift betreft de rol van Pavloviaans conditioneren bij de ontwikkeling van een buitensporige trek; trek in het gebruiken van drugs of drank, of een abnormale trek in (met name callorierijk) eten. Maar wat is nu eigenlijk dat Pavloviaans conditioneren?

Pavlov (1927) toonde bij honden aan dat deze gingen kwijlen bij de presentatie van een toon wanneer deze toon altijd voorafging aan eten. De honden leerden klaarblijkelijk door herhaaldelijke ervaring een associatie tussen de toon en het eten. Bij de presemtatie van de toon bereidde de hond zich dus reeds voor op de inname van voer. Anders gezegd, de hond heeft geleerd dat de toon een goede voorspeller is van het voer. De toon noemde Pavlov een geconditioneerde stimulus, het voer de ongeconditioneerde stimulus en het kwijlen bij de presentatie van de geconditioneerde stimulus een geconditioneerde respons. Volgens sommige leertheoretici leert de hond de geconditioneerde stimulus herkennen als. een oorzaak van de ongeconditioneerde stimulus. Associatief leren kan men zodoende ook wel omschrijven als causaal leren (Allan, 1993). Maar betekent dit nu ook dat causale inferentie associatief, Pavloviaans conditioneren is? Zo ja, dan zouden typische conditioneringsfenomenen moeten kunnen worden gerepliceerd met een causaal lerenparadigma en zou het leren van oorzakelijke verbanden een puur op ervaring berust proces moeten zijn. Deze twee voorspellingen werden getoetst in drie experimenten (beschreven in hoolidstuk 1). Er werd onderzocht of blocking en unblocking van causaal leren kunnen worden aangetoond bij mensen.

Wat is blocking? Een voorbeeld. Stel, de hond van Pavlov wordt herhaaldelijk blootgestield aan een combinatie van een toon en een lampje dat wordt gepaard met de presentatie van een volle voerbak. De hond zal geen associatie leren tussen de toon en het 
voer wanneer deze eerder al een associatie heeft geleerd tussen alleen het lampje en het voer. Omdat het lampje dan al een goede voorspeller is wan voer, verschaft de toon geen extra informatie over het voer en zal het vormen van een toon-voer associatie worden geblokkeerd. Wanneer de hond echter het lampje heeft geassocieerd met het krijgen van een half gevulde voerbak, verschaft de toon wel degelijk "aanwullende' informatie over het woier zal er geen blocking, ofwel unblocking, optreden (Kamin, 1969).

In een typisch causaal lerenparadigma leren de proefpersonen een relatie tussen potentiële oorzaken (bijvoorbeeld een chemische stof) en een specifiek gevolg (bijwoorbeeld ziek worden). In de reeks experimenten beschreven in hoofdstuk 1 , dienden de proefpersonen zich woor te stellen dat zij een medisch onderzoeker zijn die zojuist een nieuwe ziekte heeft ontdekt: Miltose. Deze ziekte zou worden veroorzaakt door blootstelling aan chemische stoffen. De proefpersonen dienden aran de hand van fictieve patiëntendossiers te bepalen welke stoffen symptomen van deze ziekte veroorzaken. Zowel blocking als unblocking van causaal leren werden met deze taak aangetoond, maar in een derde experiment werd gevonden dat deze effecten niet afhankelijk zijn van stapsgewijs leren door erwaring. Pavloviaans conditioneren kan men beschouwen als causaal leren, maar dit betekent dus nog niet dat causaal leren ook gelijk is aan associatief leren.

Het eerste deel van dit proefschrift betrof vervolgens studies naar de rol van Pavloviaans conditioneren bij de ontwikkeling en instandhouding van een buitensporige trek in drugs of alcohol. Volgens het cue expostre-model van verslaving, zal men door herhaaldelijk druggebruik, bepaalde kenmerken (of cwes) die te maken hebben met druggebruik associëren met drugsinname. Een alcoholist bijvoorbeeld die altijd whisky-cola drinkt, zal de geur, kleur en smaak van whisky-cola associëren met alcoholinname. Als gevolg van deze associatie zal bij blootstelling aan cues (zoals de geur, kleur en smaak van whiskycola) het lichaam zich woorbereiden op de inname wan alcohol. Dit leidt tot psychofysiologische reacties (zoals veranderingen in hartslag, zweetklieractiviteit, lichaamstemperatuur en bloeddruk) die worden ervaren als drang; in dit voorbeeld een sterk verlangen om te drinken. Deze door cues onllokte drang vormt dus een belangrijke motivatie voor druggebruik en houdt zodoende het versla-vingsgedrag in stand.

Meer recentelijk werd door onderzoekers geopperd dat psychofysiologische cue reactiviteit niet het gevolg is van associatief leren, maar eerder een algemene stressreactie vormt (Carter \& Tiffany, 1999). Dit is niet zo'n heel vreemde gedachte. Het is natuurlijk behoorlijk stresserend voor een alcoholist wanneer deze een glas met zijn favoriete drank krijgt aangereikt met de instructie dit glas vast te houden en er aan te ruiken zonder er van te mogen drinken (zoals gebruikelijk is in cue exposume studies). Dat de alcoholist in dit geval stress ervaart, sluit echter niet uit dat deze stress-reactie geconditioneerd is. In hoofdstuk 2 stond de vraag centraal of psychofyiologische responsen en drang bij blootstelling aan cues kunnen worden uitgedoofd bij aan drugs of alcohol verslaafde patiënten. Cue exposure met respons-preventie is een vorm van behandeling met als doel cue reac- 
initeit wit te doven. Door herhaaldelike blootstelling aan ches, zonder dat men mag gebruiken, verliezen de cues hun voorspellende waarde en zullen deze steeds minder drang ontlokken. Uit de resultaten bleek echter dat deze behandelng niet geheel effectief is in het witdoven van cue reactiviteit. In tegenstelling tot drang, doofde de psychofysiologische cue reachiviteit niet ut. Uit deze resultaten blijt dus dat drang niet de subjectieve ervaring is van door cues ontlokte psychofysiologische reacties.

Wat is drang dan nu precies? Volgens Tiffany (1990) is de drang die de alcoholist in behandeling ervaart bij blootstelling an zijn favoriete drank een witing van zijn frustratie van het niet kunnen en mogen drinken en dus niet zozeer een geconditioneerde respons. Volgens Tiffany wordt door het vele gebruiken van drugs en/of alcohol dit verslavingsgedrag automatisch, en wanneer om wat voor reden dan ook dit automatisch gedrag wordt verhinderd, leidt dit tot enorme drang. Deze drang gaat gepaard met de mobilisatie van cognitieve energie. Wanneer de alcoholist uit het voorbeeld niet abstinent wil blijven, dan zal deze cognitieve energie worden gebruikt om een manier te vinden om toch te kunnen drinken. Wanneer de alcoholist jüst wel abstinent wil blijven, zal hij deze cognitieve energie aanwenden om te voorkomen dat hij uit automatisme weer gitat drinken. Drang vereist dus cognitieve energie, maar deze energie is niet oneindig beschikbaar. Wanneer drang cognitieve energie vereist, zou prestatie op een cognitieve tak dus moeten verslechteren wanneer men een sterke drang ervaart. In hoofdstuk 3 werd onderzocht of door cues ontlokte drang on te roken een direct effect heeft op de prestatie op een geheugentaak. Er werd geen steun voor deze hypothese gevonden. Rokers die een sterke drang ervaren presteren niet zondermeer slechter op een geheugentaak dan rokers die geen sterke drang ervaren. Het lijkt dus onwaarschijnlijk dat de drang om te roken gepaard gaat met de activatie van cognitieve processen.

De door cues ontlokte drang om te roken, drinken of drugs te gebruiken, kan dus nog steeds het best worden beschreven als zijnde een geconditioneerde respons. Zoals reeds hierboven beschreven, is cue exposure met responspreventie een vorm van behandeling dat specifiek is gericht op het uildowen van de drang on drugs te gebruiken. In eerdere studies werd reeds aangetoond dat het uitdowen van drang heel goed mogelijk is met deze behandeling. Niettemin werd ook aangetoond dat de effectiviteit van deze behandeling op de lange termijn beperkt is. De kans op terugval blijft groot na succesvolle uitdoving van drang. In hoofdstuk 4 werd betoogd dat de door cues ontlokte drang well kan worden uitgedoofd, maar dat dit niet betekent dat deze respons dan ook echt voorgoed werdwenen is. In dierexperimenteel onderzoek is herhaaldelijk aangetoond dat uitgedoofde geconditioneerde responsen kunnen terugkeren. Wanneer men door cues onlokte drang beschouwt als zijnde een geconditioneerde respons, dan impliceert dit nu dat de drang om alcohol te drinken of drugs te gebruiken wel kan worden uitgedoofd, mar dat deze uitgedoofde subjectieve cue reactiviteit ook weer kan terugkeren. Hierdoor blijft de kans op een terugval na behandeling groot. 
Hoofdstuk 5 betrof de vraag of de terugkeer van uitgedoofde geconditioneerde responsen ook optreedt bij mensen. Volgens leertheoretici leert men tijdens een uitdovingsof extinctieprocedure dat de geconditioneerde stimulus het uitblijven van de ongeconditioneerde stimulus voorspelt. Deze zogeheten inhibitoire associatie is sterk contextaflankelijk. Buiten de context waarin extinctie plaatsvond, voorspelt de geconditioneerde stimulus nog altijd de ongeconditioneerde stimulus en dus kunnen uitgedoofde geconditioneerde responsen buiten de extinctiecontext terugkeren (Bouton, 1993). Of dit nu ook bij mensen het geval is, werd onderzocht in een serie van drie experimenten. In ieder van deze experimenten werd dit effect inderdaad gevonden en daarnaast bleek dat hoe meer de extinctiecontext verschilde van de testcontext, des te sterker dit effect optrad. Niettemin bleken miet alle resultaten in overeenstemming met eerder dierexperimenteel onderzoek. Het is mogelijk dat de uitdoving van geconditioneerd gedrag bij mensen onder meer cognitieve controle staat dan bij dieren het geval is.

De experimenten uit hoofdstuk 5 onderstrepen het belang van context bij leerprocessen. Gezien het feit dat leren niet plaatsvindt in een vacuüm, is het logisch te veronderstellen dat context niet alleen belangrijk is bij het uitdoven van geconditioneerde responsen, doch ook een rol speelt bij het aanleren van associaties. In hoofdstuk 6 werd de vraag onderzocht hoe omgeving de activatie van alcohol-gerelateerde cognities bij mensen kan beïnvloeden. Een grote groep sociale drinkers (lees studenten) dienden zinnen te maken met alcoholambigue woorden (bijvoorbeeld kater). De eerste zin die de proefpersonen moesten maken was met het woord bier of melk. De hypothese was dat men de hieropvolgende alcoholambigue woorden eerder zou beschouwen als alcohol-gerelateerd, wanneer men de eerste zin met het woord bier had moeten maken, dan wanneer men de eerste zin met het woord melk had moeten maken. Dit effect werd inderdaad gevonden, doch alleen wanneer de proefpersonen werden getest in een kantooromgeving. Wanneer proefpersonen werden getest in een kroegachtige omgeving, bleken de proefpersonen ongeacht het woord voor de eerste zin de alcoholambigue woorden eerder te beschouwen als alcoholgerelateerd. Dit betekent nu dat context zelf als een alcohol cue kan fungeren en zodoende direct alcohol-gerelateerde cognities kan activeren.

Deel twee van dit proefschrift betrof onderzoek naar de buitensporige behoefte aan met name calorierijk eten. Mens en dier eten wat ze lekker vinden en laten staan wat ze vies vinden. Dergelijke uitgesproken voorkeuren voor bepaalde smaken zijn niet statisch. Zoals iedereen uit eigen ervaring wel weet, kan men bepaalde smaken leren waarderen. In hoofictstuk 7 werd een studie beschreven naar mogelijke verschillen in sensorischspecifieke verzadiging tussen obese vrouwen en vrouwen met normaal gewicht. Sensorisch-specifieke verzadiging is de afname in de smakelijkheid van de geur, kleur en smaak van bepaald voedsel tijdens een maaltijd, in vergelijking tot de smakelijkheid van voedsel dat niet tijdens deze maaltijd werd gegeten. Dit fenomeen heeft twee functies: namelijk (i) de afname in smakelijkheid bevordert de motivatie om te stoppen met eten van dit voedsel 
en (ii) het bevordert de motivatie tot het eten van een meer gevarieerde maaltijd. De hypothese werd getoetsi dat obese vrouwen minder snel en sterk dit effect vertonen en zodoende vaker bepaald voedsel overeten. Er werd geen steun voor deze hypothese gevon den. Sterker, uit de resultaten bleken de obese vrouwen juist sterkere sensorisch-specifieke verzadiging te vertonen. De conclusie luidt dat obese vrouwen sneller een bepaalde smaak beu zijn en dus meer gemotiveerd zijn om een zo gevarieerd mogelijke maaltijd te eten. Andere studies lijken deze conclusie te ondersteunen. Obese personen eten over het algemeen meer gevarieerd (McCrory, et al., 1999) en in de huidige maatschappij waar het aanbod van vette voedingsmiddelen enom is en enorm goedkoop kan dat tot een overconsumptic van calorieèn leiden.

De resultaten uit hoofdstuk 7 beschrijven hoe sensorisch-specifieke verzadiging kan verklaren dat obese personen meer gevarieerd eten. Wat dit effect niet kan verklaren is de specifieke voorkeur voor de smaak van vette voeding die obesen hebben (Rissanen, et al., 2002). Het conditioneren van een dergelijke smaakvoorkeur stond centraal in hoofdstuk 8. Wanneer een neutrale smaak wordt gekoppeld aan een hoge calorische dichtheid, dan zal men deze neutrale smaak meer gaan waarderen dan een andere smaak die niet aan een dergelijke hoge calorische dichtheid is gekoppeld. Volgens leertheoretici leert men dus een associatie tussen de smakk en de energie die deze smaak oplevert (Fedorchak, 1997). De calorische dichtheid of hoeveetheid energie van voedsel wordt bepaald door de hoeveelheid en type voedingsstof in dit voedsel. Zo heeft vet de hoogste calorische dichtheid van alle voedingsstoffen $(9 \mathrm{kcal} / \mathrm{g}$ ). Voedingsstoffen hebben echter verschillende postingestieve effecten. Zo zijn bijvoorbeeld koolhydraten en eiwitten meer verzadigend dan vetten. Volgens Sclafani (1997) zou calorische dichtheid dan ook niet de enige factor van belang zijn bij het conditioneren van een smaakpreferentie. Of dit zo is, werd onderzocht in hoofdstuk 8. In de in dit hoofdstuk beschreven studie dienden vrouwen een aantal opeenvolgende ochtenden een yoghurt-ontbijt te nuttigen: een bepaalde smaak koolhydraatrijke yoghurt, een bepaalde smaak vetrijke yogh-urt en een gegeven smaak calorie-arme, magere yoghurt. De koolhydraatrijke en de vetrijke smakk hadden een even grote calorische dichtheid, maar de proefpersonen ontwikkelden uitsluitend een voorkeur voor de koolhy. draatrijke smaak. Er kan dus worden geconcludeerd dat calorische dichtheid inderdaad niet de enige factor is die een rol speelt bij het aanleren wan een smaakpreferentic.

Een andere manier waarop een smaakpreferentie kan worden aangeleerd, is door een neutrale smaak te koppelen aan een reeds gewaardeerde smak (bijvoorbeeld zoete of zoute smaken). Deze vorm van affectief leren wordt ook wel evaluatief conditioneren genoemd. Evaluatief conditioneren is de overdracht van affectieve waarde van een reeds positief (of negatief) gewaardeerde stimulus naar een neutrale stimulus wanneer deze stimuli gecombineerd worden aangeboden. Er is gesuggereerd dat dit een speciale vorm van Pavloviaans conditioneren betreft. Dit wordt echter betwist in hoofdstuk 9 . In dit hoofdstuk wordt gesteld dat wanneer twee stimuli samen worden gepresenteerd, deze als een enkel- 
voudige configurele stimulus worden beschouwd. Generalisatie van affectieve waarde via deze configurele stimulus verklaart dan de overdracht van affectieve waarde. Dit model verklaart de meeste gevonden evaluatieve conditioneringseffecten, maar behoeft nog empirische validatie:

Aangekomen op dit punt, zult u zich misschien afvragen wat het bovenstaande nu concreet betekent. Hoe kan mu bijvoorbeeld een alcobolverslaving en obesitas worden voorkomen? In dit proefschrift wordt betoogd dat de drang om alcohol te drinken en de voorkeur voor callorierijke smaken worden geleerd door de respectievelijke ervaring met alcoholl of calorierijk voedsel. Om de kans op het ontwikkelen van zulke overmatige trek te beperken, is het dus verstandig om de inname van alcohol en calorierijk voedsel te beperken. Anders gezegd, geniet, maar eet en drink met mate. Maar wanneer heeft men nu teveel alcohol gedronken of teveel calorieên gegeten? Welnu, u weet dat u doorgaans teveel eet op het moment dat uw Body Mass Index (BMI = lichaamsgewicht in $\mathrm{kg} /\left(\right.$ lichaamslengte in $\mathrm{m}^{2}$ ) groter is dan 25 . En voor het teveel drinken van alcohol geldt, zoals Dean Martin ooit zei: You're not drunk if you can lie on the floor without holding on.

\section{Bon appetit.}




\section{REFERENCES}

Alan, L.G. (1993). Human contingency judgments: Rule based or associative? Psychologicol Bullewh, $1 / 4$. $435-448$

Arcediano, $\mathbb{F}$. Ortega, N., Matute, H. (1996). A behavioural preparation lor the study of human Pavlovian conditioning. Qwarterty Lownal of Expermenal Psychology,40B, 270-283.

Atkinson, R.C., \& Estes, W.K. (1963). Stimulus sampling theory. In R.D. Luce, R.B. Bush, \& E: Galanter (Eds.), Handboak of Mathematical Psychology (Vol 3., pp. 121-268). New York: Willy.

Baeyens, F., Crombez, G., De Houwer, J., \& Eelen, P. (1996). No evidence for modulation of evaluative havor-flavor associations in humans. Learming and Motivation, $27,200-241$.

Baeyens, F., Crombez, G., Hendrickx, H., \& Eelen, P.(1995). Parameters of huntan evaluative tavor:fawor conditioning. Leaning and Motivation, 26, 141-160.

Breyens, F., \& De Houwer, J. (1995). Evaluative conditioning is a qualitatively distinct form of classical conditioning: A reply to Davey (1994). Behaviow Research and Therapy, 33, 825 831 .

Baeyens, F., De Houwer, J., Vansteenwegen, D., \& Eelen, P. (1998). Ewaluative conditioning is a form of associative learning: On the artifactual mature of field and Davey's (1997) artifachall account of ewaluative leaming. Learning and Morivation, 29, 461-474.

Bayens, F., Eelen, P., Crombez, G., \& Van den Bergh, O. (1992). Human evaluative conditioning: Acquisition trials, presentation schedule, evaluative style, and contingency awareness. Behaviout Rowerwh and Therapy, 30, 133-142.

Baeyens, F., Eelen, P., Van den Bergh, O., \& Crombez, G. (1989). Acquired affective-waluative value: Conserwative but not unchangeable. Behaviour Research and Therapy, 27, 279-287.

Baeyens, F., Eelen, P., Van den Bergh, O., \& Crombez, G. (1990). Flavor-flawor and color-flavor conditioning in humans. Leaming and Motivation, 21,434-455.

Baeyens, $F_{.}$, Eelen, P., Van den Bergh, O., \& Crombez, G. (1992), The content of learning in human evaluative conditioning: Acquired valence is sensitive to US revaluation. Leaming and Motwation, 23, 200224.

Baeyens, F., Hendrickx, H., Crombez, G., \& Hermans, D. (1998). Neither extended sequential nor simultaneous feature positive traning result in modulation of evaluative flavor conditioning in humans. Appeaite, 31, $185-204$.

Baeyens, F., Hermans, D., \& Eelen, P. (1993). The rolle of CS-US contingency in human evaluative conditioning. Behowion Research and Therapy, 31,731-737.

Barker, C., Pistrang, N., Elliot, R. (1994). Reseanch wethods in chimicat and counthing psychology: Chichester: Wiley.

Baron, R.M., Kenny, D.A. (1986). The moderator-mediator variable distinction in sacial psychological research: Conceptual, strategic and statistical considerations. Jowral of Personaliry and Shoctal Psychology, 51, 1173-1182.

Baxter, B.W, Hinson, R.E. (2001). Is smoking automati? Demands of smoking behavior on attentional resources. Joumal of Abnomal Psychology $110,59-66$.

Beck, A.T., Beamesdorfer, A. (1974). Assessment of depression: The Depression Inventory. In P. Pinchot (Ed.), Psychological measurements in psychopharmacology (pp. 151-169). Basel: Karger.

Bell, E.A., Roe, L., S, \& Rolls, B.S. (2003). Sensiory-specific satiety is affected more by wolume than by energy content of a liquid food. Physiology \& Behawor, 78, 593\%600. 


\section{CXCESTE: APPETITE}

Birch, L.L., \& Fisher, J.A. (1996). The role of experierce in the development of children"s eating behavior. In E.D. Capaldi (Ed), why we eat what we eat: The psychology of eating (pp. 113-141). Washington, DC: American Psychological Association.

Birch, L.L., Mclphee, $L_{\text {, }}$ Steinberg, $\mathbb{L}_{\text {. }}$ \& Sullivan, $S$. (1990). Conditioned flavour preferences in young childrent. Physiology at Behavior, 47, 501-505.

Blundell, J.E., Lawton, CL, Cotton, J.R., \& Macdiarmid, L.L (1996). Control of human appetite: Implications for the intake of dietary fat. Anmual Rewiew of Numition. $16,285.319$.

Bolles, $\mathrm{RCC}_{.}$, Hayward, $\mathrm{L}_{\alpha}$ \& Crandall, $\mathrm{C}_{\text {. }}$ (1981). Conditioned taste preferences based on caloric density. Journal of Experimental Psychology: Animal Behavior Processes, 7, 59-69.

Booth, D.A., Mather, P., Fuller, J. (1982). Starch content of ordinary foods associatively conditions human appetite and satiation. Apperife, 3,163-184.

Bouton, ME. (1988). Context and aribiguity in the extinction of emotional learming: Implications for exposure therapy. Behowiour Research and Therapy, 26, 137-149.

Bouton, M.E. (1993). Context, time and memory retriewal in the interference paradigms of Pavlowian learning. Psychological Bulletin, 114, 80-99.

Bouton, M.E. (1994a). Context, ambiguity, and classical conditioning. Current Direcfions in Psychological Science, 3. 49.53.

Bouton, M.E. (1994b). Conditioning, remembering, and forgetting. Joumal of Experimemal Psychology. Animal Behavior Processes, 20, 219-231.

Bouton, M.E., \& Bolles, R.C. (1979\%. Contextual control of the extinction of conditioned fear. Leaning and Motination, 10, 445-466.

Bouton, M.E., \& Peck, C.A. (1989). Context effects on conditioning, extinction, and reinstatement in an appetitive conditioning preparation. Animal Learning and Behovior, $77,188-198$.

Bouton, M.E., \& Swarlzentruber, D. (1991). Sources of relapse after extinction in Pavlowian and instrumental learning. Clinical Prychology Review, 11, 123-140.

Branch, M.N., \& Hackenberg, T.D. (1998). Humans are animals, too: Connecting animal research to human behavior and cognition. In W. O'Donohue (Ed.), Learning and behavior therapy (pp. 15-35). Needham Heights; Allyn \& Bacon.

Brooks, D.C. (2000). Recent and remote extinction cues reduce spontaneous recovery. Quarterty Journal of Experimental Psychology, 53B, 25-58.

Capaldi, E.D. (1996). Conditioned food preferences. In E.D. Capaldi (Ed.), Why we eat what we eat: The psychology of eating (pp.53-80). Washington DC: American Psychological Association.

Carter, B.L.., \& Tiffany, S.TI. (1999). Meta-analysis of cue reactivity in addiction research. Addiction. 94, $327-340$.

Catania, A.C., \& Laties, V.G. (1999), Pavlov and Skinmer: Two lives in science Journal of the Experimental Analysis of Behavior, 72, 455-461.

Copeda-Benito, A., Tiffiny, S.T. (1996). The use of a dualatask procedure for the assessment of cognitive effort associated with cigarette craving. Psychophamacology, 127, 155-163.

Chapman, G.B. (1991). Trial order affects cue interaction in contingency judgment. Journal of Experimental Psychology: Learning. Memory, and Cognition, 17, 837-854.

Chapman, G.B., \& Robins, S.J. (1990). Cue interaction in human contingency judgment. Mentory \& Cognifion, $18,537-545$.

Cheng, P.W. \& Novick, L.R. (1992). Covariation in matural causal induction. Psychological Review, 99, 365382.

Cheng, P.W. (1997). From covariation to causation: A causal power theory. Psychological Review, 104, 367405.

Cohen, J. (1992). A power primer. Psychological Bulletin. 112. 155-159. 
Collins, B.N. \& Brandon, T.H. (2002). Effects of extinction context and retrieval cues on aloohol cue reactivity among nonalcoholic drinkers. Journal of Conswhing and Climical Psychologig, 70, 390-397,

Conklin, C.A., \& Tiffany, S.T. (2002). Applying extinction research and theory to cue-exposure addiction treatments, Addiction, 97, 155-167.

Cook, T.D., \& Campbell, D.T. (1979). Quasi-experimeintation: Design d anahgis issues for field sethongs. Chicago: Rand McNally Publishing Company.

Conney, N.L., Litt; M.D., Morse, P.A., Bauer, L.O., Gaup, L. (1997). Alcohol cue reactivity, negative mood reactivity, and relapse in treated alcoholic men. Joumal of Abnormal Psyolology, 106, 243-250.

Cramer, R.E., Weiss, R.F., William, R., Reid, S., Nieri, L., \& Manning-Ryan, B. (2002). Human agency and associative learning: Pavlovian principles govern social processes in causal relationship detection. Quarierty Joumal of Experimental Psychoiogy, 55B, 241-266.

Cunningham, C.L. (1998). Drug conditioning and drug-seeking behavior. In W. O'Donolne (Ed.). Learmg and behavior therapy (pp. 518-544). Boston: Allyn \& Bacon.

Dawson, M.E., \& Biferno, M.A. (1973). Concurrent measurement of awareness and electrodermal classical conditioning. Jownal of Experimental Pyychology, 101, 55-62.

Davey, G.C.L. (1994a). Is evaluative conditioning a qualitatively distinct form of classical conditioning? Behaviour Research and Therapy, 32, 291-299.

Davey, G.C.L. (1994b). Defining the important questions to ask about evaluative conditioning: A reply to Martin and Levey (1994). Behaviour Research and Therapy, 32, 307-310.

Dawe, S., \& Powell, J. (1995). Cue exposure treatment in opiate and cocaine dependence. 1a D.C. Drummond, S.T. Tiffany, S. Glautier, \& B. Remington (Eds.), Addictive behowour: Cre exposure theory and practice (pp. 197-209), Chichester: Willey.

Dawe, S., Powell, J., Richards, D., Gossop, M., Marks, I., Strang, J., \& Gray, J.A. (1993). Does postwithdrawal cue exposure improve outcome in opiate addiction? A controlled trial. Addiction 88 . 1233-1245.

De Houwer, J., Baeyens, F., Vansteenwegen, D., \& Eelen, P. (2000). Evalwative conditioning in the picturepicture paradigm with random assignment of conditioned stimuli to unconditioned stimuli. Journal of Experimental Psychology: Aniwal Behavior Processes, 26, 237-242.

De Houwer, J., \& Beckers, T. (2002). A review of recent developments in research and theory on human contingency learning. Quarterly Joumal of Experimental Psychology, 55B, 289-310.

De Houwer, J., \& Beckers, T. (2003). Secondary task difficuity modulates forward blocking in human contingency learning. Quarterly Journal of Experimental PSyctology, 56B, 345-357.

De Houwer, J., Beckers, T., \& Glantier, S. (2002). Ouncome and cue properties modulate blocking. Quartery" Journal of Experimental Psychology, 55, 965-985,33

De Houwer, J., Thomas, S., \& Baeyens, F. (2001). Associative learning of likes and dislikes: A review of 25 years of research on human evaluative conditioning. Psychological Bwherin, 127, 853-869.

Dickinson. A., Shanks, D.R., \& Evenden, J.L. (1984). Judgement of act-outcome contingency: The rolle of selective attribution. Quarterly Journal of Experimental Pswchology, 36A, 29-50.

Drewnowski, A., Kurth, C., Holden-Wiltse, 1., \& Saari, J. (1992). Food preferences in human obesity: Carbohydrates versus fats. Appetite, $18,207-221$.

Drummond, D.C. (2000). What does cue-reactivity have to offer clinical research? Addiction, 95 (Suppl. 2), $129-144$.

Drummond, D.C., \& Glautier, S.P. (1994). A controlled trial of cue exposure treatment in alcohol dependence. Journal of Consulting and Chinical Pychology, $62,809-817$.

Drummond, D.C., Tiffany, S.T., Glautier, S., \& Remington, B. (Eds.) (1995). Addictive behaviour: Cue exposure theory and practice. Chichester: Willey. 
Earlywine, M (1995). Expectancy accessibility, alcohol expectancies, and intentions fo consume alcohol. Journal of Applad Sochal Psychology, $25,933.943$.

Epstein, L.H. Patuch, R, \& Coleman, K.J. (1996). Differences in sallivation to repeated food cues in obese and nonobese women. Psychosomatic Nodicine, 58, 160-164.

Epstein; L.H. Radefer, J.S., Wistiewski, L.. Caggiula, A.R. (1992). Habituation and dishabiauation of human salivary responge Physiology \& Behowion $51,945-950$.

Epsterin, L.H. Wing R.R. Valoski, A., \& Penner; B.C. (1987), Stabillicy of food preferences during weight control: A sudy with 8 - to 12-year-old children and their parents. Behurior Hodification, 11, 87-101.

Fedorchak: P.M. (1997). The nature and strength of caloric conditioning. In M.E. Bouton, \& M.S. Fanselow (Eds.) Leaming. monwation and cognition: The functional behaworism of Raber C. Bolles (pp. 255. 269). Washington DC: American Psychological Association:

18jeld, A.P.; \& Dawey, G.C.L. (1997). Conceptual conditioning: Evidence for an artifactuall account of evaluative leaning. Learning and Motivation, $28,446-464$

Fiveld, A.P. B Davey, G.C.L. (1999). Reevaluating evaluative conditioning: A nonassociative explanation of conditioning effects in the wisual evaluatue conditioning paradigm. Joumal of Experimental Psychot ogy: Anirnal Behavior Processes; $25,211-224$.

Foulds, J., Stapleton, J., Feyerabend, C., Vesey, C., Jarvik, M., \& Russell, M.A.H. (1992). Effect of transdermal nicotine patches on cigarette smoking" A double blind crossover study. Psychopharmacology. $106,421-427$

Garcia, $\sqrt{ }$, \& Koelling, R.A. (1966). Relation of cue to consequence in awoidance learning. Psychonowic Science, $4,123-124$.

GarciamGutiarrez, A., \& Rosas, I.M. (2003). Enpirical and theoretical implications of additivity between reinstatement and renewal after interference in causal learning. Behavoural Processes, 63, $21-31$.

Glautier, S., \& Remington, B. (1995). The form of responses to drug cues. In D.C. Drummond, S.T. Tiftany, S. Glautier, \& B. Remington (Eds.), Addictive behaviowr: Cue exposwre theory and practice (pp. 21 46). Chichester: Wiley.

Glatier, S., S Spencer, K. (1999). Activation of alcohol-related associative neworks by recent alcohol consumption and alcohol-related ches. Addichion, 94, 1033-1041.

Gotsdanker, R., Perkins, T., Aftab, J. (1986). Studying reaction time with nonaging intervals: An effective procedure. Behavior Reseanch Methods, Lnstments, and Computers, 18, 287.292.

Greeley, J., \&yan, C. (1995). The role of interoceptive cues for drug delivery in conditioning models of dnug dependence. In D.C. Drummond, S.T. Tilfany, S. Glautier, \& B. Remington (Eds.), Addictive behavion : Cue exposwe theory and practice (pp. 119-136). Chichester: Wiley.

Gross, T.M., Jarvik, M.E., \& Rosenblat, M.R. (1993). Nicotine abstinence prodices content-specific Stroop interference. Psychopharnacology, 110, 333-336.

Grumberg, N.E., \& Singer, J.E. (1990). Biochenical measurement. In I. $T$. Cacioppo, d L G. Tassinary (Eds.) Principles of psychophysiology. Physical. social and inferential elements (pp. 149-176). New York: Combridge University Press.

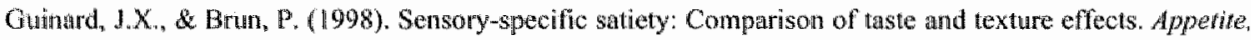
31. $141-157$

Gunther, L.M., Deniston, J.C. \& Miller, R.R. (1998). Conducting cue exposure in multiple contexis can prevent relapse. Behaviour Research and Therapy, 36, 75-91.

Hall, G., \& Iloney, R.C. ( 1990$)$. Context-specific conditioning in the conditioned-enotional-response procedure. Jommal of Experimental Pychology: Awmal Behaviom Processes, 16. 271 -278.

Hammer, M., Bloch, M., \&. Silverthorne, C.P. (1997). Effects of US alone presentations on human evaluative conditioning. Learming and Marination. 28,491-509. 


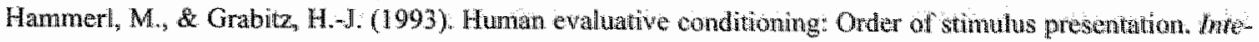
gratue Phystological and Behowional Sclance; $28,191-194$.

Hammerl, M., \& Grabit, H.J. (1996). Humam evaluative conditoning without expentencing a valued event. Learming amo Motwation, 27, 278-293.

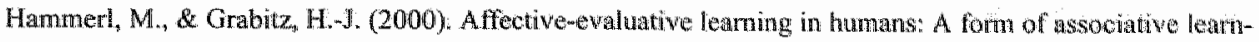
ing or only an artifact? Leawing and Motwation, $31,345-363$.

Hammersley $R$, (1992). Cue exposure and learning theory. Addichve Buhwions, $17,297-300$.

Havermans, R.C., \& Jamsen, A. (2003). Increasing the efficacy of cue exposure treatment in preventing mlapse of addictive behavior. Addictive Rehaviors, $28,989-994$.

Heather, N., \& Bradley, B.P.(1990). Cue exposure as a practical treament lor addictive disorders: Why are we waiting? Addictive Behawiors, $15,335-337$.

Heather, N., \& Robertson, 1. (1997). Problem drimking. Oxford: Oxford Medical Publicutions.

Heather, N., Stallard, A., \& Tebutt; J. (1991). Importance of substance cues in trelapse among heroin users: Comparison of two methods of investigation. Addichive Behowions, 16, $41-49$.

Heatherton, T.F., Kozliowski, L.T., Frecker, R.C., \& Fagerstrobm, K. (1991). The Fugerstrôm tesi for nicotine dependence: A revision of the Fagerstrom tolerance questionnaire. British Jound of Addiction, 86 , $1119-1127$.

Herman, C.P., \& Polivy, J. (1980). Restiained eating. In A.J. Stunkard (Ed), Obesiry (pp. 208-225). Philladephia: Sanders.

Hetherington, M., \& Rolls, B.J. (1989). Sensory-specific satiety in anorexia and bulinia nertosa . Annals of the New York Academy of Sciences, 575, 387-398.

Hetherington, M.M. (1996). Sensorywspecific satiety and its importance in meat termination. Neurosciemce d Biobehavioral Reviews, 20, 113-117.

Hetherington, M.., Rolls, B.J, \& Burley, V.J. (1989). The time course of sensory-specific satiety. Appethe, 12. $57-68$.

Hetherington, M.M., \& Rolls, B.J. (1996). Sensory-specific satiety: Theotetical frameworks and central characteristics. In E. D. Capaldi (Ed.), Why we eat what we eat: The psychology of eating (pp. 267-290). Washington, DC: APA.

Heyman, G.M. (1996). Resolving the contradictions of addiction. Behavioral and Brain Sciences, 19, 561610.

Hill, J.O., \& Peters, J.C. (1998). Enwironmental contributions to the obesity epidemic. Science, 280, 13711374.

Holland, P.C. (1983). Occasion setting in Pavlowian fenture positive discriminations. In M.L. Commons,

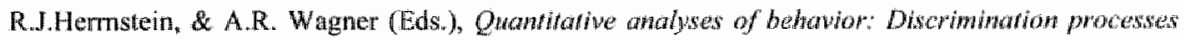
(Vol. 4, pp. 183-206). New York: Ballinger.

Humt, W.A., Barmett, L.W., \& Branch, L.G. (1971). Relapse rates in addiction programs, Jownal of Climical Psychology, 27, 455-456.

Jansen, A. (1998). A learning model of binge eating: Cue reactivity and cue exposure. Behowow Reswatch and Therapy, $36,257-272$.

Jansen, A. Elgensma, H., \& Meijboom, A. (2002). Cue exposure and cognitive therapy for bulima nainose: Are they effective? Poper presenred ar the $32^{\text {rd }}$ CABCT-Congress.

Jansen, A. \& Tenney, N. (2001). Seeing mum drinking a "light' product: Is social thearning a stronger determinant of taste preference acquisition than caloric conditioning? European Joumal of Clinical Numi tion, $55,418 \cdot 422$.

Johnson, A.K, \& Anderson, E.A. (1990). Stress and arousal. In J.T. Cacioppo, \&. L. Tassinary (wds. J, Principles of psychophysiology Physical, socral and inferenral elements (pp. 216-252). New York: Cambridge Uniwersity Press. 
Johnson, S. McPhec, L., \& Birch, L.L. (1991). Conditioned preferences: Young children prefer flavors associated with high dietary fat. Physiology \& Behavior, 50, 1245-1251.

Juliañ, L.M. \& Brandon, T.H. (1998). Reactivity to instructed smoking availability and environmental cues: Evidenee with urge and reaction time. Experimental and Clinical Psychopharmacology, 6, 45-53.

Kamin, L.J. (1969). Predictability, surprise, attention and conditioning. In B.A. Campbell, \& R. Church (Eds.), Pundshment and aversive behavior (pp. 279-296). New York: Apple-Century-Crofts.

Kassel, JD. (1997). Smoking and attention: A review and reformulation of the stimulus-filter hypothesis. Cinical Psychology Review, 17, 451-478.

Kern, D.L., McPhee, L., Fisher, J. Johnsom, S. \& Birch, L.L. (1993). The postingestive consequences of fat condition preferences for llavors associated with high dietary fat. Physiology \& Behervior, 54, 71-76.

Klajner, F., Hartman, L.M., \& Sobell, M.B. (1984). Treameni of substance abuse by relaxation training: A review of its rationale, efficacy and mechanisms. Addiche Behaviors, 9, 41 -55.

Kozlowski, L.T., Pillitteri, J.L., Sweeney, C.T., Whitfield, K. E, \& Graham, J.W. (1996). Asking questions about urges or cravings for cigarettes. Psychology of Addictive Behaviors, 10, 248-260.

Larkin, M.J.W., Aitken, M.R.F., \& Dickinson, A. (1998). Retrospective revaluation of causal judgments under positive and negative contingencies. Journal of Experimental Psychology: Leaming, Memory. amd Cogrnition, 24, 1331-1352.

Levey, A.B., \& Martin, L.. (1975). Classical conditioning of human 'evaluative' responses. Behawiotr Research and Therapy, 4, 205-207.

Lieberman, D.A. (1993). Learning: Behowion and cognition (2 ${ }^{\text {mid }}$ ed.). Pacific Grove: Brooks/Cole Publishing Company.

Lipp, O., Oughton, N., \& LeLievre, J. (2003). Evaluative learning in human Pavlowian conditioning: Extinct, but still there? Learing and Motivation, 34, $219-239$.

Lovibond, P.F. (2003). Causal beliefs and conditioned responses: Retrospective revaluation induced by experience and by instruction. Jowrol of Experimental Psychology: Learning Memony, and Cagnition, 29, $97-106$.

Lovibond, P.F., Been, S., Mitchell, C., Bouton, M.E., \& Frohardt, R. (2003). Forward and backward blocking of causal judgment is enhanced by additivity of effect magnitude. Memory \& Cognition, 31, 133-142.

Lowe, M.R. (2003). Self-regulation of energy intake in the prevention and treatment of obesity: Is it feasible? Obesity Research, 11 (Suppl.), $44-59$.

Lucas, F., \& Selatami, A. (1999a), Differential reinforcing and satiating effects of intragastric fat and carbohydrate infusions in rats. Physiology de Behavior, 66, 381-388.

Lucats, F., \& Sclathini, A. (1999b), Flavor preferences conditioned by high-fat versus high-carbohydrate diets vary as a function of session length. Physiology \& Behavior, 66, 389-395.

Mats, J.H.R., Havetmans, R.C., \& Vossen, J.H.M. (2000), Factors affecting context specificity of appetitive condinioned responding. Behaniourat Processes, 48, $149-157$.

Marlati, G.A., \& Gordon, J.R. (Eds.) (1985). Retapse prevention: Maintenarce strategies in the treatment of addicrive beharions. New York: Guilford Press.

Martin, I., \& Lewey, A.B. (1978). Esvaluative conditioning. Adwances in Behaviow Research and Therapy. 1. $57-102$.

Martin, 1., Lexey, A.B. (1994). The evaluative response: Primitive but necessary. Behaviour Research and Therapy. 32. 301-305.

Matute, H. Miller, R.R. (1998). Detecting causal relations. In W. O'Donohue (Ed.), Learning and behian ior therapy (pp, 483-497). Needham Heights; Allyn \& Bacom.

Matute, H., Pinefio, O. (1998). Stimulus competition in the absence of compound conditioning. Animal Learning and Behavion, 26, 3-14. 


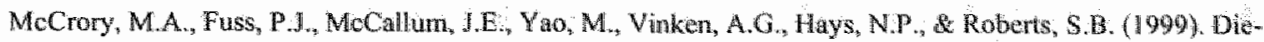
tary wariery within tood groups: Association with energy intake and body famess in men and women. Americon Joumal of Chinical Natrition. 69,440447 .

Malellan, A.T., Lewis, D.C, O'Brien, C.P., K Keber, H.D. (2000). Drug dependence, a chronic medical illness: Implications for treatment, insurance, and outcome evaluation. Journal of the Awerican Hod cal Assaciation, 284, 1689-1695.

Mehiel, R. \& Bolles, R.C. (1988). Learmed favor pretenenes based on calories are undependent of hadonto value Animal Lecaning \& Behonior; 16, 383 387.

Miller, D.L., Bell, E.A., Pelkman, CL., Peters, J.C., Rolls, B.J. (2000), Effects of dietary fat, nutrition labels, and repeated consumption on sensony-spectic saticty. Physiology d Behovior, 71, 153-158.

Millen, W.R., Westerberg, V.S., Harris, R.J., \& Tonigan, J.S. (1996). Extensions of nulapse predictors beyond high-risk situations: What predicts relapse? Prospective testing of antecedent models. Adtichon, 91 (Suppl.), 155-171.

Mowrer, R.R, \& Klein, S.B. (2001). The transituve nature of contemporary leaning theory. In R.R. Mowmer. \& S.B. Klein (Eds.), Handbook of contemporary leaming theories (pp. 1-21). Mahwah: Lawtence Erbbum Associates.

Nederkoorn, C. (2002). To binge or not to binge that she question. A critical examination of nhe conditioning model of binge eaning. Matstricht: Datawyse/Universitaite Pers Maastricht,

Nederkoorn, C., Smulders, F., Havermans, R. \& Jansen, A. (2004). Exposure to binge foad in bulimia nervosa. Finger pulse amplitude as a potential measure of urge to eat and predictor of tood intake. Appevite, 42, 125-130.

Niaura, R. Abrans, D.B., Shadel, W.G., Rohsenow, D.J., Monti, P.M., \& Sirota, A.D. (1999). Cue axposure treatment for smoking relmpse prevention: A controlled dinicall trial. Addicton, 94, 685-695.

Niemi, P., Näatänen, R. (1981). Foreperiod and simple reaction time. Psychological Bulletim, 89, $133-162$

O"Brien, C.P., Childress, A.R., MoClellan, T., E Ehman, R. (1990). Integrating systematie cue exposure with standard treatment in liecovering drug dependent patients. Adactive Behaviors, 15, 355-365.

O"Donohue, W. (1998), Conditioning and third-generation behavior therapy. In W. O'Donolwe (Ed.), Learning and Behavior Therapy (pp.1-14). Needham Heights: Allyn \& Bacon.

Orford, 1. (2001). Addiction as excessive appetite. Addiction, 96, 15-31.

Paviov, I.P. (1927). Condinioned Reflexes Oxford: Oxford University Press.

Pearce, J.M. (1987). A model for stimulus generalization in Pavlovian conditioning. Psychological Review. 94. 61.73 .

Pearce, I.M. (1994). Sunilarity and discrimination: A selective review and a comnectomist model. Psychow logical Revew, 101, 587-607.

Pearce. J.M. (2002). Evaluation and development of a contectionist dheory of configurail leaming. Amimal Learning \& Behavior. 30.73 .95$.

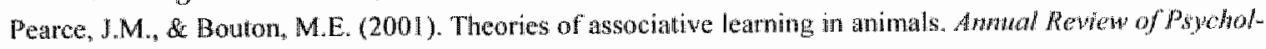
$o x y_{n}, 52,111-139$

Pérez, C. Lucas, F., \& Sclafani, A. (1995). Carbohydrate, fat, and protein condition similar flavor preferences in rats using an oral-delay procedure. Physiology \& Behovior, 57, 549-554.

Pineño, O. \& Matule, H. (2000). Interference in human predictive learning when alsociations share an conmon element. Iritertational Jowmal of Comparative Psychology. 13, 16-33.

Price, P.C., \& Yates, J.F. (1995). Associative and rute-hased accounts of cue interuction in contingency judg. ment. Joumat of Experinental Psychology. Leawing, Mewory, and Cogwnion, 21, 1639-1655.

Rah, S.K., Reber, A. S., \& Hsiao, A. T. (2000). Another wrinkle on the duallask SRT experinent: In's probably not dual task. Psychonomic Bulletin of Review, $7,309-313$. 
Ramdieh, A., \& Lolordo, V.M. (1979). Associative and nonassociative theories of the UCS prexposure phenomenon: Implications for Pavlovaan conditioning. Psychological Bulletim, 86. $523-548$.

Rankin, H. Hodgson, R., \& Stokwell, T. (1983). Cue exposure and response prevention whth alcoholics: $A$ controlled trial, Behaviour Research and Therapy, 21, 435 -446.

Ratcliff, $\mathbb{R}$ (1993), Methods for dealing with reaction time outliers. Psychological Bulletiw, 114, 510-532.

Raynor, H.A. \& Epstein, L. H. (2001). Dietary variety, energy regulation, and obesity. Psychological Bulde$\mathrm{fim}_{i} / 27,325,341$.

Rescorla, R.A. (1985). Conditioned inhibition and facilitation. In R.R. Miller \& N.E. Spear (Eds.), Informalion processing in animals: Conditioned inhibition (pp. 299-236). Hillsdale: Lawrence Erbaum Associates.

Rescorla, R.A. (1988). Pavlovian conditioning: It's not what you think it is American Psychologist, 43, 151160.

Rescorla, R.A. (2001). Experimental extinction. In R.R. Mowrer, \& S.B. Klein (Eds.), Handbook of contewyporary learning theories (pp. 1 19-154). Mahwah, NJ: Lawrence Erlbaum Associates.

Rescorla, R.A., Durlach, P.I. (1981). Within-event learning in Pavlovian conditioning. In N.E. Spear \& R.R. Miller (Eds.), Information processing in animals: Mermory mechanisms (pp. 81-1】2). Hillsdale: Lawrence Erlbaum Associates.

Rescorla, R.A. \& Wagner, A.R. (1972). A theory of Pavlowian conditioning: Variations in the effectiveness of reinforcement and nonreinforcement. In A. Black, \& W.F. Prokasy (Eds.), Classical Conditioning if (pp.64-99). New York: Apple-Century-Crofts.

Rissanen, A., Hakală, P., Lissner, L., Mattllar, C-E., Koskunvuo, M., \& Rönnemaa, T. (2002). Acquired preference especially for dietary fat and obesity: A study of weight-discordant monozygotic twin pairs. International Journal of Obesity, 26, 973-977.

Robinson, T.E., \& Berridge, K.C. (1993). The neural basis of drug craving: An incentive-sensitization theory of addiction. Brain Research Reviews, 18, 247-291.

Robinson, T.E., \& Berridge, K.C. (2000). The psychology and netrobiology of addiction: An incentivesensitization view. Addiction, 95 (Suppl. 2), 91-117.

Roehrich, L., \& Goldman, M.S. (1995). Implicit priming of alcohol expectancy memory processes and subsequent drinking bethavior. Experimental and Clinical Psychopharmacology. 3, 402-410.

Rohsenow, D.I., Monti, P.M., \& Abrams, D.B. (1995). Cue exposure treatment for alcohol dependence. In D.C. Drummond, S.T. Tiffany, S. Glantier, \& B. Remington (Eds.), Addictive behawiour: Cue exposure theory and practice. Chichester: Wiley.

Rohisenow, D.J., Monti, P.M." Rubonis, A.W., Gulliver, S.B., Colby, S.M., Binkolf, J.A., \& Abrams, D.B. (2001). Cue exposure with coping skills training and communication skills training for alcohol dependence: 6 and 12-month outcomes. Addiction, 96, 1161-1174.

Rolls, B.I. (1986). Sensory-specific satiety. Nurrition Reviews, 44: 93-101.

Rolls, B.I., \& Bell, E.A. (1999). Intake of fat and carbohydrate: Role of energy density. European Journal of Clinical Nutrition, 53 (Suppl. 1), 166-173.

Rolls, B.J., van Duijwenvoorde, P.M., \& Rolls, E.T. (1984). Pleasanthess changes and food intake in a varied four-course meal. Appetise, $5,337-348$.

Rolls, E.T., \& Rolls, J.H. (1997), Olfactory sensory-specific satiety in humans, Physiology \& Behovior, 61. $461-473$.

Rozin, P., Wrzesniewski, A., Byrnes, D. (1998). The elusiveness of evaluative conditioning. Learning and Motivation, 29, 397-415.

Rusted, J. M., Grimpiner, L., Temnant, A., \& Warburton, D. M. (1998). Effortful processing is a requirement for nicotine-induced improvements in memory. Psychopharmacology, 138, 362-368. 
Sayette, M.A. \& Hufford, M.R. (1994). Effects of cue exposure and deprivation on cognitive resources in smokers. Jawmal of Abnomal Psychology: 103, 812-818.

Sayette, M.A., Monti, P. M., Rohsenow, D. J., Gulliver, S. B., Colby, S. M., Sirota, A. D., Niaura, R., \& Abrams, D. B. (1994). The effects of cue exposure on reaction time in male alcoholics. Journal of Studies on Alcohol, 55, 629-633.

Sayette, M.A., Shiffinan, S., Tiffany, S.T., Niaura, R.S., Martin, C.S., \& Shadel, W. G. (2000). The meastrement of drug craving. Addiction, 95 (Suppl. 2), 189-210.

Schulze, D., \& Jones, B.T. (2000). Desire for alcohol and outcome expectancies as measures of alcohol cuereactivity in social drinkers. Addiction, 95, 1015-1020.

Sclafani, A. (1990). Nutritionally based learned flavor preferences in rats. In E.D. Capald (Eds.), Tarte, experience and feeding (pp. 139-156). Washington DC: American Psychological Association.

Selafani, A. (1997). Learned controls of ingestive behaviour. Apperite, 29, 153-158.

Shanks, D. R. (1985). Forward and backward blocking in human contingency judgement. Quarterly foumol of Experimental Psychology, 37B, 1-21.

Shanks, D. R. (1991). On similarities between causal judgment in experienced and described situations. Psy chological Science, 2, 341-350.

Shanks, D.R., Charles, D., Darby, R.J., \& Azmi, A. (1998), Configural processes in human associative learn ing. Journal of Experimental Pychology: Learning, Memoly and Cognition, 24, 1353-1378.

Shanks, D.R., Darby, R.J., \& Charles, D. (1998). Resistance to interference in hurnan associative learning: Evidence of configural conditioning. Joumal of Experimental Psythology: Animal Behowor Processes, 24, 136-150.

Sher, K.J. (1985). Subjective effects of alcohol: The influence of setting and individual differences in alcohol expectancies. Journal of Studies on Alcohol, 46, 137-146.

Siegel, S. (1983). Classical conditioning and drug effect, In R. Smart, F. Glaser, Y. Israel, H. Kalant, R. Popham, \& W. Schmidt (Eds.). Research adrances in alcahol and drug problens (pp.207-246). New York: Plenum Press.

Siegel, S., \& Ramos, B.M.C. (2002). Applying laboratory research : Drug anticipation and the treatment of drug addiction. Experimental and Clinical Psychopharmacology, 10, 162-183.

Spilich, G. J., June, L., \& Renner, J. (1992). Cigarette smoking and cognitive performance. Britisht Jow Addiction, 87, 1313-1326.

Stacy, A.W. (1997). Memory activation and expectancy as prospective predictors of alcohol and marihuana use. Journal of Abnormal Pychology: 106, 61.73\%.

Stacy, A.W., Leigh, B.C., \& Weingardt, K.R. (1994). Monory accessibility and association of alcohol use and its positive outcomes. Experianental and Clinical Psychopharmacology. 2. 269-282.

Stacy, A.W., Leigh, B.C. \& Weingard, K.R. (1997). An individual difference perspective applied to word association. Personality and Social Psychology Bulletin, 23, $229-237$.

Sternberg, S. (1966). High-speed scanning in human memory. Science, 193, 652-654.

Sternberg, S. (1975). Memory scanning: New findings and current controversies. Quasterdy Journat of Exw perimental Psychology, 27A, 1.32.

Stevenson, R.J., Boakes, R.A., \& Prescon, J. (1998). Changes in flavor sweetness resulting from implicil learning of a simultaneous flavorosweetness association: An example of learned synesthesia. Learning and Morivation, 29, 113-132.

Stevenson, R.J., Boakes, R.A., \& Wilson, J.P. (2000). Resistance to extinction of conditioned odor perceptions: Evaluative conditioning is not unique. Jownal of Experimenal Psychology: Leaming. Memory and Cogrition, 26, 423-440. 
Stevenson, KJ., Prescot, J., \& Boakes, R.A. (1995). The acquisition of taste properties by flavors. Learwiwg and Wotivation, 20,433455 .

Stuart, E.W. Shimp. T.A. Engle, R.W. (1987). Classical conditioning of consumer atrindes: Four experiments in an advertising context. Joumal of Consumer Research, 14, 334-349.

Stunkard, A.J. \& Penick, S.8. (1979). Behavior modification in the treatinent of obesity: The problem of maintaining weight loss. Archier of General Psychatry, 36, 801-806.

Swin, G.E., Ward, M.M., Jack, L.M. (1996). Abstinence effects as predietors of 28-day relapse in smokers. Adlfichlive Behaviors. 21, 481-490.

Swithers, S.E., Hall, W.O. (1994). Does oral experience terminate ingestion? Appetite, 23, 113-138.

Tait, R, Martin-fverson, M., Michie, P.T., \& Dusci, L. (2000). The effects of cigaret consumption on the Sternberg visual memory search paradigm. Addiction. 95, 437-446.

Tepper, B.J. (1992). Dietary restrain and responsiveness to sensory-based cues as measured by cephalic phase salivation and sensory specific satiety. Physiology \& Behavior, 52, 305-311.

Thomas, B.L., Larsen, N., \& Ayres, J.J.B. (2003). Role of context similarity in ABA, ABC, and AAB renewal paradigms: Implications for theories of renewal and for treating human phobias. Learning and Marivarion, 34, 410-436.

Tiffany, S.T. (1990). A cognitive model of urges and drug-use behaviour: Role of automatic and nonautonatic processes. Psychological Review, 97, 147-168.

Tiffany, S.T. (1997). New perspectives on the measurement, manipulation and meaning of drug craving. Human Psychopharnacology. 12 (Suppl.), 103-113.

Tiffany, S.T., Cox, L.S., \& Elash, C.A. (2000). Effects of transdermal nicotine patches on abstinence-induced and cue-elicited craving in cigarette smokers. Jowrnat of Consulting and Clinical Pyychology, 68, 233240 .

Todrank, J, Byrnes, D., Wrzesniewski, A., \& Rozin, P. (1995). Odors can change preferences for people in photographs: $A$ cross-modal evaluative conditioning study with olfactory USs and visual CSs. Learning and Motivation, 26, 116 140.

Van Hamme, L. $J_{1,}$ \& Wasserman, E.A. (1993). Cue competition in causality judgment: The role of manner of information presentation. Bulletin of the Psychonomic Society, 31, 457-460.

Van Hamme, L.J., \& Wassernan, E.A. (1994). Cue competition in causality judgments: The role of nonpresentation of compound stimulus elements. Learning and Motrivation, 25, 127-151.

Wadden, T.A., Brownell, K.D., \& Foster, G.D. (2002). Obesity: Responding to the global epidemic. Jownal of Consurung and Chricai psychology. $70,510-525$.

Waldmann, M.R. (2000). Competition among causes but not effects in predictive and diagnostic learning. Journal of Experimental Psychology: Learning, Mewory, and Cognition, 26, 53-76.

Waldmann, M.R. \& Holyoak, K.J. (1992). Predictive and diagnostic tearning within causal models: Asymmetries in cue competition. Jownal of Experimental Psychology: General, 121, 222-236.

Wall, A., McKee, S.A., \& Hinson, R.E. (2000). Assessing variation in alcohol outcome expectancies across environmental context: An examination of the situational-specificity hypothesis. Psychology of Addicthe Behations, 14, 367-375.

Wall, A. Mckwe, S.A., Hinson, R.E., Goldstein, A. (2001). Examining alcolhol outcome expectancies in laboratory and nuturalistic bar settings: A within-subject experimental analysis. Psychology of Addicine Behaviors, 15, 219-226.

Warburton, D. M., Mancuso, G. (1998). Evaluation of the information processing and mood effects of a transdermal nicotine patch. Psychopharmacology, $135,305-310$.

Waters, A. J. \& Feyerabend, C. (2000). Determinants and effects of attentional bias in smokers, Psychology of Addictive Behowiors. 14, 111-120. 
Werte, J.M. \& Sayetre, M.A. (2001) A revicw of the effects of perceived drug use opportunity on selfreported urge. Experinental and Clinical Psychopharmatology, 9, 3-13.

Wiers, R.W., Havemans, R., \& Granzier, I. (2004). Context effects on implicit and explicit alcohol-related

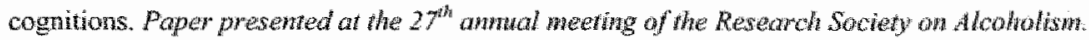

Wiers, R.W., Stacy, A.W., Ames, S.L., Noll, J.A., Sayette, M.A., Zack, M., E Krank, M. (2002). Inplicit and explicit alcoholwelated cognitions. Alcoholism: Climical and Experimental Researh, 26. 129-137.

Wikler, A. (1948). Recent progress in research on the neuroplyysiological basis of motphine addiction. American Joumal of Psychiatry, 105, 329-338.

Wise, RA., \& Bozarth. M.A. (1987). A psychomotor stimulant theory of addiction. Pyochological Revien. 94, 469-492.

Wisniewski, L., Epstein, L.H., \& Caggiula, A.R. (1992). Effect of food change on consumption, hedonics, and salivation. Physiology Behavior, 52, 21-26.

Yerkes, R.M., \& Morgulis, S. (1909). The method of Pawlow in animal psychology. Psychological Butleth. 6. $257-273$.

Zellner, D.A., Rozin, P., Aron, M., Kulish, C. (1983). Conditioned enlancement of hutnan's liking for flavors by pairing with sweetness. Learning and Motivation 14, 338-350. 
Excessrve Appettre 


\section{DANKWOORD}

Ik denk en werk graag zelfstandig. Dit betekent dat ik te valk te koppig ben om hulp te zoeken of te aanvaarden. Dergelijke koppigheid is vanzelfsprekend geen goede eigenschap en leidde er bijwoorbeeld toe dat ik nog niet zo lang geleden bij het plaatsen wan een dubbele lichtschakelaar (hoe moeilijk kan dat zim?) tot tweemaal toe $220 \mathrm{~V}$ kreeg te verduren. Niettemin, ik leef nog en de schakelaar werkt inmiddels perfect, zij het dat met de linkerknop nu de rechterlamp aan/uit gaat en met de rechterknop de linkerlamp aan/uit gaat.

Goed onderzoek is het produkt van velen. Bij het bedenken, plannen en uitvoeren van studies, heeft de onderzoeker specifieke kennis, ervaring en feedback nodig van anderen. Dat geldt ook voor mij en ik heb gedurende mijn AiO-schap dan ook moeten leren om hulp te vragen en te accepteren. Ik heb het geluk gehad dat ik hierbij hulp kreeg van de beste begeleider, promotor en baas die een AiO zich maar kan wensen: Anita Jansen. Beste Anita, ik wil je hartelijk danken voor je fantastische begeleiding en voor je geweldige vertrouwen in me.

Naast Anita had ik tal van hulpjes die ik hier eveneens wil bedanken voor hun commentaar, kritiek, discussies, heldere inzichten en assistentie bij het uitvoeren van de studies. In het bijzonder: Linda Vancleef, Saskia Debaere, Fren Smulders, Reinout Wiers, Marcel van den Hout, Sandra Mulkens, Sjaan Nederkoorm, Maarten Vanwingh, Rolf Sanderson, Anja Meijboom, Linda Kroon, Ingmar Franken, Henk Nijman, DE therapeuten van de Mondriaan Zorggroep divisie verslavingszorg, Maartje Zandbergen, Mark Litt, Roy Thewissen, Jantien Keuker, Tineke Lataster, Cody Brooks, Helena Matute, Mark Bouton, Pauline Dibbets, Bill Roberts, Eva Bylois, Leen Hermans, Anne Roefs, Katrijn Houben, John Pearce, Frank Baeyens, Ron Hellenbrand, de dunne en dikke eetgroep, EAR, Nick \& Gerard, en natuurlijk Nicole en Truus.

Wetenschap is belangrijk werk, doch vreugdeloos zonder collega's met wie je een tas koffie, een roddel en een intrusie kunt delen. Beste Eric, Sjaan, Ingrid en Horzy, jullie zorg(d)en er hier in Maastricht woor dat tussen de wetenschap door ook nog wat te lachen valt. Ontzettend bedankt hiervoor. Sjaan en Ingrid wil ik hier nog extra bedanken voor hum aanvaarding van het paranimfschap. Ik ben vereerd dat twee zulke knappe koppen mij bij willen staan. Mijn mede-AiO's dank ik hier tevens voor de koffiepauzes, de borrels en de pingpongkampioenschappen.

Tot slot wil ik pa, ma, zusje, opa, oma, Dinie, Nijs, Millie, Loek, en Wanda bedanken voor het meedenken, meevoelen en meeleven. Maar bovenal wil ik Inge bedanken...voor alles. 


\section{CURRICUlum VitaE}

Remco Comelis Havermans werd op 13 oktober 1974 geboren in Eindhoven. In 1994 behaalde hij zijn VWO-diploma aan de Gemeentelijke Scholengemeenschap Woensel (thans Stedelijk (College) te Eindhoven. In 1994 starte hij met de studie psychologie aan de Katholieke Universiteit Nijmegen (thans Radboud Universiteit Nijmegen). Na aen jaar lang psychologie te hebben verruild voor cognitiewetenschappen, vervolgde hij in 1997 zijn studie psychologie en studeerde hij in 1999 af in de richting Vergelijkende \& Fysiologische Psychologie (thans Biologische Psychologie). Nog in datzelfde jaar werd hij aangesteld als Assistent in Opleiding/Docent (AiODo) bij de capaciteitsgroep Experimentele Psychologie van de Universiteit Maastricht. Het onderzoek beschreven in dit proefschrift werd gedurende deze aanstelling uitgevoerd. Per 1 januari 2005 geniet hij een tijdelijke aanstelling als onderzoeker/docent bij de capaciteitsgroep Experimentele Psychologie. 


\section{PUBLICATIES}

Havermans, R.C. (2000). De onbeheersbare drang. Bespreking van "Behandelingsstrategieon bij Ziekelijke" Impulsen” wan C.A.L. Hoogduin \& C.P.F van der Staak (Red.). Diagnostiek-Wijzer, 4, 177-179.

Havermans, R.C. (2002). Verslaving, drang, reactietijd en rokende proefleiders. De Psycholoog 37, 3-8.

Havermans, R.C. (2003). Mensen zijn dieren, of net? Bespreking van academisch proefschrift "Humans as an animal model? Studies on cue interaction, occasion setting and context dependency' wan P. Dibhets. De Psycholoog, 38, 659-660.

Havermans, R.C., Debaere, S., Smulders, F.T.Y., Wiers, R.W., \& Jansen, A. (2001). The urge to smoke and cognitive performance (ABSTRACT). Alcoholism; Clinical and Experimental Research, 25.98A.

Havermans, R.C, Debaere, S., Smulders, F. T.Y., Wiers, R.W., \& Jansen, A. (2003). Effect of cue exposure, urge to smoke and nicotine deprivation on cognitive performance in smokers. Psychology of Addicrive Behawions, 17, 336-339.

Havermans, R.C., Hermans, L., Roefs, A., Nederkoorn., C. \& Jansen, A. (2004). Obese women denonstrane stronger sensary-specific satiety than normal-weight women. Manuscript submitted for publication.

Havermans, R.C., \& Jansen, A. (2001). Waarom cue exposure met responspreventie in de klinische setting niet effectief is De Psycholoog, 36, 234-239.

Havermans, R.C., \& Jansen, A. (2002), The efficacy of cue exposure therapy: Room for improvement (ABSTRACT)? In Abstracis 32nd EABCT Congress (p. 223). Maastricht.

Havermans, R.C., \& Jansen, A. (2003). Increasing the efficacy of cue exposure treatment in preventing telapse of addictive behavior. Addicfive Behoviors, 28, 989-994.

Havermans, R.C., \& Jansen, A. (2004). Stimulus geveralization and configural learning as a model of evaluative conditioning. Manuseript submitted for publication.

Havermans, R.C., Keuker, J., Lataster, T., \& Jansen, A. (in press). Contextual control of extinguished conditioned performance in humans. Learning and Motivation.

Havermans, R.C., Lataster, T., Thewissen, R., \& Jansen, A. (2004). Uitdoven is geen afleren: implicaties voor cue exposure therapie. Gedragstherapie, 37, 77-86.

Havermans, R.C., Mulkens, S., Franken, I., Vanwingh, M., Sanderson, R. \& Jansen, A. (2003). Cue exposure met responspreventie bij de behandeling van verslaving. Directieve Therapie, 23, 247-261.

Havermans, R.C., Mulkens, S., Nederkoom, C., Je Jansen, A. (2004). The efficacy of cue exposure with response prevention in extinguishing arug and alcohol che reachivity. Manuscript submitted for publicaltion.

Havermans, R.C., Roefs, A., Lataster, T., Houben, K., \& Jansen, A. (2003). Comparing carbohydutates and dietary far in conditioning flavowr preferences in hwhans. Manuscript submitted for publication.

Havermans, R.C., Vancleef, L., \& Jansen, A. (2004). Blocking and unblocking of cousal jwdgment: The role of oufcome magnifude in human causal learning. Manuscript submitted for publication.

Havermans, R.C, Vancleef, IL, Bylois, E., \& Jansen, A. (2004). Contex" specifac actiwation of alcoholrelated cognitions (ABSTRAC1). Alcoholsm: Clinical and Experimental Research, 28, 102A.

Havermans, R.C., Vancleef, L., Bylois, E., Wiers, R.W., \& Jansen, A. (in press). Context dependent access to alcohol-related concepts stored in memory. Alcohol Research.

Havermans, R.C., Vancleef, L., Bylois, E., \& Jansen. A. (2004). Denken aam drank: de rol wan drinkomgeving en drinkervaring. Gedrag \& Gezondheid. 32.175-181.

Huijts, A., Jurgens, T., Havermans, R., \& Martijn, C. (2003). Opgeven of doorganan: de invloed van schemaactivatie op ego-depletion. In E. wan Dijk, M. Hagendoom, \& D. Wigiboldus (Eds.) p. 15/-159, Jaurboek Sociale Psychologie. Delft: Eburon. 
Maes, J.HR, Havermans, R.C., \& Vossen, J.M.H. (1999a). Acute amphetamine administration does not affeet context degendency of appetitive conditioning (ABSTRACT). In Proceedings of the 19th Low Countries Meeting (p.34). Nijmegen.

Maes, J.H.R., Havermans, R.C., \& Vossen, J.M.H. (1999b). Factors affecting contexi specificity of appetitive conditioning (ABSTRACT). In Proceedimgs of the 19th Low Couthiries Meeting (p.35). Nijmegen.

Maes, I.H.R, Havernans, R.C., \& Vossen, J.M.H. (2000). Factors affecting context specificity of appetitive conditioned responding. Behaviow al Processes, 48, 149-157.

Martijin, C., Alberts, H.S.M., Merckelbach, H., Havermans, R., \& de Vries, N.K. (2004). If he can do it. then I can too. The influence of a rolemodel on self-control and ego depletion. Manuscript subritted for publication.

Nederkoorn, C., Smulders, F., Havermans, R., \& Jansen, A. (2004). Exposure to binge food in bulimia nervosa: Finger pulse amplitude as a potential measure of urge to eat and predictor of food intake. Appewite, 42, $125-130$.

Nederkoorn, C., Smilders, F.T.Y., Havermans, R.C., Roefs, A., \& Jansen, A. (2005). Impudsivity in obese women. Obese women controt their thoughts, bu wor their acts. Manuseript subutitted for publication.

Roefs, A., Quadackers, L., Werrij, M.Q., Wolters, G., Havermants, R. Nederkoorn, C., wan Breukelen, G., \& dansen A. (2004). The emvironment determimes whether chocolate is palatable or unhealthy. Manuscript submitted for publication.

Thewissen, R., Van den Hout, M., Havernans, R.C., \& Jansen, A. (in press). Context-dependency of cueelicited urge to smoke. Addiction.

Thewissen, R., Snijders, J.B.D., Van den Hout, M., Havermans, R.C., \& Jansen, A. (2004). Renewal of ckeelicited turge to smoke. Manuscript submitted for publication.

Wiers, R.W., De Jong, P., Havermans, R., \& Jelicic, M. (2004). How to change implicit drug-related cognitions in prevention: A transdisciplinary integration of föndings from experimental psychopathology, sociall cognition, memory and experimental learning psychology. Substance Use and Misuse 39. 1625-1684.

Wiers, R.W., Hawermans, R., \& Granzier, J. (2004). Contexi effects on the accessibility of implicit and explicit alcohol-related cognitions (ABSTRACT). Alcoholism: Clinical and Experimental Reseorch, 28, $187 \mathrm{~A}$. 